ADA 325438

Fint

i1 11

US Army Corps

of Engineers

Construction Engineering

Research Laboratories

\title{
Diffraction of Sound by Objects in the One-wavelength Size Range
}

by

Soon Young Lee

\section{For Reference}

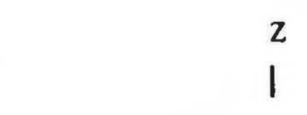

Not to be taken from this room

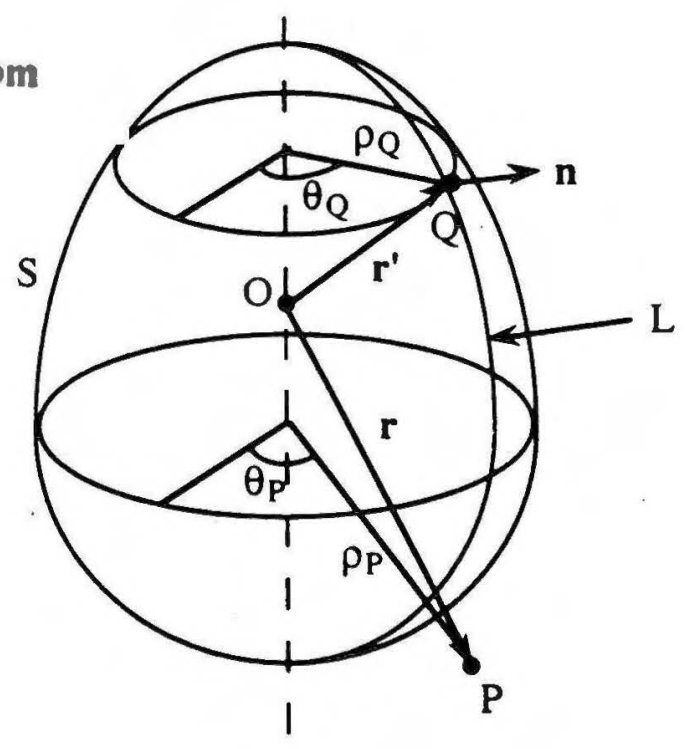

The diffraction of sound by hard geometrical shapes has been studied by the Method of Moments (MOM), a boundary element technique for solving the scalar Helmholtz integral equation. The scattered fields of several rectilinear-shaped objects are analyzed. The problem of scattering by an axially-symmetric obstacle has been simplified by using the symmetry to reduce the computational load. The validity of the resulting procedure has been verified by comparing the MoM results for a flat circular disk illuminated by either a plane or spherical wave with the results obtained by the classical method of separation of variables and expansion in oblate spheroidal wave functions. 
The contents of this report are not to be used for advertising, publication, or promotional purposes. Citation of trade names does not constitute an official endorsement or approval of the use of such commercial products. The findings of this report are not to be construed as an official Department of the Army position, unless so designated by other authorized documents. 


\section{USER EVALUATION OF REPORT}

REFERENCE: USACERL Technical Manuscript 97/73, Diffraction of Sound by Objects in the Onewavelength Size Range

Please take a few minutes to answer the questions below, tear out this sheet, and return it to USACERL. As user of this report, your customer comments will provide USACERL with information essential for improving future reports.

1. Does this report satisfy a need? (Comment on purpose, related project, or other area of interest for which report will be used.)

2. How, specifically, is the report being used? (Information source, design data or procedure, management procedure, source of ideas, etc.)

3. Has the information in this report led to any quantitative savings as far as manhours/contract dollars saved, operating costs avoided, efficiencies achieved, etc.? If so, please elaborate.

4. What is your evaluation of this report in the following areas?

a. Presentation:

b. Completeness:

c. Easy to Understand:

d. Easy to Implement:

e. Adequate Reference Material:

f. Relates to Area of Interest:

g. Did the report meet your expectations?

h. Does the report raise unanswered questions? 
i. General Comments. (Indicate what you think should be changed to make this report and future reports of this type more responsive to your needs, more usable, improve readability, etc.)

5. If you would like to be contacted by the personnel who prepared this report to raise specific questions or discuss the topic, please fill in the following information.

Name:

Telephone Number:

Organization Address:

6. Please mail the completed form to:

Department of the Army CONSTRUCTION ENGINEERING RESEARCH LABORATORIES

ATTN: CECER-TR-I

P.O. Box 9005

Champaign, IL 61826-9005 


\section{REPORT DOCUMENTATION PAGE}

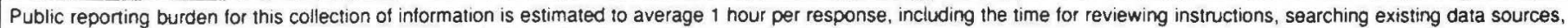

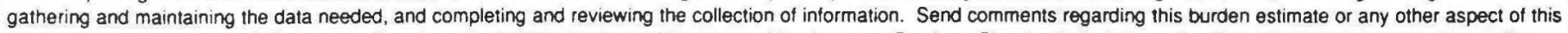

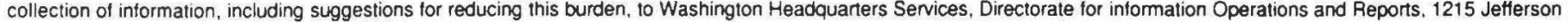
Davis Highway, Suite 1204. Arlington, VA 22202-4302. and to the Oftice of Management and Budget. Paperwork Reduction Project (0704-0188), Washington. DC 20503.

\begin{tabular}{|l|c|c|}
\hline 1. AGENCY USE ONLY (Leave Blank) & $\begin{array}{c}\text { 2. REPORT DATE } \\
\text { MaY } 1997\end{array}$ & $\begin{array}{c}\text { 3. REPORT TYPE AND DATES COVERED } \\
\text { Final }\end{array}$ \\
\hline
\end{tabular}

4. TITLE AND SUBTITLE

Diffraction of Sound by Objects in the One-wavelength Size Range

BT25

IE6

6. AUTHOR(S)

Soon Young Lee

7. PERFORMING ORGANIZATION NAME(S) AND ADDRESS(ES)

U.S. Army Construction Engineering Research Laboratories (USACERL)

P.O. Box 9005

Champaign. IL 61826-9005

8. PERFORMING ORGANIZATION

REPORT NUMBER

TM $97 / 73$

9. SPONSORING MONITORING AGENCY NAME(S) AND ADDRESSIES

US Army Center for Health Promotion and Preventive Medicine

10. SPONSORING MONITORING

ATTN: MCHB-DC-EEN

Aberdeen Proving Ground, MD 21010-5422

11. SUPPLEMENTARY NOTES

Copies are available from the National Technical Information Service, 5285 Port Royal Road, Springfield, VA 22161.

12a. DISTRIBUTION / AVAILABILITY STATEMENT

12b. DISTRIBUTION CODE

Approved for public release; distribution is unlimited.

13. ABSTRACT (Maximum 200 words)

\begin{tabular}{|c|c|c|}
\hline $\begin{array}{l}\text { 14. SUBJECT TERMS } \\
\text { acoustics }\end{array}$ & \multicolumn{2}{|c|}{ sound waves -- scattering } \\
\hline $\begin{array}{l}\text { 17. SECURITY CLASSIFICATION } \\
\text { OF REPORT } \\
\text { Unclassified }\end{array}$ & $\begin{array}{l}\text { 18. SECURITY CLASSIFICATION } \\
\text { OF THIS PAGE } \\
\text { Unclassified }\end{array}$ & $\begin{array}{l}\text { 19. SECURITY CLASSIFICATION } \\
\text { OF ABSTRACT } \\
\text { Unclassified }\end{array}$ \\
\hline
\end{tabular}




\section{Foreword}

This study was conducted for USACHPPM under Project 4A161102BT25, "Environmental Research-Corps of Engineers;" Work Unit IE6, "Terrain, Weather and Source Direct Modeling for Blast Noise Propagation." The technical monitor was William Russell, USACHPPM.

The project was carried out under the U.S. Army Research Office Broad Agency Announcement DAAH04-94-R-BAA3 dated January 1994, as part of the U.S. Army Environmental Quality Basic Research Program. The point of contact was Larry L. Pater, Planning and Mission Impact Division (LL-P) of the Land Management Laboratory (LL), U.S. Army Construction Engineering Research Laboratories (USA CERL). The author would like to take this opportunity to thank her advisor, Professor George W. Swenson, Jr., for providing her with the opportunity to work on this project. His encouragement and support have been invaluable to her Master's study at the University of Illinois. She would also like to thank Ms. Alona Boag, who lay the foundation for this project, and Dr. Yuan-Liang Li, who taught her avout the computational methods in acoustics. Dr. Harold E. Balbach is Chief, CECER-LL-P and Dr. William D. Severinghaus is Operations Chief, CECER-LL.

Dr. Michael J. O'Connor is Director of USACERL. 


\section{TABLE OF CONTENTS}

CHAPTER

PAGE

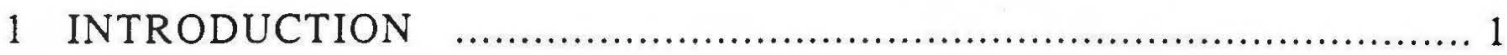

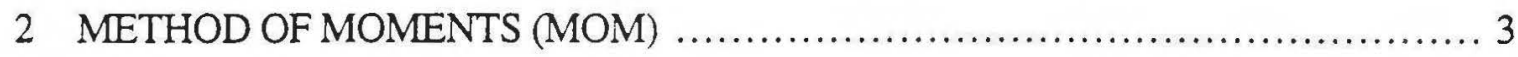

2.1 Mathematical Derivation Of The IE .................................. 3

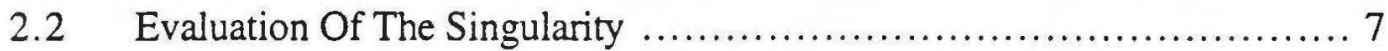

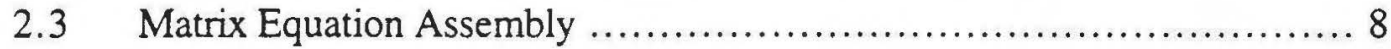

$2.4 \quad$ Impedance Matrix Formulation $\ldots \ldots \ldots \ldots \ldots \ldots \ldots \ldots \ldots \ldots \ldots \ldots \ldots \ldots \ldots$

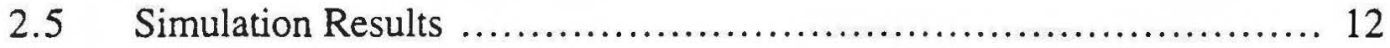

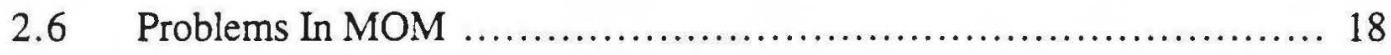

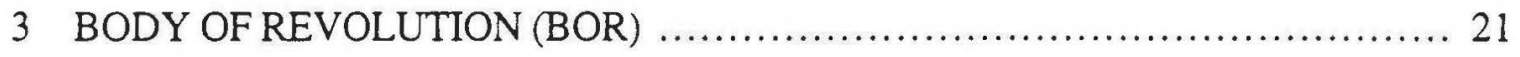

3.1 Derivation Of BOR ........................................... 21

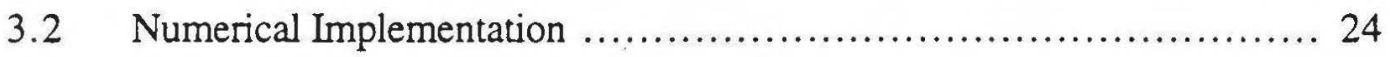

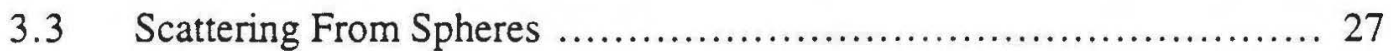

3.4 Scattering From Parabolic Reflectors ............................ 31

3.5 Scattering From Thin Disks ....................................... 36

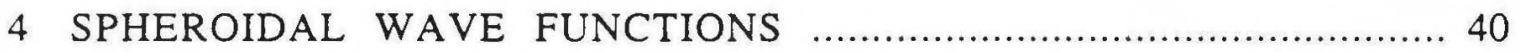

4.1 The Oblate Spheroidal Geometry ................................. 40

4.2 The Angle Functions .............................................. 43

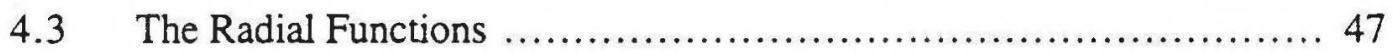

4.4 The Analytic Solutions ......................................... 51

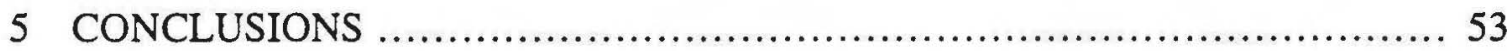

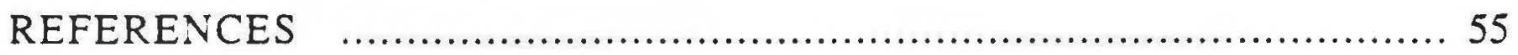

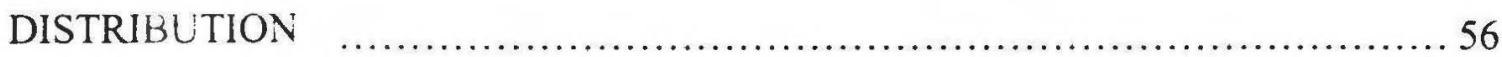




\section{LIST OF FIGURES}

Figure

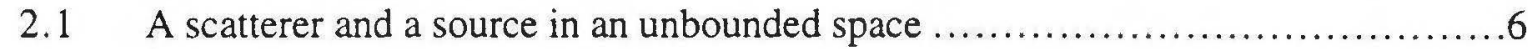

2.2 Limiting procedure....................................................

2.3 The meshed rectangular barrier and the coordinate system $\ldots \ldots \ldots \ldots \ldots \ldots \ldots \ldots$

$2.4 \quad$ Point $\mathrm{P}$ within a triangular element ........................................ 12

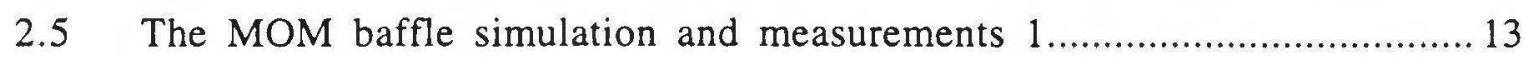

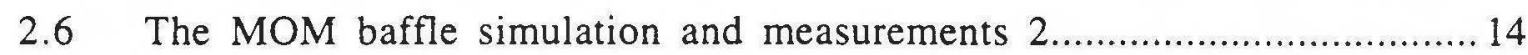

2.7 The tilted baffle........................................................ 14

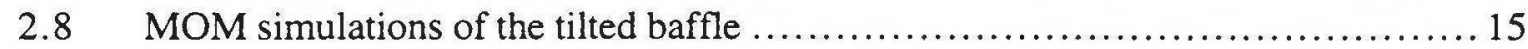

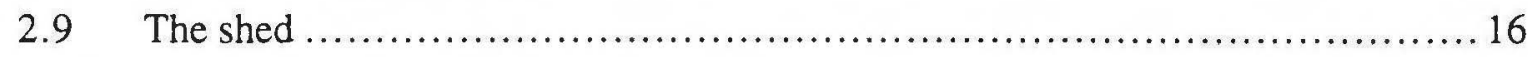

2.10 The MOM simulation of the shed ........................................ 17

2.11 The hill ................................................................ 17

2.12 The MOM simulations of the hill.............................................. 18

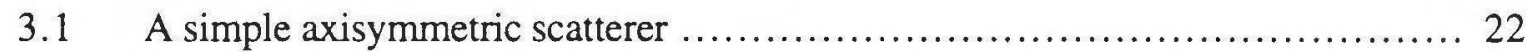

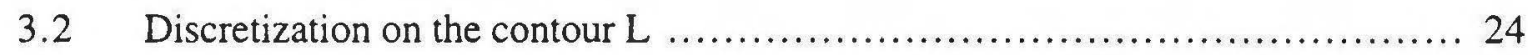

3.3 The element mapping onto the local coordinate system ........................... 25

3.4 Scattering from one sphere ............................................... 28

3.5 The BOR simulation and analytic scattering patterns of the sphere $\ldots \ldots \ldots \ldots \ldots 28$

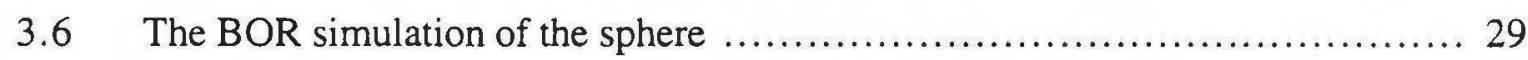

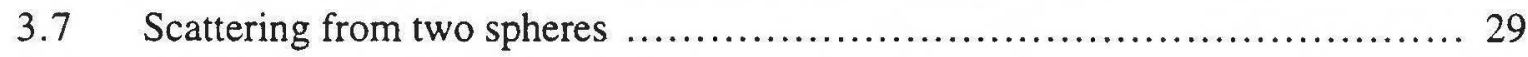

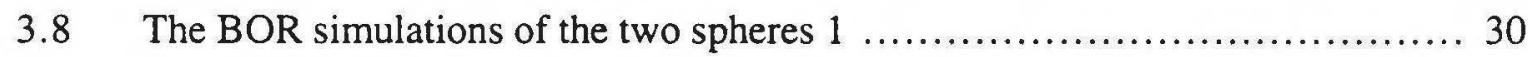

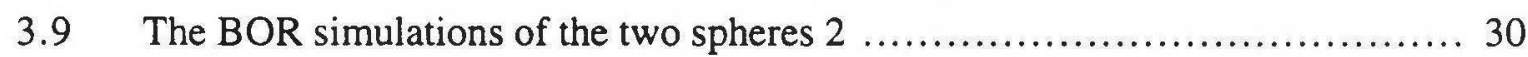

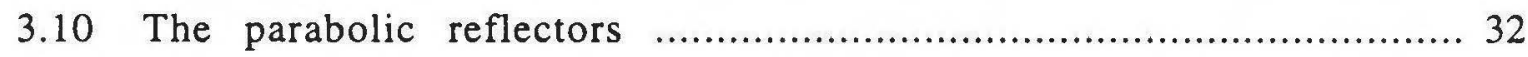

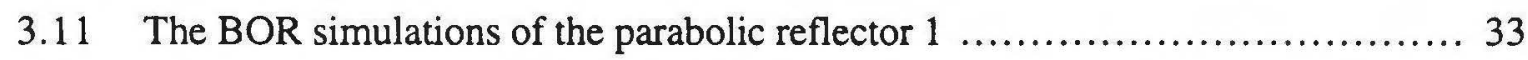

3.12 The BOR simulations of the parabolic reflector 2 .......................... 33

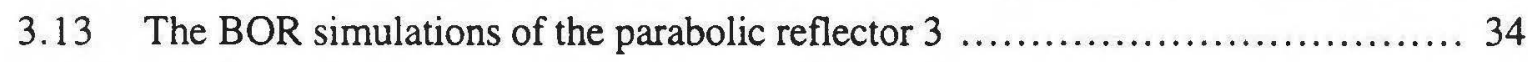

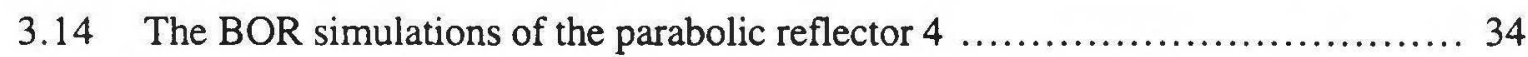

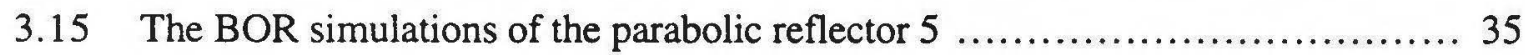

3.16 The BOR simulations of the parabolic reflector, disk, and baffle .............. 35

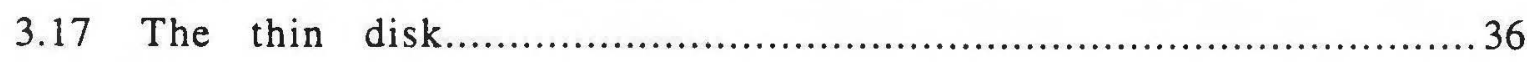




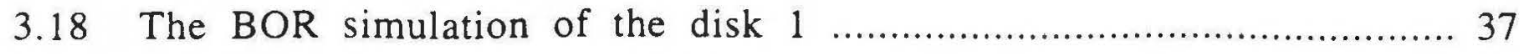

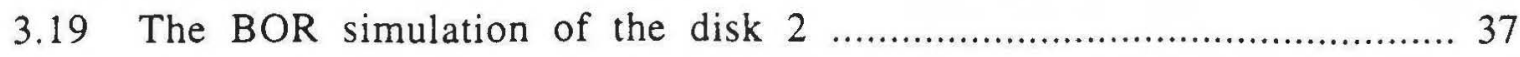

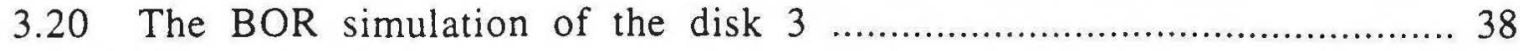

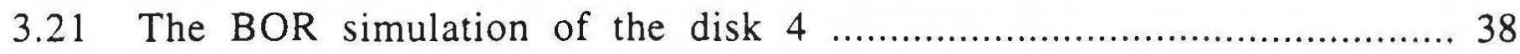

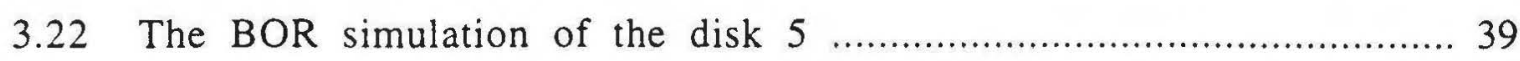

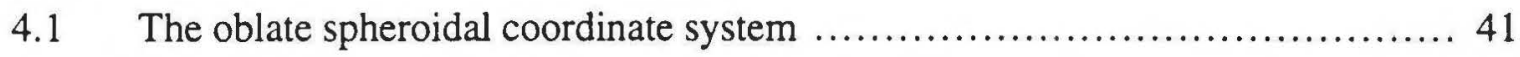

4.2 The BOR simulation and analytic diffraction solution of the disk 1 .............. 52

4.3 The BOR simulation and analytic diffraction solution of the disk 2 ............. 52 



\section{CHAPTER 1 \\ INTRODUCTION}

Loud noises such as gun blast noises from Army training facilities have brought concerns from nearby communities. In response to this environmental issue, the Army has built and implemented noise monitoring systems and noise barriers. For an accurate and effective solution to noise problems, fundamental acoustic scattering and propagation should be carefully studied.

Studying the acoustic scattering has been difficult and incomplete by theoretical means. An efficient and accurate numerical method is necessary to investigate acoustic scattering. A comprehensive investigation was done by Alona Boag under the direction of George W. Swenson, Jr. at United States Army Construction Engineering Research Laboratory (USACERL) Acoustics Team. Alona Boag concluded that method of moments (MOM) was the best approach in solving acoustic scattering problems. MOM involves in solving an integrodifferential equation in a matrix equation form. She has written an MOM code which calculates the radiation patterns of an acoustic source scattered by an object. Her simulation result closely matches experimental data taken from a scattering of a rectangular baffle.

Her MOM program is an excellent way of solving scattering problems of small and simple objects. However her geometry meshing scheme, explained in Chapter 2, becomes tedious when creating curved or complex geometry. To effectively create 3 -D objects, a package called PATRAN has been explored. A few simple PATRAN commands create geometry, mesh surfaces, and output node coordinates.

Another difficulty is in analyzing an object large compared to its wave length, $\lambda$. The number of unknowns to be solved grows as an order of $(d / \lambda)^{2}$, and the central processing unit (CPU) time grows as an order of $(d / \lambda)^{3}$, where $d$ represents a dimension of the object. For axisymmetric geometry, a method called, body of revolution (BOR) technique, 
eliminates such problems. BOR reduces the number of unknowns to an order of $(d / \lambda)$, thereby reducing the CPU time. A BOR code written in FORTRAN is used to analyze scattering from curved, axisymmetric bodies. The BOR code has been extensively tested for its validity, including a comparison to an exact solution for scattering from oblate spheroidal objects involving expansion of the field in series of spheroidal functions.

Chapters 2 and 3 of this thesis present the study of MOM and BOR in detail: mathematical derivations, code implementation, and simulation results. In addition, an in depth study of oblate spheroidal functions is given in chapter 4 . Chapter 5 presents the overall results and conclusion. 


\section{CHAPTER 2 METHOD OF MOMENTS}

There are several methods for solving an acoustic scattering problem. A well known method is geometrical optics (GO). GO yields an approximate solution for scattering from bodies large compared to the acoustic wavelength [1]. Because it is an approximate solution, only applicable to the analysis of high frequency waves, it is inappropriate for USACERL's scattering analysis. For example, a typical spectrum of an Army gun blast noise ranges from $5 \mathrm{~Hz}$ to $60 \mathrm{~Hz}$ [2]. In addition, accurate prediction of scattering from a complicated geometry is almost impossible. The above reasons eliminate the further study of GO.

Another well known scattering mode is Rayleigh scattering. Rayleigh scattering is typically used to analyze scattering from small particles such as air bubbles in liquids and water molecules in the air. The dimensions of practical noise barriers are comparable to or larger than the acoustic wavelength, so Rayleigh scattering is not an appropriate model.

Alona Boag has investigated two rigorous numerical methods in solving acoustic scattering: a partial differential equation method (PDE) and an integral equation method (IE). She presented her findings at a USACERL seminar, and Table 2.1 summarizes her findings. Table 2.1 clearly indicates that the IE method is the best choice among available scattering solutions. The IE method leads to MOM, and the remainder of this chapter is devoted to MOM.

\subsection{Mathematical Derivation of The IE}

When an acoustic source radiates a wave in free space, the radiated field $\psi$ at an observation point $\mathbf{r}$ can be mathematically described as :

$$
\psi(\mathbf{r})=-\int_{V} f\left(\mathbf{r}_{\mathbf{s}}\right) \mathrm{G}\left(\mathbf{r}, \mathbf{r}_{\mathbf{s}}\right) \mathrm{dv}
$$

where $f$ is the source at $\mathbf{r}_{\mathbf{s}}$. The Green's function $\mathrm{G}\left(\mathbf{r}, \mathbf{r}_{\mathbf{s}}\right)$ is an impulse response at $\mathbf{r}$ due to 
$\mathrm{f}\left(\mathbf{r}_{\mathbf{s}}\right)$. In a homogeneous medium,

$$
\mathrm{G}\left(\mathbf{r}, \mathbf{r}_{\mathbf{s}}\right)=\frac{\exp (\mathrm{ikR})}{4 \pi R}
$$

where $\mathrm{R}=\left|\mathbf{r}-\mathbf{r}_{\mathrm{s}}\right|$. The wave number $\mathrm{k}$ is equal to $2 \pi / \lambda$. The integration is taken over the entire volume $v$ of the source. Unless otherwise noted, time dependence $\exp (-i \omega t)$ is assumed.

Table 2.1 Alona Boag's comparison between PDE and IE

\begin{tabular}{|c|c|c|}
\hline Method & Partial Differential Equation & Integral Equation \\
\hline Advantages & $\begin{array}{l}\text { applicable to bodies comparable to } \\
\text { or smaller than the wavelength } \\
\text { - can analyze arbitrary media and } \\
\text { geometry, including } \\
\text { inhomogeneous bodies } \\
\text { - sparse matrices to invert }\end{array}$ & $\begin{array}{l}\text { - IE is based on the Sommerfeld } \\
\text { Radiation Condition } \\
\text { - the radiation condition } \\
\text { automatically satisfied } \\
\text { - lower number of unknowns } \\
\text { compared to PDE method } \\
\text { - high accuracy }\end{array}$ \\
\hline Disadvantages & $\begin{array}{l}\text { number of unknown } N \text { grows as } \\
(\mathrm{d} / \lambda)^{3} \\
\text { - requires artificial boundary } \\
\text { conditions to truncate the mesh } \\
\text { - medium accuracy }\end{array}$ & $\begin{array}{l}\text { - number of unknown } N \text { grows as } \\
(\mathrm{d} / \lambda)^{2} \\
\text { - applicable only to piecewise } \\
\text { homogeneous problems } \\
\text { - CPU time grows as }(\mathrm{d} / \lambda)^{3}\end{array}$ \\
\hline
\end{tabular}

In the presence of an object in the medium, a portion of the radiated wave encounters the object and re-radiates into different directions. The object acts as a scatterer of the original wave. When a field is measured at $\mathbf{r}$, it is a sum of the fields due to the original wave and the re-radiated one. The field due to the original wave is called an incident field, and the other field due to the re-radiated one is called a scattered field. The sum of the incident field and the scattered is the total field. To distinguish each field, the incident, scattered, and total fields at $\mathbf{r}$ are denoted as $\mathrm{P}^{\mathrm{inc}}, \mathrm{P}^{\mathrm{s}}$, and $\mathrm{P}$ respectively. Thus the field in (2.1) should be re-expressed as the incident field Pinc. The incident field is recalculated using the Green's function assuming that the presence of the scatterer does not influence the propagation of the original wave. 
The homogeneous Green's function satisfies the Helmholtz equation, i.e., the frequency domain wave equation:

$$
\left(\nabla^{2}+\frac{\omega^{2}}{c^{2}}\right) \psi(\mathbf{r})=f\left(\mathbf{r}_{\mathbf{s}}\right)
$$

The speed of the sound is denoted as c and is assumed to be $340 \mathrm{~m} / \mathrm{sec}$ in the air. Substituting (2.1) in (2.3) yields,

$$
\left(\nabla^{2}+\frac{\omega^{2}}{c^{2}}\right) G\left(\mathbf{r}, \mathbf{r}_{\mathbf{s}}\right)=-\delta\left(\mathbf{r}-\mathbf{r}_{\mathbf{s}}\right)
$$

Multiplying (2.3) by $\mathrm{G}\left(\mathbf{r}, \mathbf{r}_{\mathbf{s}}\right)$ and (2.4) by $\psi\left(\mathbf{r}, \mathbf{r}_{\mathbf{s}}\right)$ and rearranging terms in each equation, the following relations are obtained:

$$
\begin{gathered}
G\left(\mathbf{r}, \mathbf{r}_{\mathbf{s}}\right) \nabla^{2} \psi(\mathbf{r})=\mathrm{G}\left(\mathbf{r}, \mathbf{r}_{\mathbf{s}}\right)\left(\mathrm{f}\left(\mathbf{r}_{\mathbf{s}}\right)-\frac{\omega^{2}}{\mathrm{c}^{2}} \psi(\mathbf{r})\right) \\
\psi(\mathbf{r}) \nabla^{2} \mathrm{G}\left(\mathbf{r}, \mathbf{r}_{\mathbf{s}}\right)=-\psi(\mathbf{r})\left(\delta\left(\mathbf{r}-\mathbf{r}_{\mathbf{s}}\right)+\frac{\omega^{2}}{c^{2}} \mathrm{G}\left(\mathbf{r}, \mathbf{r}_{\mathbf{s}}\right)\right)
\end{gathered}
$$

The Green's second identity states that for arbitrary scalars U and V,

$$
\int_{\Omega}\left(U \nabla^{2} V-V \nabla^{2} U\right) d \Omega=\int_{S}\left(U \frac{\partial V}{\partial n}-V \frac{\partial U}{\partial n}\right) d S
$$

where $\Omega$ is a volume and $\mathrm{S}$ is the surface enclosing $\Omega$. Replacing $\mathrm{U}$ with the pressure field, $\mathrm{V}$ with the Green's function and using relations (2.5) and (2.6),

$$
\begin{gathered}
-\int_{\Omega}\left[\psi(\mathbf{r})\left(\delta\left(\mathbf{r}-\mathbf{r}_{\mathbf{s}}\right) \mathrm{G}\left(\mathbf{r}, \mathbf{r}_{\mathbf{s}}\right)+\frac{\omega^{2}}{\mathrm{c}^{2}} \mathrm{G}\left(\mathbf{r}, \mathbf{r}_{\mathbf{s}}\right)\right)+\mathrm{G}\left(\mathbf{r}, \mathbf{r}_{\mathbf{s}}\right)\left(\mathrm{f}\left(\mathbf{r}_{\mathbf{s}}\right)-\frac{\omega^{2}}{\mathrm{c}^{2}} \psi(\mathbf{r})\right)\right] \mathrm{d} \Omega . \\
=-\int_{S}\left(\psi\left(\mathbf{r}^{\prime}\right) \frac{\partial \mathrm{G}\left(\mathbf{r}, \mathbf{r}^{\prime}\right)}{\partial \mathrm{n}}-\mathrm{G}\left(\mathbf{r}, \mathbf{r}^{\prime}\right) \frac{\partial \psi\left(\mathbf{r}^{\prime}\right)}{\partial \mathrm{n}}\right) \mathrm{d} S
\end{gathered}
$$

where $\psi\left(\mathbf{r}^{\prime}\right)$ is the field on the surface and the partial derivatives were taken with respect to a normal vector on the surface. The normal vector $\mathbf{n}$ points outward from the volume $\Omega$. Note that the minus sign at the right hand side of (2.7) reflects that $-\mathbf{n}$ is taken instead of $\mathbf{n}$ (see 
Figure 2.1). The surface includes the scatterer surface and the fictitious surface at infinity (see Figure 2.1).

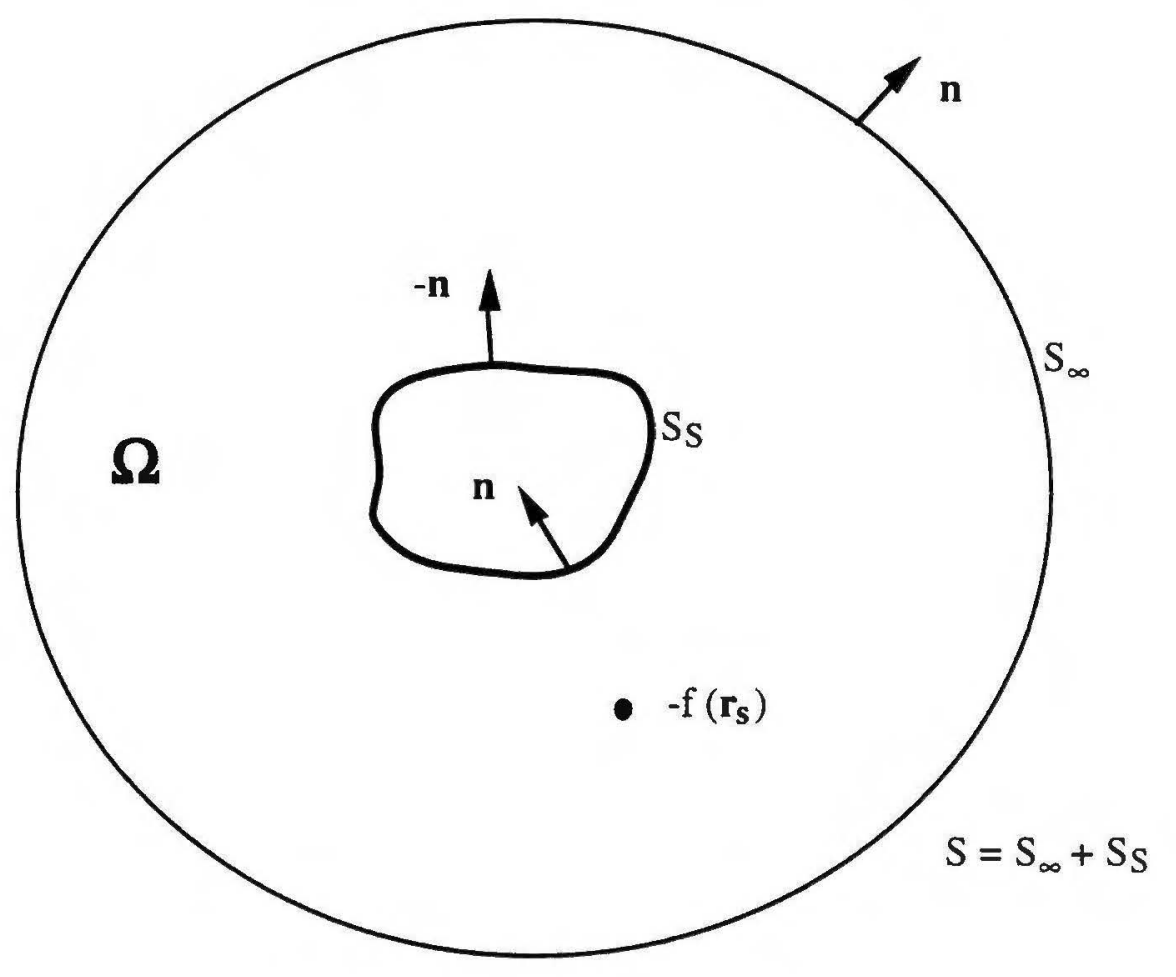

Figure 2.1 A scatterer and a source in an unbounded space

After substituting (2.5) and (2.6) on the left hand side of (2.8) and simplifying, (2.8) becomes,

$$
-\psi(\mathbf{r})-\int_{\Omega} G\left(\mathbf{r}, \mathbf{r}_{\mathbf{s}}\right) f\left(\mathbf{r}_{\mathbf{s}}\right) \mathrm{d} \Omega=-\int_{S}\left(-\psi\left(\mathbf{r}^{\prime}\right) \frac{\partial G\left(\mathbf{r}, \mathbf{r}^{\prime}\right)}{\partial n}-G\left(\mathbf{r}, \mathbf{r}^{\prime}\right) \frac{\partial \psi\left(\mathbf{r}^{\prime}\right)}{\partial n}\right) d S
$$

By equation (2.1), the integral on the left hand side of (2.9) is essentially equal to the incident field $\mathrm{P}^{\mathrm{inc}}$.

All fields in any unbounded medium must satisfy the Sommerfeld radiation condition:

$$
\left(\frac{\partial}{\partial \mathrm{R}}-\mathrm{ik}\right) \psi=0 \text { as } \mathrm{R} \rightarrow \infty
$$

This condition ensures that the field $\psi$ becomes negligible at a large distance from the 
acoustic source. Applying the radiation condition to (2.10) eliminates the field contribution from the fictitious surface. Thus the volume integral is reduced to a surface integral:

$$
P(\mathbf{r})=P^{i n c}(\mathbf{r})+\int_{S_{S}}\left(P\left(\mathbf{r}^{\prime}\right) \frac{\partial G\left(\mathbf{r}, \mathbf{r}^{\prime}\right)}{\partial n}-G\left(\mathbf{r}, \mathbf{r}^{\prime}\right) \frac{\partial P\left(\mathbf{r}^{\prime}\right)}{\partial n}\right) d S_{S}
$$

where $\psi(\mathbf{r})$ and $\psi\left(\mathbf{r}^{\prime}\right)$ are re-expressed as the pressure field $\mathrm{P}(\mathbf{r})$ at $\mathbf{r}$ and $\mathrm{P}\left(\mathbf{r}^{\prime}\right)$ at $\mathbf{r}^{\prime}$ respectively.

$\mathrm{P}(\mathbf{r}), \mathrm{P}\left(\mathbf{r}^{\prime}\right)$, and the partial derivative of $\mathrm{P}\left(\mathbf{r}^{\prime}\right)$ are the three unknowns to be solved. The surface is assumed to be perfectly rigid. Rigid surfaces do not move in the presence of acoustic pressure, thus the normal velocity field on the surfaces is zero. The pressure field on the surface is doubled, and its partial derivative normal to the surface is zero. Finally the IE of interest is

$$
P(\mathbf{r})=P^{\operatorname{inc}}(\mathbf{r})+\int_{S_{S}} P\left(r^{\prime}\right) \frac{\partial G\left(r, r^{\prime}\right)}{\partial n} d S_{S}
$$

(2.12) will be used to build a matrix equation that can be solved in a computer program.

\subsection{Evaluation of The Singularity}

The equation (2.12) can be evaluated at any observation point $\mathbf{r}$, and when $\mathbf{r}$ approaches the surface of the scatterer, the integral in (2.12) becomes singular at $\mathbf{r}=\mathbf{r}$ '. Morita, et. al. cleverly evaluate the integral at the singularity [3].

Take an infinitesimally small surface around $\mathbf{r}^{\prime}$ and call it $\delta S$. For a convenient evaluation of the singularity, shift the coordinate system such that the observation point $\mathbf{r}$ and the integration point $\mathbf{r}^{\prime}$ are $(0,0, z)$ and $\left(\rho^{\prime}, \phi^{\prime}, z^{\prime}\right)$, respectively (see Figure 2.2$)$ and set $z^{\prime}=0$. The integral is then approximated as,

$$
\int_{\delta S} \mathrm{P}\left(\mathbf{r}^{\prime}\right) \frac{\partial \mathrm{G}\left(\mathbf{r}, \mathbf{r}^{\prime}\right)}{\partial \mathrm{n}} \mathrm{dS}=\int_{\delta S} \mathrm{P}\left(\mathbf{r}^{\prime}\right) \frac{\partial\left(\frac{\exp \left(\mathrm{ik}\left|\mathbf{r}-\mathbf{r}^{\prime}\right|\right.}{4 \pi\left|\mathbf{r}-\mathbf{r}^{\prime}\right|}\right)}{\partial \mathrm{n}} \mathrm{dS} \approx \int_{\delta S} \mathrm{P}\left(\mathbf{r}^{\prime}\right) \frac{\partial\left(\frac{1}{4 \pi\left|\mathbf{r}-\mathbf{r}^{\prime}\right|}\right)}{\partial \mathrm{n}} \mathrm{dS}
$$


At $\mathbf{r} \approx \mathbf{r}^{\prime}, \mathrm{P}\left(\mathbf{r}^{\prime}\right)$ is approximately equal to $\mathrm{P}(\mathbf{r})$ and may be assumed invariant within $\delta \mathrm{S}$. Figure 2.2 indicates that $-\mathbf{z}$ is the normal vector, and taking a derivative with respect to $\mathbf{z}$ yields,

$$
\frac{1}{4 \pi} P(\mathbf{r}) \int_{0}^{a} \frac{\partial}{\partial z^{\prime}}\left(\frac{1}{\sqrt{\rho^{\prime 2}+\left(z-z^{\prime}\right)^{2}}}\right) 2 \pi \rho^{\prime} d \rho^{\prime}=-\frac{1}{2} P(\mathbf{r})\left(\frac{z}{\sqrt{a^{2}+z^{2}}}-\frac{z}{|z|}\right)
$$

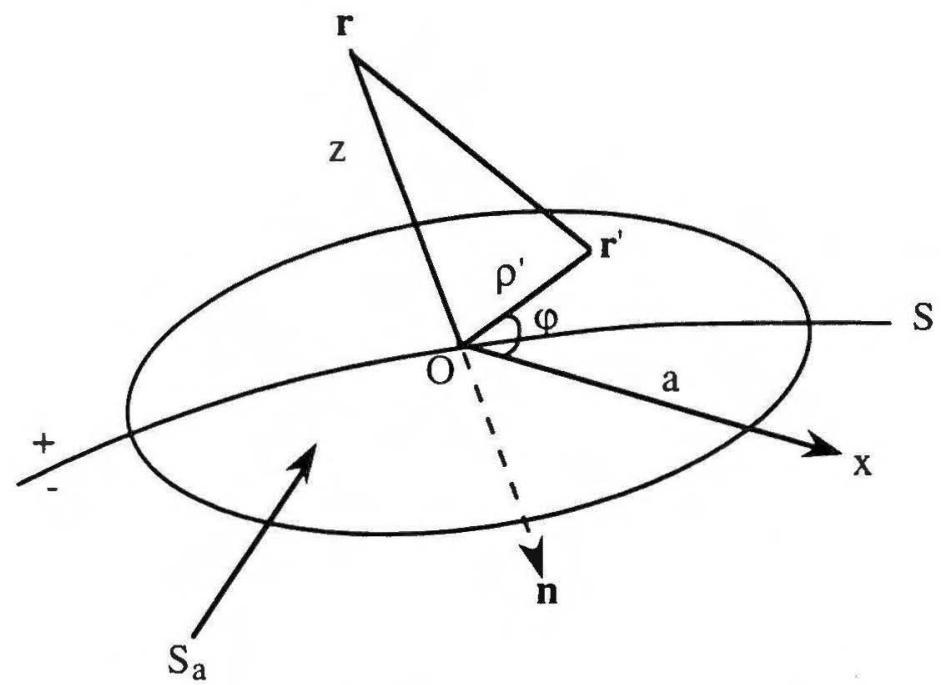

Figure 2.2 Limiting procedure

As a and $z$ approach zero, the integral at the singularity is reduced to $-\frac{1}{2} P(\mathbf{r})$. Therefore when the observation point is taken on the surface of the scatterer, (2.12) should be rewritten as:

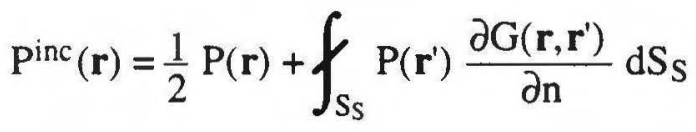

where $f$ indicates the principal value of the integral. The above equation is solved to find the pressure fields on the surface by assembling (2.15) into a matrix equation. Once the pressure fields on the surface are known, then the real IE (2.12) can be solved for any observation location.

\subsection{Matrix Equation Assembly}

Assembling a matrix equation from IE is the key step in MOM. MOM is a technique 
which solves the IE by dividing the scatterer surface into small patches called elements. The elements can be any shape as long as the shape can be used tightly to mesh the surface, leaving no "holes" or space on it. The simulation program written by Alona Boag uses triangular elements to mesh the surface. For accurate simulation results, the lengths of three sides of each triangular element must be approximately equal, i.e., isometric. Creating an element with one extremely long leg compared to the others is not recommended. Also the length of each triangle leg, denoted by grid length, should be less than one tenth of a wave length.

Assuming the pressure fields do not very drastically within each element, $P\left(\mathbf{r}^{\prime}\right)$ in (2.15) can be regarded constant on each element. Usually the value of $\mathrm{P}\left(\mathbf{r}^{\prime}\right)$ on the center of each element is picked; this is how the method of moment is named. The process of assembling a matrix equation is described for a typical rectangular barrier (see Figure 2.3).
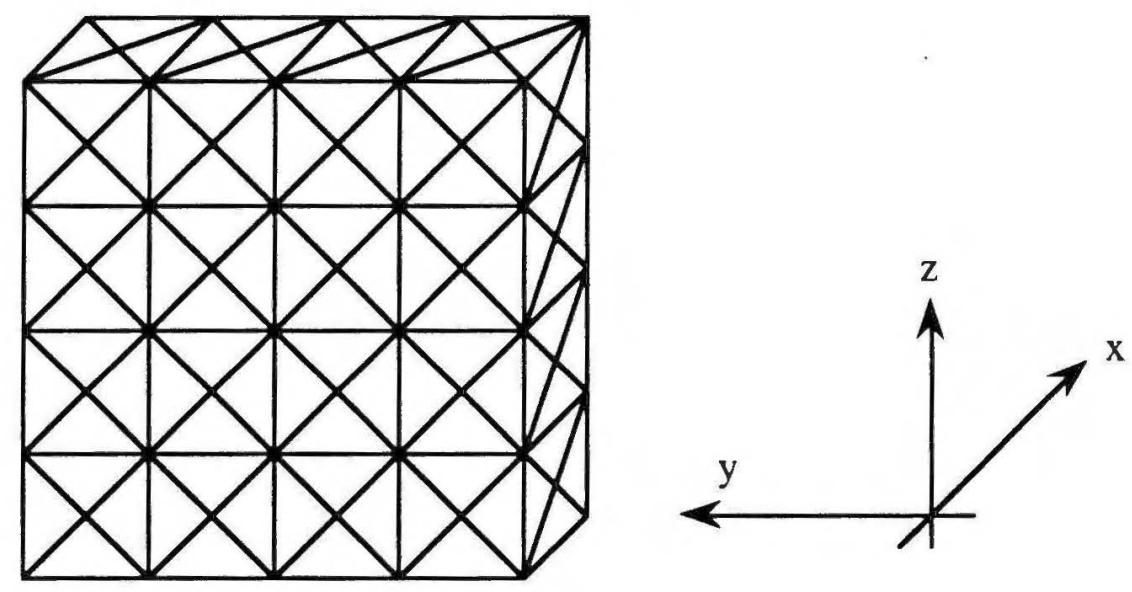

Figure 2.3 The meshed rectangular barrier and the coordinate system

Let $\mathrm{N}$ be the total number of the triangular elements on the surface, and define pulse basis functions

$$
\begin{array}{lll}
f_{j}=1, & \text { on } \Delta S_{j}^{\prime} & , i \neq j \\
f_{j}=0, & \text { on all other } \Delta S_{i}^{\prime} &
\end{array}
$$

where $\Delta S^{\prime}{ }_{i}$ is the $j^{\text {th }}$ triangular element on the surface. Let $\mathbf{r}_{j}{ }_{j}$ be the center coordinate of 
$\Delta S^{\prime}{ }_{i}$. Also let the unknown pressure fields on the surface be expressed by,

$$
P\left(\mathbf{r}^{\prime}\right)=\sum_{i=1}^{N} p_{j} f_{j}
$$

where $\mathbf{p}$ is a $1 \times \mathbf{N}$ unknown array for the surface pressure fields. Substituting (2.17) into (2.15), and matching the field at the midpoint of each $\Delta$ 's, a matrix equation is obtained as below:

$$
p_{i}^{i n c}=\sum_{i=1}^{N} Z_{i j} p_{j} \quad, i=1,2,3, \cdots, N
$$

Expressing (2.18) in a matrix form,

$$
[\mathbf{p}]=\left[\begin{array}{ll}
\mathbf{Z} &
\end{array}\right]\left[\mathbf{p}^{\text {inc }}\right]
$$

where

$$
\begin{array}{ll}
Z_{i j}=\int_{\Delta S_{j}} \frac{\partial G\left(\mathbf{r}_{i}, \mathbf{r}^{\prime}\right)}{\partial n} d S & , i \neq j \\
Z_{i j}=\frac{1}{2} & , i=j
\end{array}
$$

The derivative of the Green's function with respect to a normal vector is

$$
\frac{\partial G}{\partial n}=\left(i k-\frac{1}{\left|\mathbf{r}_{\mathbf{i}}-\mathbf{r}^{\prime}\right|}\right) \frac{\exp \left(\mathrm{ik} \mid \mathbf{r}_{\mathbf{i}}-\mathbf{r}^{\prime}\right)}{4 \pi\left|\mathbf{r}_{\mathbf{i}}-\mathbf{r}^{\prime}\right|}\left(\mathbf{r}_{\mathbf{i}}-\mathbf{r}^{\prime}\right) \cdot \mathbf{n}
$$

The $\mathbf{N} \times \mathbf{N}$ matrix $\mathbf{Z}$ is called an impedance matrix, and it needs be inverted to find the unknown surface pressures in $\mathbf{p}$.

$$
\mathrm{p}_{j}=\sum_{\mathrm{i}=1}^{\mathrm{N}} Z_{\mathrm{ij}}^{1} \mathrm{p}_{\mathrm{i}}^{\mathrm{inc}}
$$

To find a total pressure at any location $\mathbf{r}$, sum the incident field and the scattered field found by the above method; 


$$
P(\mathbf{r})=P^{i n c}(\mathbf{r})+\sum_{j=1}^{N} p_{j} \int_{\Delta S_{j}} \frac{\partial G\left(\mathbf{r}, \mathbf{r}_{\mathbf{j}}\right)}{\partial n} d S
$$

\subsection{Impedance Matrix Formulation}

Finding values of the $\mathbf{Z}$ matrix is the central part for solving the matrix equation. Each matrix element $Z_{i j}$ represents the contribution of the surface integration of $j^{\text {th }}$ element at the center of $i^{\text {th }}$ element. The surface integration is numerically done, and its accuracy is very critical to the overall accuracy of MOM. The seven-point Gaussian quadrature is used for the numerical integration; an integration of any 'smooth' function within a triangle can be accurately obtained by sampling seven points within the triangle.

$$
\int_{\text {triangle }} f(\mathbf{r}) d s=A \sum_{i=1}^{7} w_{i} f\left(r_{i}\right)+\mathbf{O}\left(h^{6}\right)
$$

where $A$ is the area of the equilateral triangle and $w_{i}$ is the weighting value. The error of the quadrature is on the sixth order of $h$, the radius of a circle circumscribing the triangle, so $h$ must be less than 1 for accuracy. A point $\mathrm{P}$ inside a triangle can be uniquely expressed in terms of the areas of subtriangles defined by $\mathrm{P}$ and the vertices of the main triangle (see Figure 2.4). The subtriangles L1, L2, and L3 are called area coordinates and they add up to 1. The area coordinates and weights for the seven points are listed in Table 2.2 [4]. The coordinates for the point inside the triangle can be expressed as

$$
\mathbf{r}_{\mathbf{i}}=\mathbf{P} 1 \mathrm{~L} 1+\mathbf{P} 2 \mathrm{~L} 2+\mathbf{P} 3 \mathrm{~L} 3
$$

where $\mathbf{P 1}, \mathbf{P} 2, \mathbf{P} 3$ are the three vertices coordinates of the triangle. 


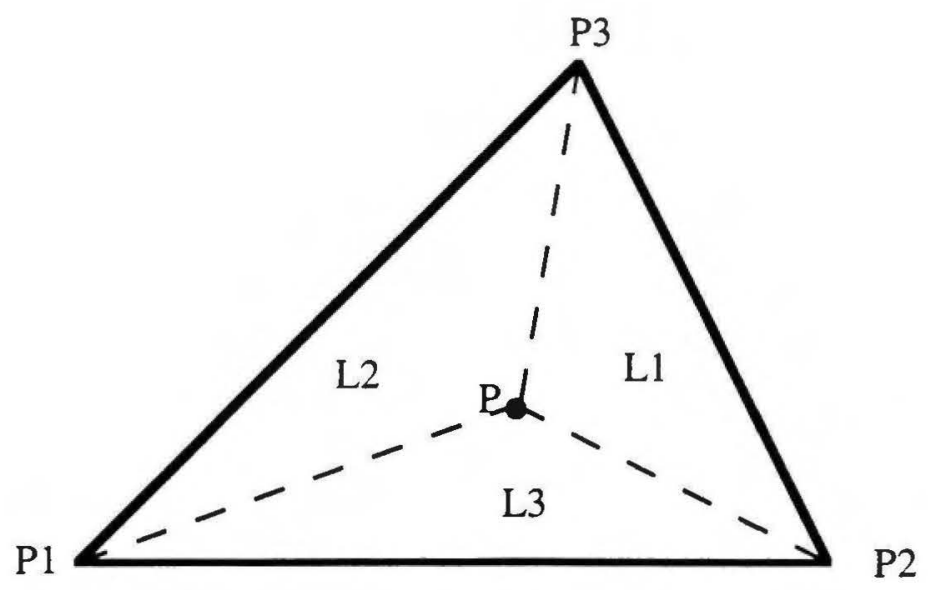

Figure 2.4 Point $\mathrm{P}$ within a triangular element

Table 2.2 The area coordinates and weights

\begin{tabular}{|c|c|c|c|c|}
\hline $\mathrm{i}$ & L1 & $\mathrm{L} 2$ & $\mathrm{~L} 3$ & $\mathrm{w}_{\mathrm{i}}$ \\
\hline 1 & $\frac{1}{3}$ & $\frac{1}{3}$ & $\frac{1}{3}$ & $\frac{270}{1200}$ \\
\hline 2 & $\frac{(9+2 \sqrt{15})}{21}$ & $\frac{(4-\sqrt{15})}{21}$ & $\frac{(7-\sqrt{15})}{21}$ & $\frac{(155-\sqrt{15})}{1200}$ \\
\hline 3 & $\frac{(4-\sqrt{15})}{21}$ & $\frac{(9+2 \sqrt{15})}{21}$ & $\frac{(7-\sqrt{15})}{21}$ & $\frac{(155-\sqrt{15})}{1200}$ \\
\hline 4 & $\frac{(4-\sqrt{15})}{21}$ & $\frac{(4-\sqrt{15})}{21}$ & $\frac{(13+2 \sqrt{15})}{21}$ & $\frac{(155-\sqrt{15})}{1200}$ \\
\hline 5 & $\frac{(9-2 \sqrt{15})}{21}$ & $\frac{(4+\sqrt{15})}{21}$ & $\frac{(7+\sqrt{15})}{21}$ & $\frac{(155+\sqrt{15})}{1200}$ \\
\hline 6 & $\frac{(4+\sqrt{15})}{21}$ & $\frac{(9-2 \sqrt{15})}{21}$ & $\frac{(7+\sqrt{15})}{21}$ & $\frac{(155+\sqrt{15})}{1200}$ \\
\hline 7 & $\frac{(4+\sqrt{15})}{21}$ & $\frac{(4+\sqrt{15})}{21}$ & $\frac{(13-2 \sqrt{15})}{21}$ & $\frac{(155+\sqrt{15})}{1200}$ \\
\hline
\end{tabular}

\subsection{Simulation Results}

To verify the accuracy of the MOM program, a simulation of scattering from a wooden baffle was run and compared to the physical measurements performed outdoors. 
The baffle was $61 \mathrm{~cm}$ wide and $30.5 \mathrm{~cm}$ high, and a microphone was placed at the horizontal center, about $7.6 \mathrm{~cm}$ above the ground, and $2.5 \mathrm{~cm}$ away from the face of the baffle. The experimental data are taken from Benson et al. [5]. In the numerical analysis a point source is placed at the microphone position using the reciprocity theorem. The simulation results closely match the measurements (see Figures 2.5 and 2.6). Also diffraction patterns for the same baffle but mounted at a tilt angle have been simulated (see Figures 2.7 and 2.8).

Total pressure field amplitude in the azimuthal plane

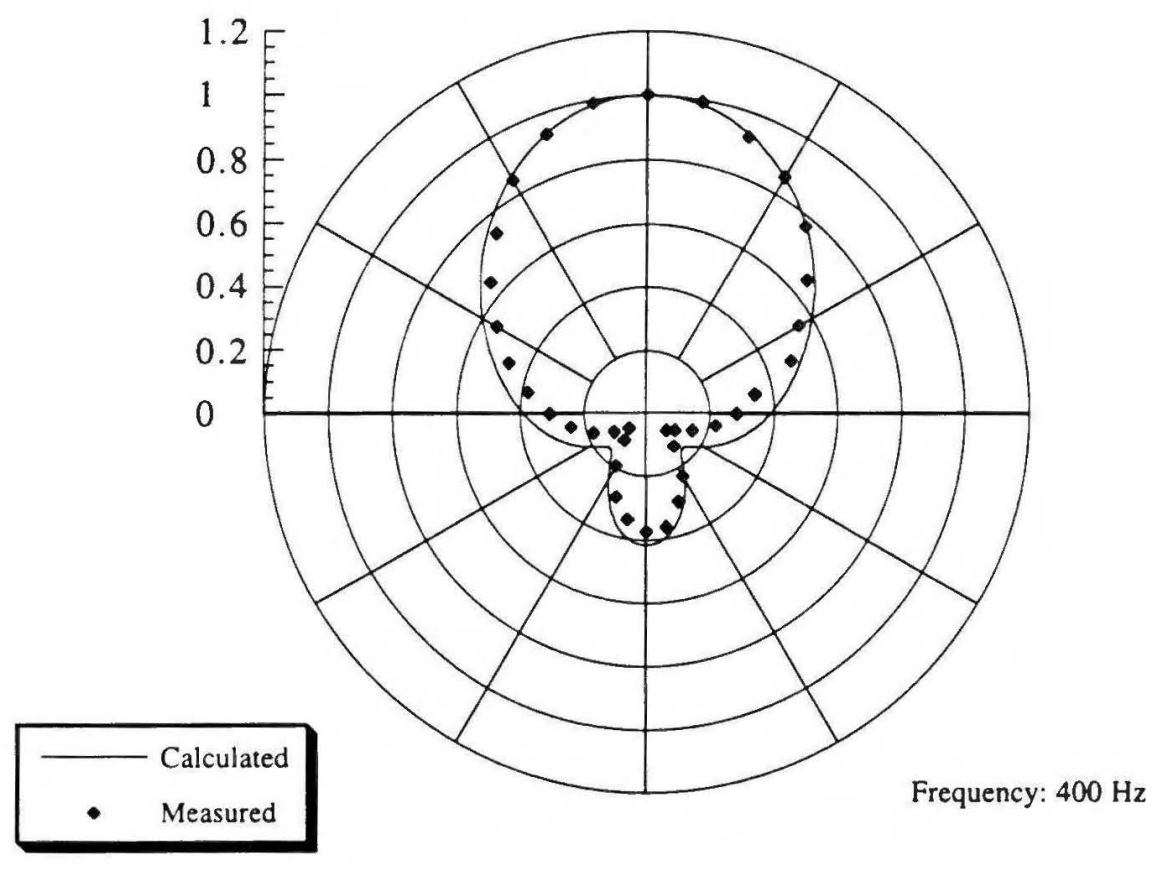

Figure 2.5 The MOM baffle simulation and measurements 1 
Total pressure field amplitude in the azimuthal plane

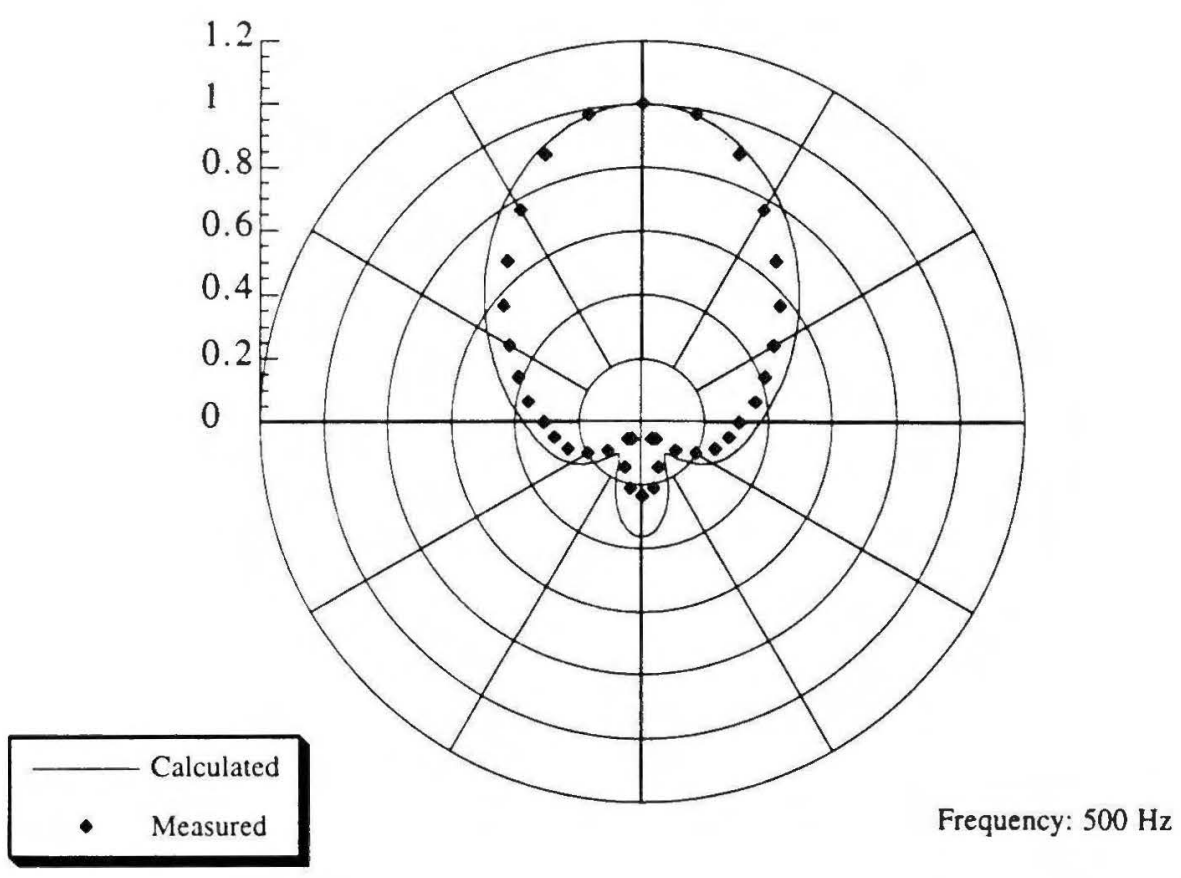

Figure 2.6 The MOM baffle simulation and measurements 2

Side view

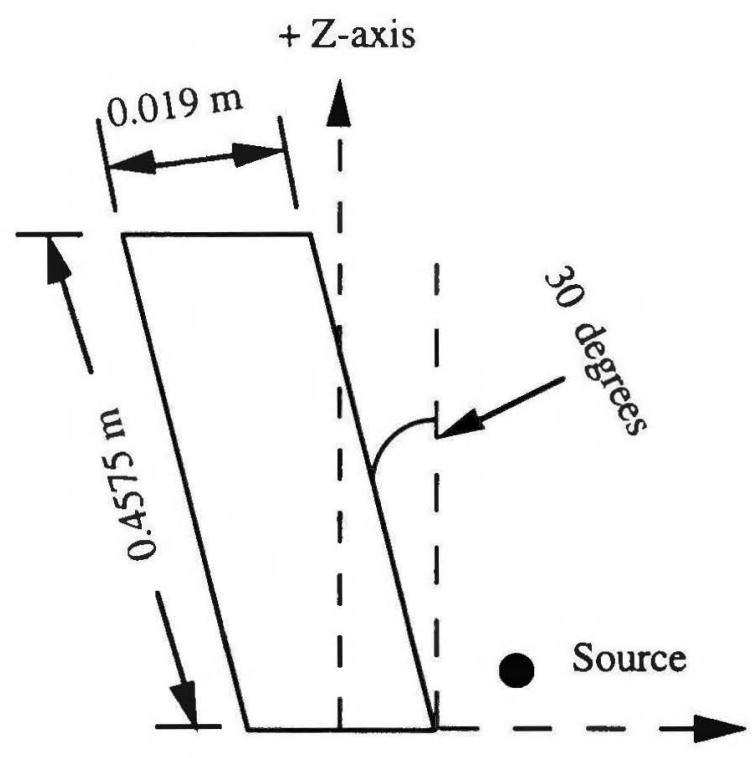

Figure 2.7 The tilted baffle 
Total pressure field amplitude in the azimuthal plane

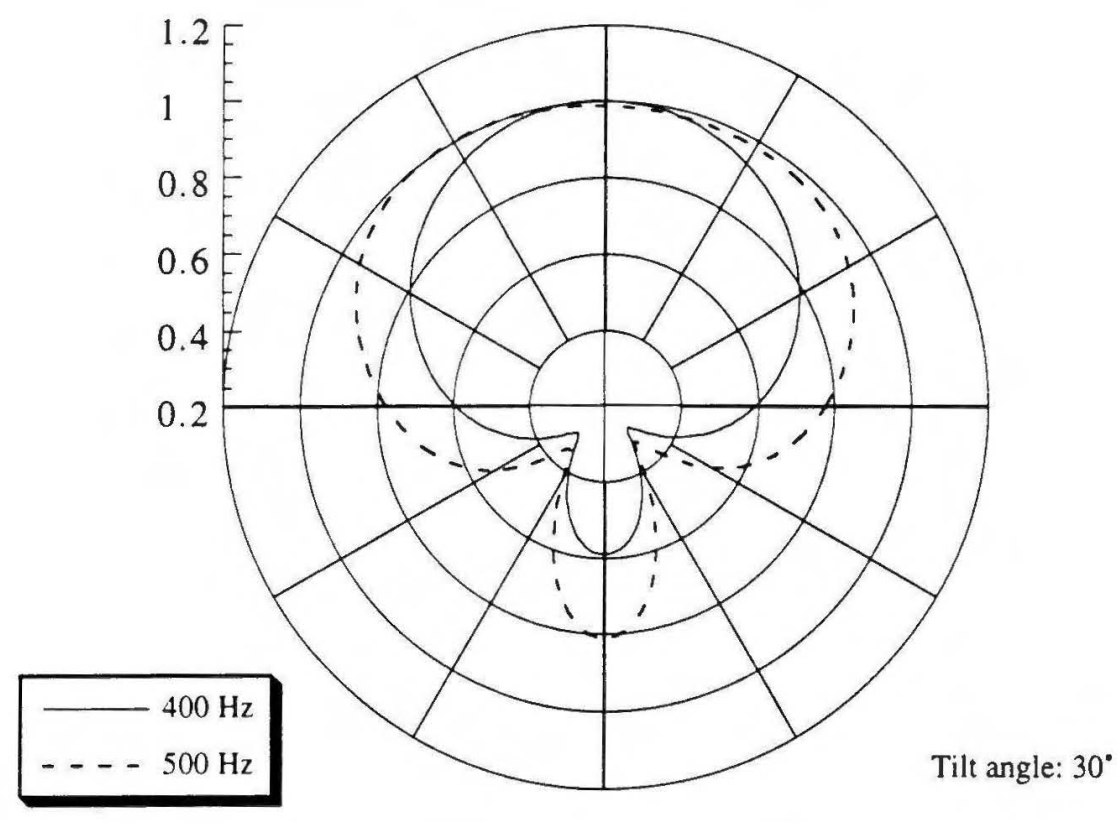

Figure 2.8 MOM simulations of the tilted baffle 2

When one fires a gun inside a shed, the acoustic excitation may be represented as a point source. A simple shed has been modeled as in Figure 2.9, and the diffraction patterns have been studied. Figure 2.10 shows the diffraction pattern of a point source inside a shed. Also a simple hill has been modeled to see the diffraction patterns of gun blast noises nearby a hill (see Figures 2.11 and 2.12). The dimensions of the hill is small compared to a realistic hill, and analyzing a larger hill requires more CPU time and memory. 

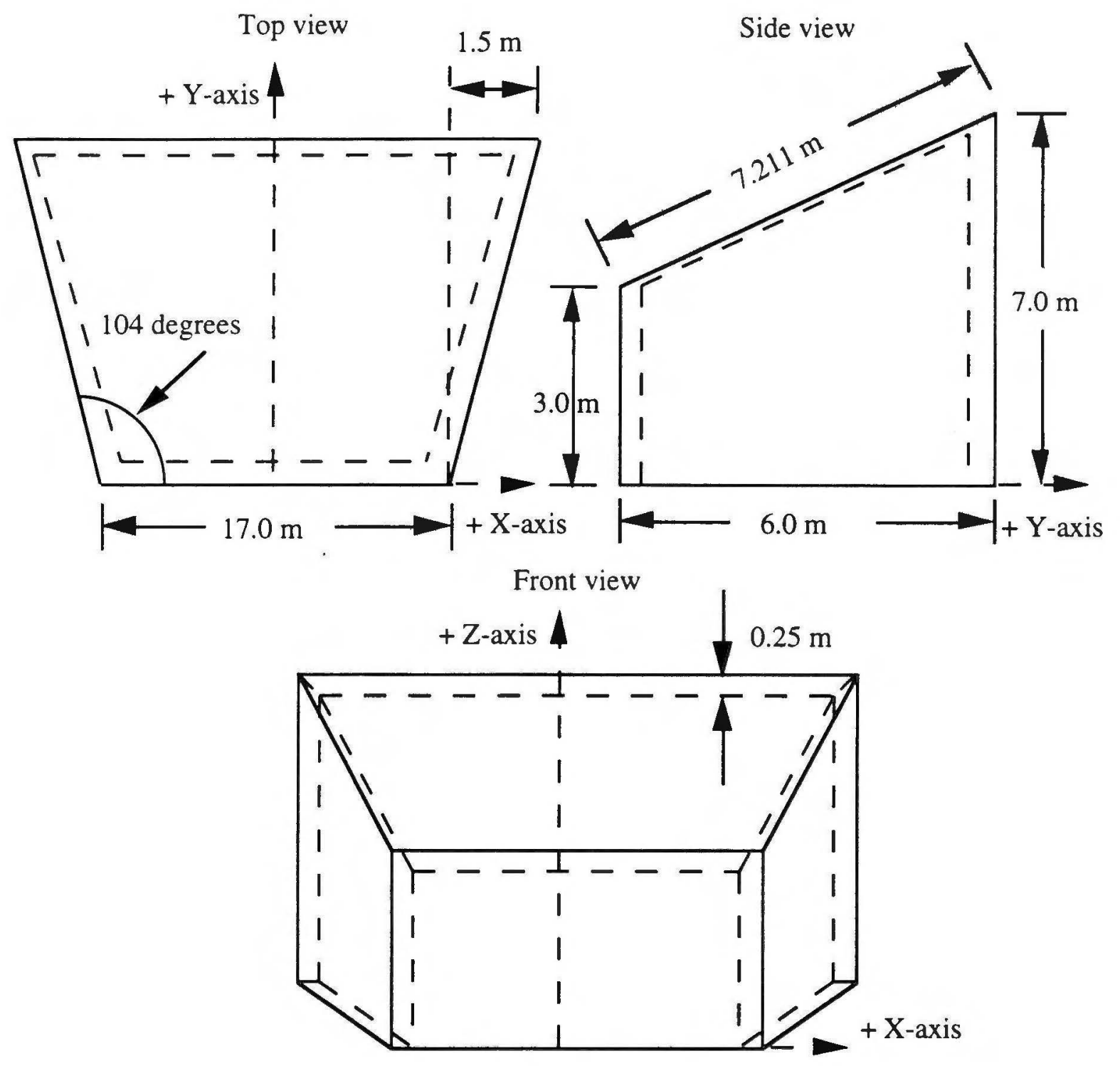

Figure 2.9 The shed 
Total pressure field amplitude in the azimuthal plane

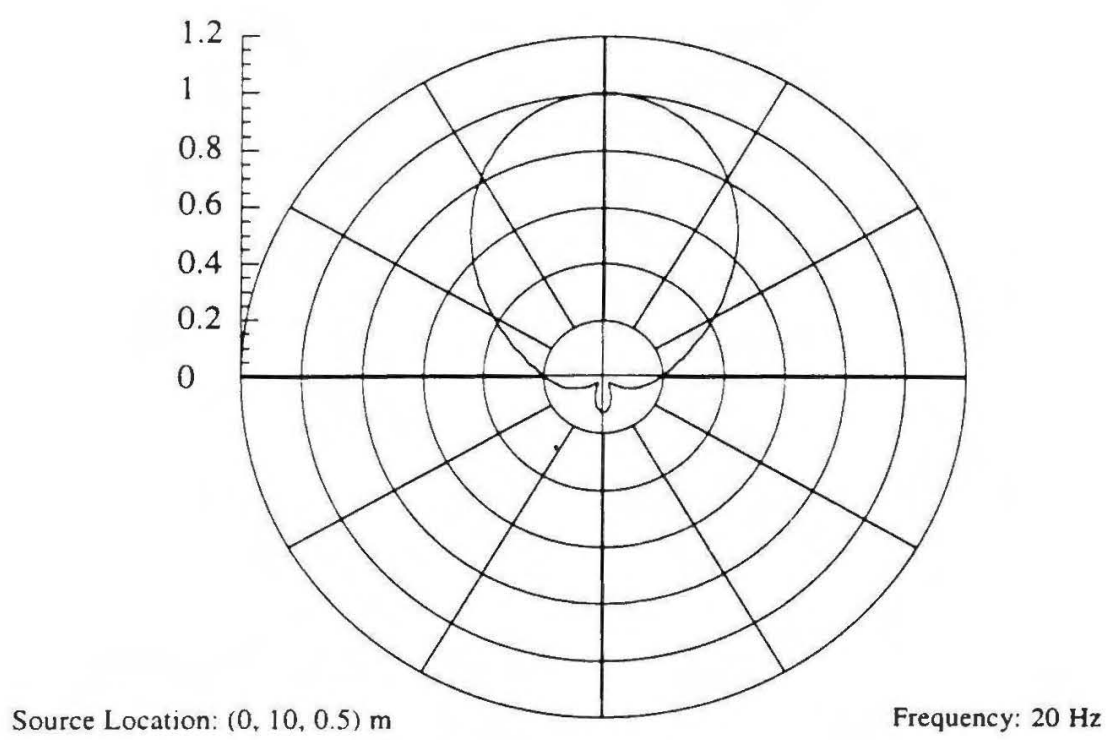

Figure 2.10 The MOM simulation of the shed
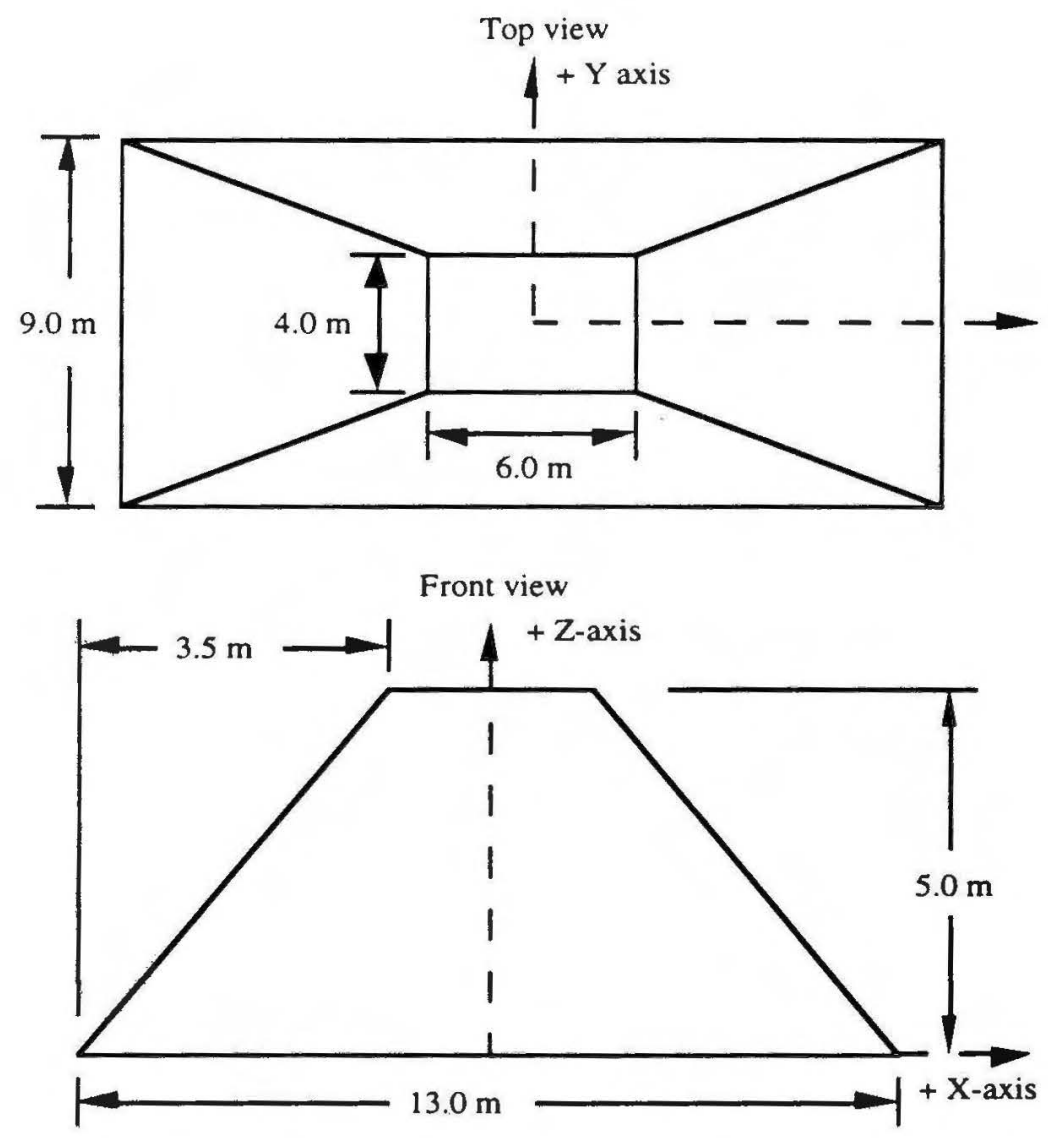

Figure 2.11 The hill 


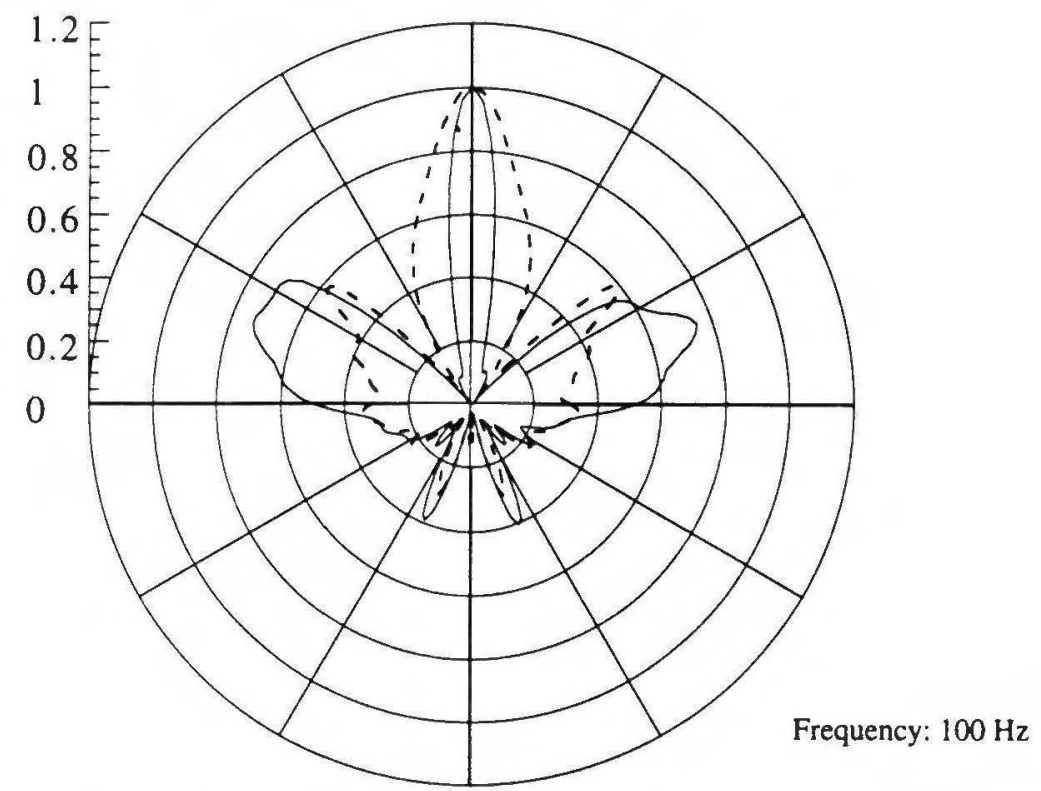

Figure 2.12 The MOM simulations of the hill

\subsection{Problems In MOM}

Although Alona Boag's program accurately predicts the scattering pattern, there are several difficulties in using her codes. First of all, the mesh generator she used is not userfriendly. For example, to mesh the rectangular baffle, one has to enter four vertices coordinates for each rectangular face of the baffle counter clockwise, looking from the outside of the baffle. This ensures the correct calculation of outward normal vectors. It is simple for the baffle with six faces, but when a geometry is complex and has many facets, it is tedious to enter the coordinates manually. In addition, an accurate meshing of any curved surface is impossible since the mesh generator assumes a flat surface. To create geometry and mesh its surfaces with ease, a finite element analysis package called PATRAN is explored. A few simple PATRAN commands create geometry, mesh surfaces, and output the 
coordinates. Creating and meshing curvatures are simple with PATRAN. However, there is a drawback in PATRAN. To define outward normal vectors on surfaces of an object, the coordinates should be listed in counter clockwise sequence. PATRAN meshes and lists element coordinates arbitrarily, either clockwise or counter clockwise. Some normal vectors point outward, and others inward. To remove this arbitrariness, a simple routine is written. Assuming each element is approximately equal in size, the center coordinates of each element are summed up and then divided by the number of elements, $\mathrm{N}$. This yields an approximate center coordinate of the scatterer, and a dot product of a vector from this center of the object to a center of any element and the corresponding normal vector should be a positive quantity. If negative, then it indicates the normal vector is pointing toward the center of the object, and the direction of the vector should be reversed.

Other difficulties are the memory and CPU time needed in computing. Since the number of elements $\mathrm{N}$ is proportional to the surface area, analysis of an object large compared to the wavelength is difficult, if not impossible. As $\mathrm{N}$ increases the required memory for storing the impedance matrix increases as $\mathrm{N}^{2}$. In FORTRAN, reserving a large, contiguous section of working memory becomes difficult when solving for a large number of unknowns. For example, in the Michigan baffle simulation at $163 \mathrm{~Hz}, 7,308$ triangle elements are meshed, and the number of the matrix elements is $53,406,864$. Each matrix element occupies 16 bytes of memory for a complex double precision data type. Thus more than 854 MBytes are needed in creating the matrix. The CPU time for a matrix inversion is proportional to $\mathrm{N}^{3}$. Most of the acoustic scattering simulations have been solved in a Convex C420 machine at the University of Illinois. The Convex C420 has four processors with 512 MBytes of memory. It is used for high speed, heavily vectorized computations. However $\mathrm{C} 420$ has proved to be inadequate for the baffle simulation at $163 \mathrm{~Hz}$. An access to a more powerful machine is obtained from National Center for Supercomputing Applications (NCSA): Power Challenge account. The Power Challenge Machine is built by Silicon Graphics, Inc., and it has 16 shared memory multiprocessors. Its total memory is four giga bytes, and it can perform 300 mega floating point operations per second (flops) per 
processor, total of 4.8 giga flops.

In spite of the increased capacity, a more powerful machine is needed in analyzing larger objects at high frequencies. The limited resources are unavoidable. One should look for a clever method of solving the problem instead of looking for a faster machine. A technique that overcomes all of the above problems, meshing, memory, and CPU time, is sought and implemented in Chapter 3. 


\section{CHAPTER 3 \\ BODY OF REVOLUTION}

As discussed in Chapter 2, MOM has many limitations in analyzing large, curved scatterers. A common example of such a scatterer is a parabolic reflector. A parabolic reflector is axisymmetric, so a method called body of revolution (BOR) can be used to solve such a case. The study of the BOR technique is based on a paper by Seybert et al. [6]. By taking an advantage of the axisymmetric properties, the surface integral is reduced to a line integral along the generator of the scatterer body and an integral over the angle of the revolution. The integration over the angle is performed partly analytically in terms of elliptic integrals and partly numerically using the Gaussian quadrature formula. A program in FORTRAN is written, and the same test cases in the Seybert's paper are run and compared to the paper. My results agree well with both the paper and the theoretical solution. This chapter describes BOR in detail.

\subsection{Derivation Of BOR}

The derivation of BOR is based on the Helmholtz integral formula,

$$
\mathrm{C}(\mathbf{r}) \psi(\mathbf{r})=\int_{\mathrm{s}} \psi\left(\mathbf{r}^{\prime}\right) \frac{\partial \mathrm{G}\left(\mathbf{r}, \mathbf{r}^{\prime}\right)}{\partial \mathrm{n}} \mathrm{ds}+4 \pi \psi^{\mathrm{inc}}(\mathbf{r})
$$

where

$$
C(\mathbf{r})=4 \pi+\int_{s} \frac{\partial}{\partial n}\left(\frac{1}{R\left(\mathbf{r}, \mathbf{r}^{\prime}\right)}\right) d s\left(\mathbf{r}^{\prime}\right)
$$

Throughout the derivation of BOR, the time dependence of $\exp (i \omega t)$ is used, and the Green's function is taken as $G\left(\mathbf{r}, \mathbf{r}^{\prime}\right)=\exp (-i k R) / R$ to follow the notations of the Seybert paper. The integral in (3.2) approaches $-2 \pi$ on the smooth surface of the body.

Figure 3.1 illustrates a simple axisymmetric scatterer and the nomenclature needed in the derivation of BOR. When the scatterer, boundary conditions, and sources are 
axisymmetric, the pressure fields and the normal velocity fields on the surface of the scatterer are also axisymmetric. Taking an advantage of this symmetric distribution of the fields, the surface integral may be reduced to a line integral.

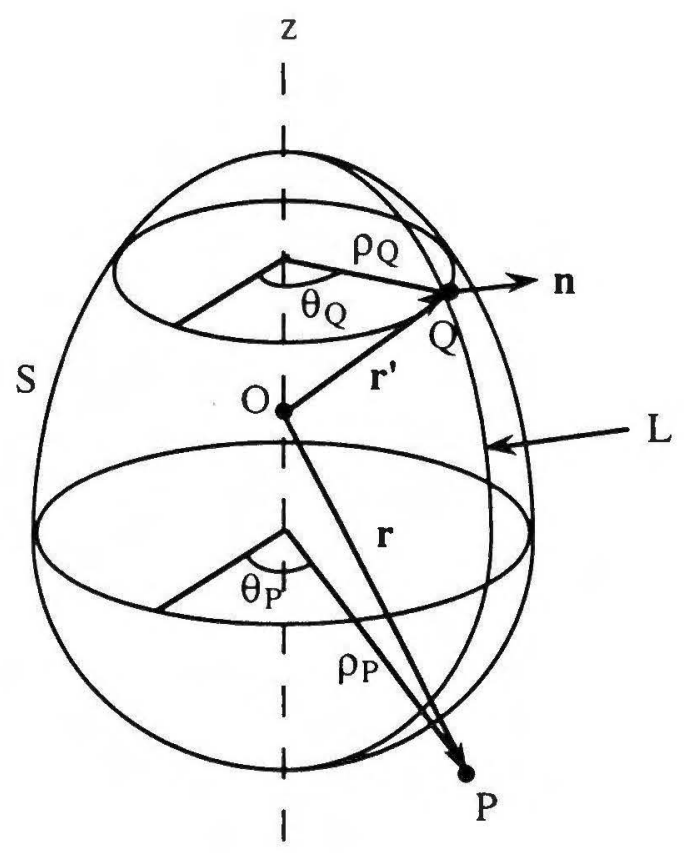

Figure 3.1 A simple axisymmetric scatterer

Because the axisymmetric body is independent of the angle of revolution $\theta, \psi(\rho, \theta, z)$ depends only on $z$ and $\rho$. The integral in (3.1) may be expressed as below:

$$
\begin{aligned}
& \int_{L} \psi\left(\mathbf{r}^{\prime}\right)\left[\int_{0}^{2 \pi} \frac{\partial}{\partial n}\left(\frac{\exp \left(-i k R\left(\mathbf{r}, \mathbf{r}^{\prime}\right)\right.}{R\left(\mathbf{r}, \mathbf{r}^{\prime}\right)}\right) \mathrm{d} \theta\left(\mathbf{r}^{\prime}\right)\right] \rho\left(\mathbf{r}^{\prime}\right) \mathrm{dL}\left(\mathbf{r}^{\prime}\right) \\
& \quad-\int_{L} \frac{\partial \psi\left(\mathbf{r}^{\prime}\right)}{\partial \mathrm{n}}\left(\int_{0}^{2 \pi} \frac{\exp \left(-i k R\left(\mathbf{r}, \mathbf{r}^{\prime}\right)\right.}{R\left(\mathbf{r}, \mathbf{r}^{\prime}\right)} \mathrm{d} \theta\left(\mathbf{r}^{\prime}\right)\right) \rho\left(\mathbf{r}^{\prime}\right) \mathrm{dL}\left(\mathbf{r}^{\prime}\right)
\end{aligned}
$$

For convenience, let

$$
\mathrm{K}\left(\mathbf{r}, \mathbf{r}^{\prime}\right)=\int_{0}^{2 \pi} \frac{\partial}{\partial \mathrm{n}}\left(\frac{\exp \left(-\mathrm{ikR}\left(\mathbf{r}, \mathbf{r}^{\prime}\right)\right.}{\mathrm{R}\left(\mathbf{r}, \mathbf{r}^{\prime}\right)}\right) \mathrm{d} \theta\left(\mathbf{r}^{\prime}\right)
$$


The above equation can be decomposed into two parts,

$$
\begin{gathered}
\mathrm{K}_{1}\left(\mathbf{r}, \mathbf{r}^{\prime}\right)=\int_{0}^{2 \pi} \frac{\partial}{\partial \mathrm{n}}\left(\frac{\exp \left(-\mathrm{ikR}\left(\mathbf{r}, \mathbf{r}^{\prime}\right)-1\right.}{\mathrm{R}\left(\mathbf{r}, \mathbf{r}^{\prime}\right)}\right) \mathrm{d} \theta\left(\mathbf{r}^{\prime}\right) \\
\mathbf{K}_{2}\left(\mathbf{r}, \mathbf{r}^{\prime}\right)=\int_{0}^{2 \pi} \frac{\partial}{\partial \mathrm{n}}\left(\frac{1}{R\left(\mathbf{r}, \mathbf{r}^{\prime}\right)}\right) \mathrm{d} \theta\left(\mathbf{r}^{\prime}\right)
\end{gathered}
$$

As $R$ approaches zero, the integrand of $K_{1}\left(\mathbf{r}, \mathbf{r}^{\prime}\right)$ can be expressed in terms of a Taylor's series expansion,

$$
\frac{\exp \left(-\mathrm{ikR}\left(\mathbf{r}, \mathbf{r}^{\prime}\right)-1\right.}{\mathrm{R}\left(\mathbf{r}, \mathbf{r}^{\prime}\right)} \approx \frac{1-\mathrm{ikR}\left(\mathbf{r}, \mathbf{r}^{\prime}\right)+0.5\left(-\mathrm{ikR}\left(\mathbf{r}, \mathbf{r}^{\prime}\right)\right)^{2}-1}{R\left(\mathbf{r}, \mathbf{r}^{\prime}\right)}=-\mathrm{ik}-\frac{\mathrm{k}^{2} \mathbf{R}\left(\mathbf{r}, \mathbf{r}^{\prime}\right)}{2}
$$

Let the normal component of $R\left(\mathbf{r}, \mathbf{r}^{\prime}\right)$ be $d$, and the tangential component be $\mathrm{x}$. Then the derivative of (3.7) becomes,

$$
\frac{\partial}{\partial n}\left(-i k-\frac{k^{2} R\left(\mathbf{r}, \mathbf{r}^{\prime}\right)}{2}\right)=-\frac{k^{2}}{2} \frac{\partial R\left(\mathbf{r}, \mathbf{r}^{\prime}\right)}{\partial n}=-\frac{k^{2}}{2} \frac{d}{\sqrt{x^{2}+d^{2}}}
$$

As $x$ approaches zero, (3.8) will be $-\frac{\mathrm{k}^{2}}{2}$, so $\mathrm{K}_{1}\left(\mathbf{r}, \mathbf{r}^{\prime}\right)$ is non-singular and can be evaluated numerically at $R\left(\mathbf{r}, \mathbf{r}^{\prime}\right)=0 . K_{2}\left(\mathbf{r}, \mathbf{r}^{\prime}\right)$ can be expressed in terms of the elliptic integrals.

$$
K_{2}\left(\mathbf{r}, \mathbf{r}^{\prime}\right)=\frac{\partial}{\partial \mathrm{n}}\left[\int_{0}^{2 \pi} \frac{1}{\mathrm{R}\left(\mathbf{r}, \mathbf{r}^{\prime}\right)} \mathrm{d} \theta\left(\mathbf{r}^{\prime}\right)\right]=\frac{\partial}{\partial \mathrm{n}}\left[\frac{4}{\overline{\mathrm{R}}} \int_{0}^{2 \pi} \frac{\mathrm{d} \varphi}{\left(1-\overrightarrow{\mathrm{k}}^{2} \sin ^{2} \varphi\right)^{1 / 2}}\right]=\frac{\partial}{\partial \mathrm{n}}\left[\frac{4}{\overline{\mathrm{R}}} \mathrm{F}(\pi / 2, \overline{\mathrm{k}})\right]
$$

where $F(\pi / 2, \bar{k})$ is a complete elliptic integral of the first kind with modulus $\bar{k}$. Also, $\overline{\mathrm{R}}^{2}=\left(\rho_{\mathrm{Q}}+\rho_{\mathrm{P}}\right)^{2}+\left(\mathrm{z}_{\mathrm{Q}}+\mathrm{z}_{\mathrm{P}}\right)^{2}$ and $\overline{\mathrm{k}}=\frac{4 \rho_{\mathrm{Q}} \rho_{\mathrm{P}}}{\overline{\mathrm{R}}^{2}}$. The outward normal vector at point $\mathrm{Q}$ is defined as $\hat{n}=n_{\rho Q} \hat{\rho}+n_{z Q} \hat{z}$, and the angle of revolution is $\theta=\theta_{Q}-\theta_{P}$. Evaluating (3.9) yields,

$$
\mathrm{K}_{2}\left(\mathbf{r}, \mathbf{r}^{\prime}\right)=\frac{4}{\overline{\mathrm{R}}^{2}}\left[\left(\overline{\mathrm{R}} \frac{\partial \mathrm{F}}{\partial \overline{\mathrm{k}}} \frac{\partial \overline{\mathrm{k}}}{\partial \rho_{\mathrm{Q}}}-\mathrm{F} \frac{\partial \overline{\mathrm{R}}}{\partial \rho_{\mathrm{Q}}}\right) \mathrm{n}_{\rho \mathrm{Q}}+\left(\overline{\mathrm{R}} \frac{\partial \mathrm{F}}{\partial \overline{\mathrm{k}}} \frac{\partial \overline{\mathrm{k}}}{\partial \mathrm{z}_{\mathrm{Q}}}-\mathrm{F} \frac{\partial \overline{\mathrm{R}}}{\partial \mathrm{z}_{\mathrm{Q}}}\right) \mathrm{n}_{\mathrm{zQ}}\right]
$$

The derivative of $\mathrm{F}$ is expressed in terms of the complete elliptic integral of the second kind, 


$$
\frac{\partial \mathrm{F}}{\partial \overline{\mathrm{k}}}=\frac{\mathrm{E}-\overline{\mathrm{k}}^{2} \mathrm{~F}}{\overline{\mathrm{k}} \overline{\mathrm{k}}^{2}}
$$

where $E=\int_{0}^{2 \pi}\left(1-\overline{\mathrm{k}}^{2} \sin ^{2} \varphi\right)^{1 / 2} \mathrm{~d} \varphi$ and $\overline{\mathrm{k}^{\prime}}=\left(1-\overline{\mathrm{k}}^{2}\right)^{1 / 2}$. Substituting above expressions and evaluating derivatives in (3.10),

$$
\mathrm{K}_{2}\left(\mathbf{r}, \mathbf{r}^{\prime}\right)=\frac{4}{\overline{\mathrm{R}}^{2}}\left[\left(\frac{1}{2} \frac{\overline{\mathrm{R}} \mathrm{E}}{\rho_{\mathrm{Q}} \overline{\mathrm{k}^{\prime 2}}}-\frac{1}{2} \frac{\overline{\mathrm{R}} \mathrm{F}}{\rho_{\mathrm{Q}}}-\frac{\mathrm{E}\left(\rho_{\mathrm{Q}}+\rho_{\mathrm{Q}}\right)}{\overline{\mathrm{R}} \overline{\mathrm{k}^{\prime 2}}}\right) \mathrm{n}_{\rho_{\mathrm{Q}}}-\frac{\mathrm{E}\left(\mathrm{z}_{\mathrm{Q}}-\mathrm{z}_{\mathrm{P}}\right)}{\overline{\mathrm{k}^{\prime 2}} \overline{\mathrm{R}}} \mathrm{n}_{\mathrm{zQ}}\right]
$$

Thus the surface integral in (3.1) is expressed as a line integral as below:

$$
\mathrm{C}(\mathbf{r}) \psi(\mathbf{r})=\int_{L} \psi\left(\mathbf{r}^{\prime}\right) \mathrm{K}\left(\mathbf{r}, \mathbf{r}^{\prime}\right) \rho\left(\mathbf{r}^{\prime}\right) \mathrm{dL}\left(\mathbf{r}^{\prime}\right)+4 \pi \psi^{\mathrm{inc}}(\mathbf{r})
$$

where $C(\mathbf{r})=4 \pi+\int_{L} K_{2}\left(\mathbf{r}, \mathbf{r}^{\prime}\right) \rho\left(\mathbf{r}^{\prime}\right) \mathrm{dL}\left(\mathbf{r}^{\prime}\right)$.

\subsection{Numerical Implementation}

Equation (3.13) can be numerically evaluated along a contour $L$ of an axisymmetric body. The line $\mathrm{L}$ is divided into segments for integration (see Figure 3.2).

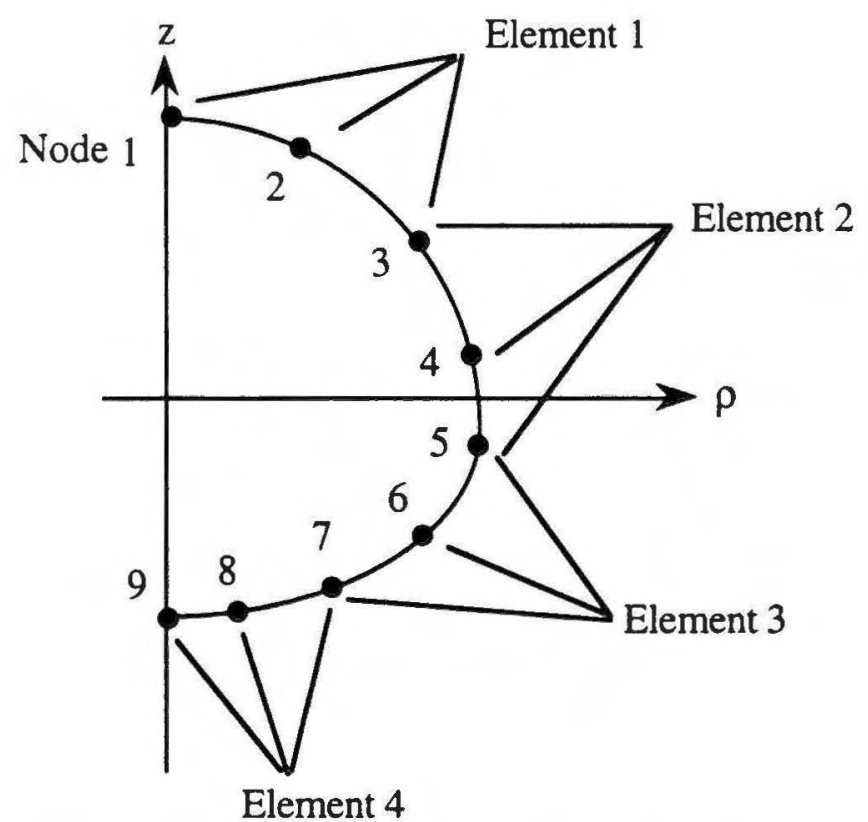

Figure 3.2 Discretization on the contour $\mathrm{L}$ 
The line $\mathrm{L}$ is divided into four elements where each element is made up of three nodes. The contour along the line $\mathrm{L}$ is assumed to be varying quadratically, and the variation is called a shape function, $\mathrm{N}_{\alpha}(\xi)$. A quadratic shape function is defined as below, and it provides a more accurate description of the contour than does a linear shape function. $\xi$ is called a local coordinate and ranges from -1 to 1 , and $\alpha$ is called a local node number and ranges from 1 to 3.

$$
\begin{array}{ll}
\mathrm{N}_{\alpha}(\xi)=-\frac{1}{2} \xi+\frac{1}{2} \xi^{2} & , \alpha=1 \\
\mathrm{~N}_{\alpha}(\xi)=1-\xi^{2} & , \alpha=2 \\
\mathrm{~N}_{\alpha}(\xi)=\frac{1}{2} \xi+\frac{1}{2} \xi^{2} & , \alpha=3
\end{array}
$$

The actual coordinates can be represented in terms of the shape function and local coordinate,

$$
\begin{aligned}
& \rho(\xi)=\sum_{\alpha=1}^{3} N_{\alpha}(\xi) \rho_{\alpha} \\
& z(\xi)=\sum_{\alpha=1}^{3} N_{\alpha}(\xi) z_{\alpha}
\end{aligned}
$$

The above equations map each element on the body onto a straight line in the local coordinate $\xi$.

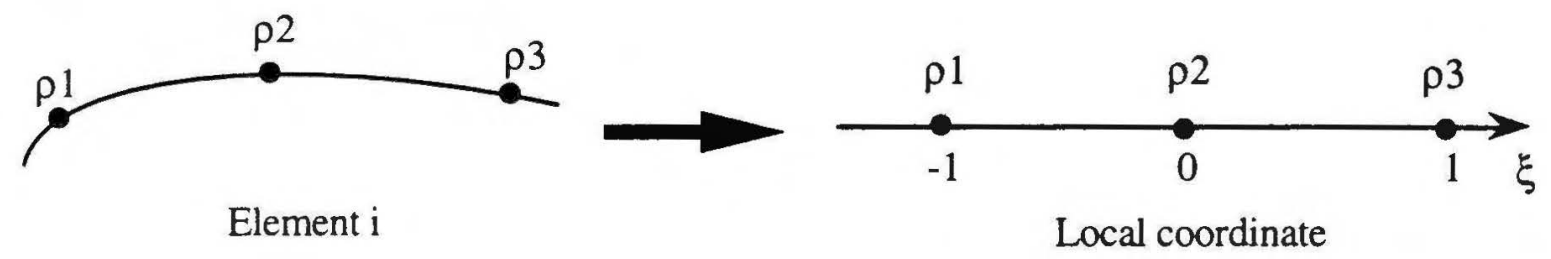

Figure 3.3 The element mapping onto the local coordinate system

Similarly the pressure fields $\psi$ along $L$ are approximated using the same shape function.

$$
\psi_{\mathrm{m}}(\xi)=\sum_{\alpha=1}^{3} \mathrm{~N}_{\alpha}(\xi) \psi_{\mathrm{m} \alpha}
$$

where $\psi_{\mathrm{m} \alpha}$ is the value at node $\alpha$ on the element $\mathrm{m}$. Because the fields distribution and the contour are described using the same shape function, the formulation is said to be isoparametric. 
Applying the above scheme to the line integral Equation (3.13) yields,

$$
C(\mathbf{r}) \psi(\mathbf{r})=\sum_{m=1}^{N} \sum_{\alpha=1}^{3} \psi_{m} \int_{-1}^{1} K\left(\mathbf{r}, \mathbf{r}^{\prime}(\xi)\right) N_{\alpha}(\xi) \rho(\xi) J_{m}(\xi) d \xi+4 \pi \psi^{\text {inc }}(\mathbf{r})
$$

where

$$
\mathrm{C}(\mathbf{r})=4 \pi+\sum_{\mathrm{m}=1}^{3} \int_{-1}^{1} K_{2}\left(\mathbf{r}, \mathbf{r}^{\prime}(\xi)\right) \rho(\xi) J_{m}(\xi) d \xi
$$

$\mathrm{N}$ is the total number of elements used to discretize the scatterer body, and $\mathrm{J}_{\mathrm{m}}(\xi)$ is the Jacobian of transformation for element $m$.

$$
\begin{gathered}
J_{m}(\xi)=\left[\left(\frac{\partial \rho}{\partial \xi}\right)^{2}+\left(\frac{\partial z}{\partial \xi}\right)^{2}\right]^{1 / 2}= \\
{\left[\frac{1}{2}(\rho 3-\rho 1) \xi+(\rho 1+\rho 3-2 \rho 2)^{2}+\frac{1}{2}(z 3-z 1) \xi+(z 1+z 3-2 z 2)^{2}\right]^{1 / 2}}
\end{gathered}
$$

Therefore for any point $P$ on the surface of the scatterer, whose global node number is $\mathrm{j}$, the integral equation can be expressed as follow:

$$
-\sum_{m=1}^{3} \sum_{\alpha=1}^{3} \psi_{m \alpha} a_{m j}^{\alpha}+\psi_{j}\left(1+\sum_{m=1}^{N} C_{m j}\right)=\psi_{j}^{i n c}
$$

where

and

$$
\begin{gathered}
a_{m j}^{\alpha}=\frac{1}{4 \pi} \int_{-1}^{1} K\left(P_{j}, \xi\right) N_{\alpha}(\xi) \rho(\xi) J_{m}(\xi) d \xi \\
C_{m j}=\frac{1}{4 \pi} \int_{-1}^{1} K_{2}\left(P_{j}, \xi\right) \rho(\xi) J_{m}(\xi) d \xi
\end{gathered}
$$

Equations (3.20) to (3.22) apply to each node, and those $\mathrm{M}$ equations make up a matrix equation for BOR.

$$
[\mathrm{A}][\psi]=\left[\psi^{\mathrm{inc}}\right]
$$

For integration on the $\xi$ axis, the Gaussian quadrature was used. Initially a 4-point Gaussian quadrature formula was used to evaluate the integral. Comparing with the theoretical solution 
revealed that the 4-point Gaussian quadrature introduced 20 - 30\% error. 6- or 8-point Gaussian quadrature must be used for an accurate numerical integration. In addition the number of total nodes were varied, and the number of nodes did not contribute much to the accurate analysis. For example, scattered patterns from 9, 17, and 33 node-bodies were similar to one another. Thus beyond a reasonable number of nodes, the number of total nodes will lengthen the computational time without any improvement on the accuracy.

\subsection{Scattering From Spheres}

Several cases were run to verify the validity of the code. To compare with the results from the reference paper [6], identical test parameters were chosen. Seybert et al presented the scattered pressure field patterns of spheres due to an incident plane wave [6]. The test case results closely agree with the results from the paper. In addition, a theoretical scattering pattern for Figure 3.4 was computed in Mathematica for a further comparison. Due to its simple geometry, scattering by a sphere can be solved analytically. For a rigid sphere with a plane wave incident in $-\mathrm{z}$ axis, the total field is [7],

$$
\psi^{\text {tot }}=\psi^{i n c}+\psi^{s}=\sum_{n=0}^{\infty}(-i)^{n}(2 n+1)\left[j_{n}(k r)-a_{n}^{\prime} h_{n}^{(1)}(k r)\right] P_{n}(\cos \theta)
$$

where $j_{n}$ and $h_{n}^{(1)}$ are spherical Bessel and Hankel functions, respectively. Also $a_{n}^{\prime}=\frac{j_{n}^{\prime}(k a)}{h_{n}^{(1)^{\prime}}(k a)}$ where $\mathrm{a}$ is the radius of the sphere. $\mathrm{P}_{\mathrm{n}}$ is the associated Legendre function. Since the incident field is $\exp (-\mathrm{ikr} \cos \theta)$, the scattered field is $\psi^{\mathrm{tot}}-\exp (-\mathrm{ikr} \cos \theta)$. In the actual calculation of the analytic solution, the summation in (3.24) were added up to $n=30$ (see Figure 3.5). The test cases involving only one scattering body were compared to the analytic solutions, and both my results and [6] closely followed the analytic solutions (see Figure 3.6). For test cases involving two discrete spheres, approximate solutions, which neglected the interactions between the two bodies, were compared to the numerical solutions (see Figures $3.7,3.8$, and 3.9). 


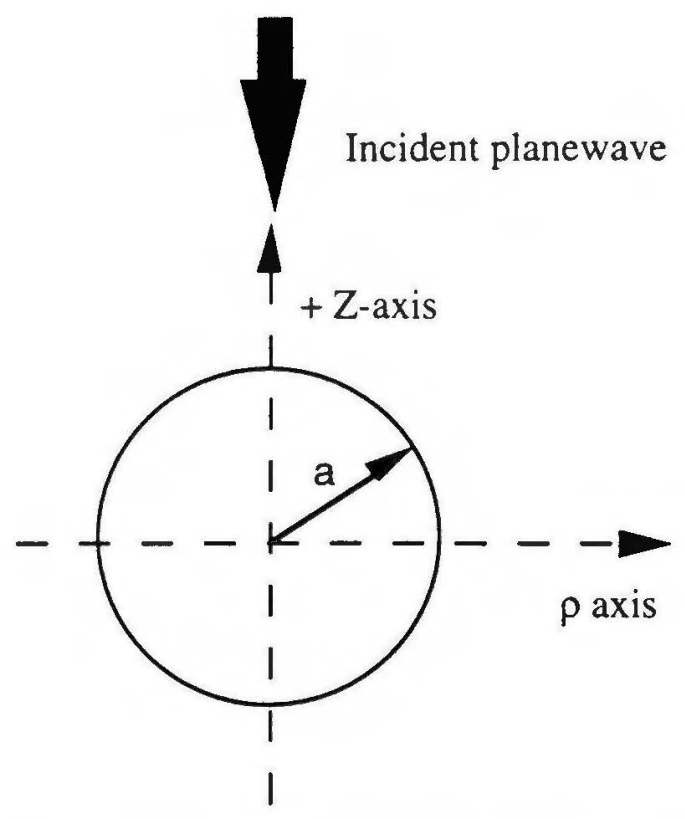

Figure 3.4 Scattering from one sphere

Scattered pressure field amplitude in the azimuthal plane

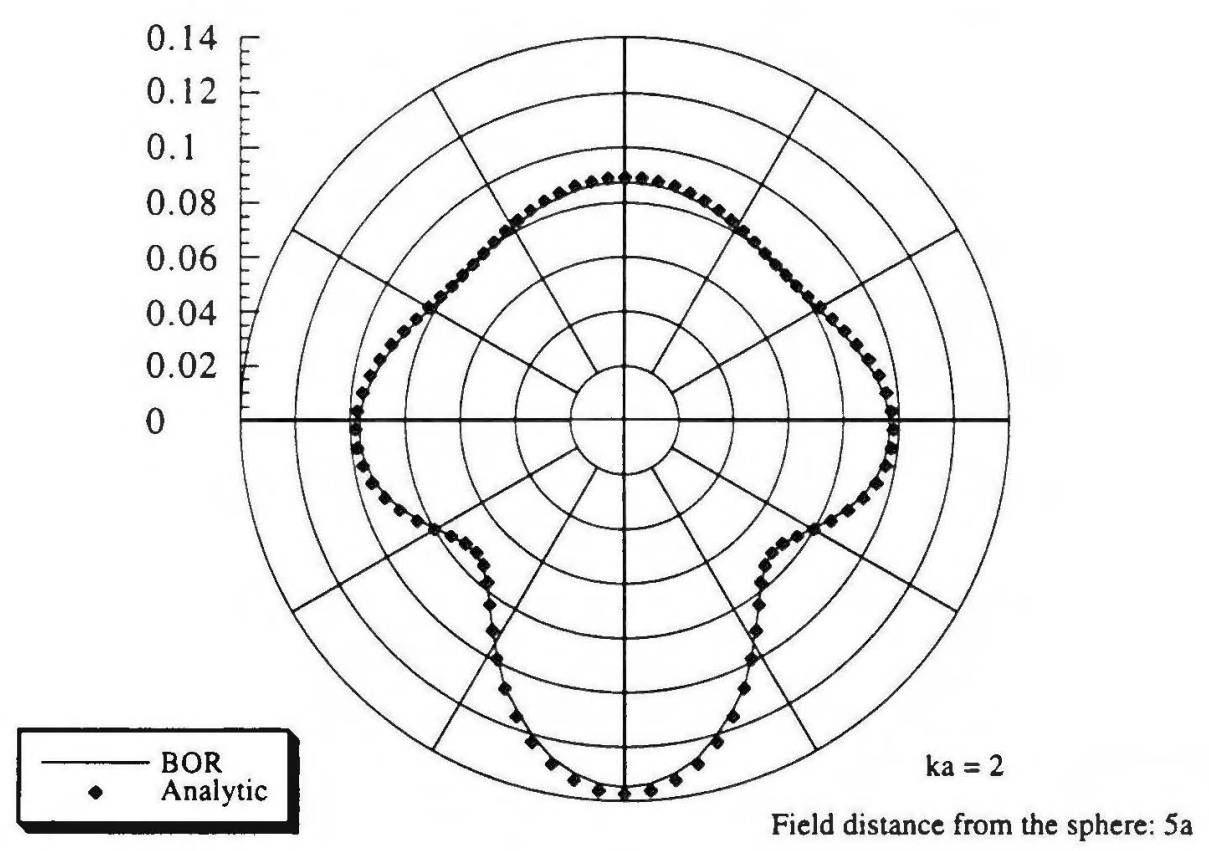

Figure 3.5 The BOR simulation and analytic scattering patterns of the sphere 
Scattered pressure field amplitude in the azimuthal plane

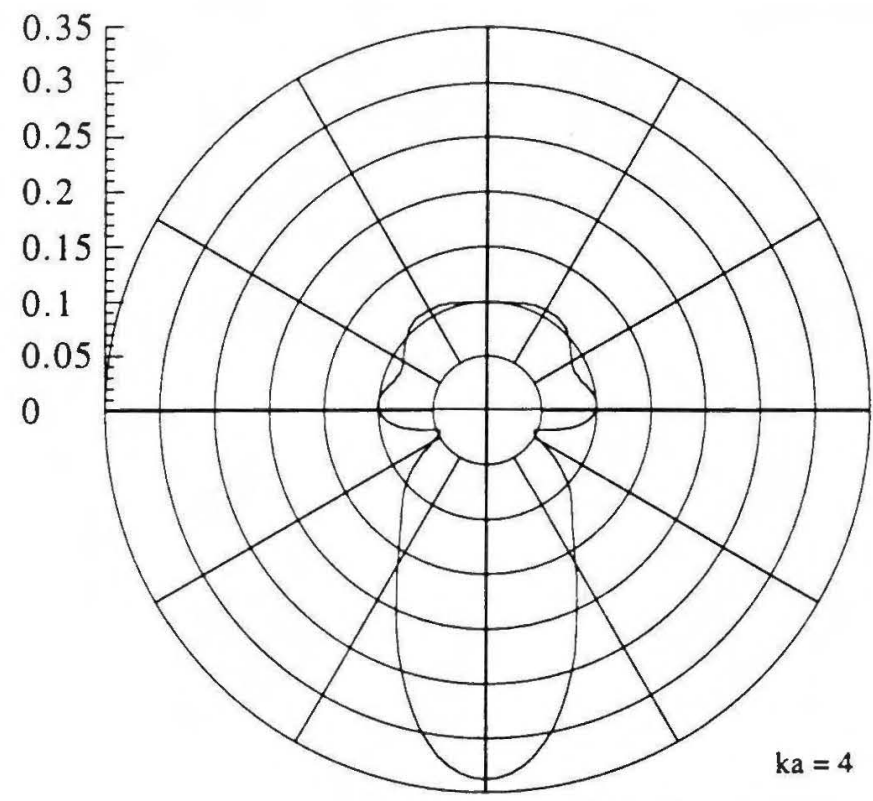

Field distance from the sphere: $5 \mathrm{a}$

Figure 3.6 The BOR simulation of the sphere

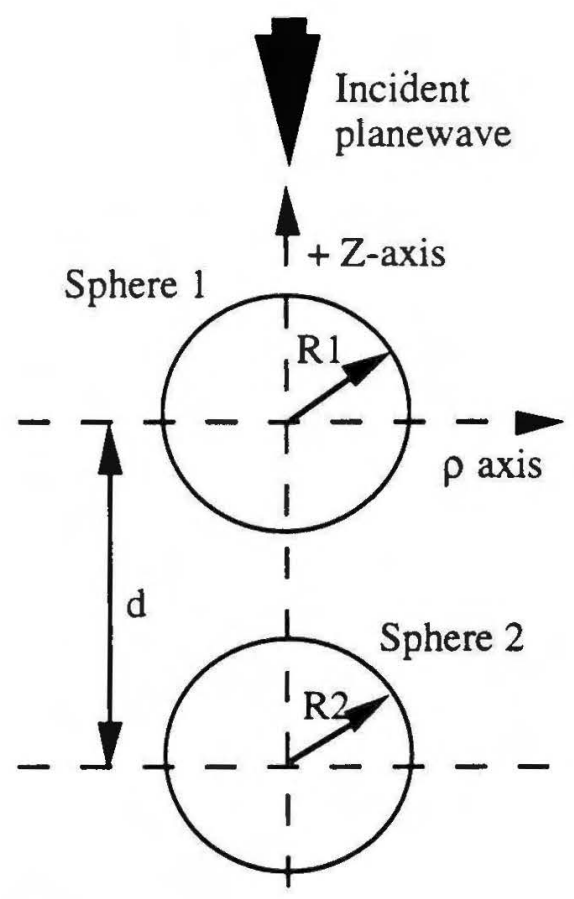

Figure 3.7 Scattering from two spheres 
Scattered pressure field amplitude in the azimuthal plane

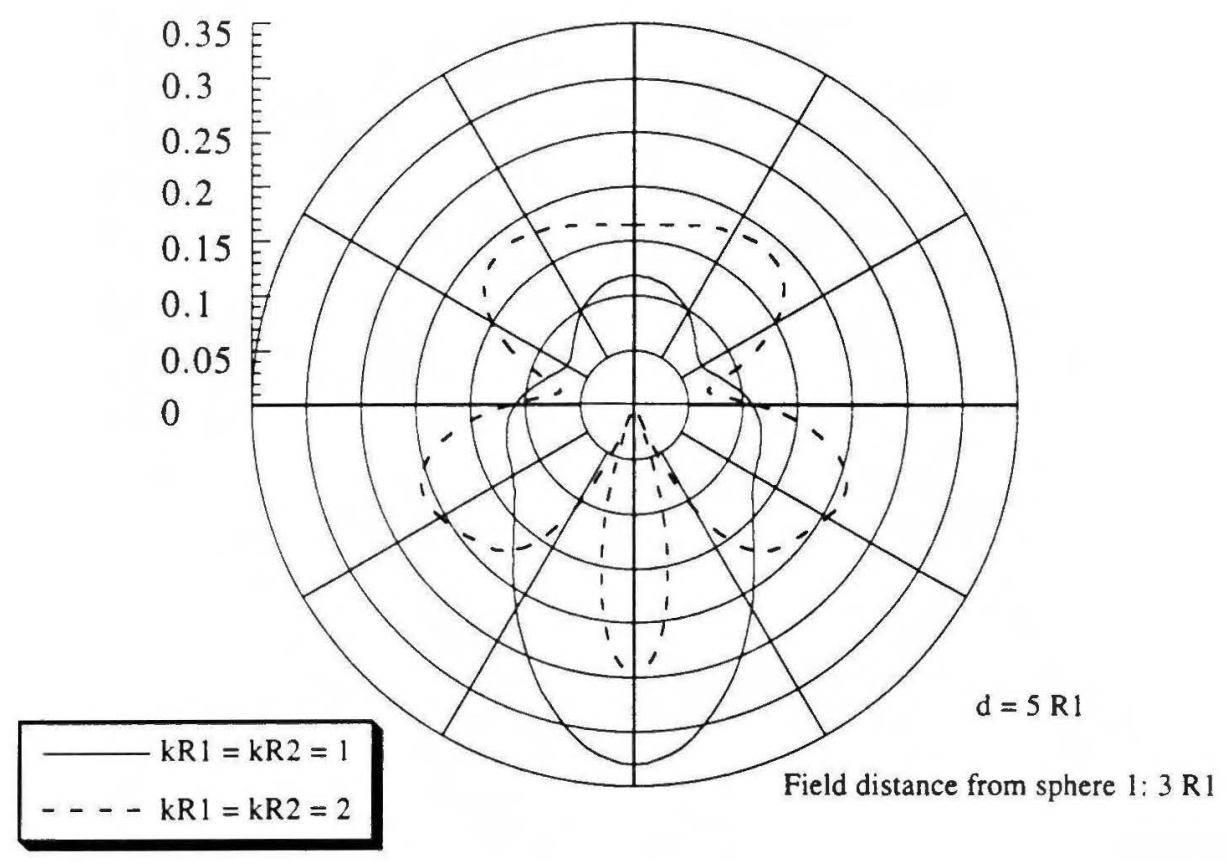

Figure 3.8 The BOR simulations of the two spheres 1

Scattered pressure field amplitude in the azimuthal plane

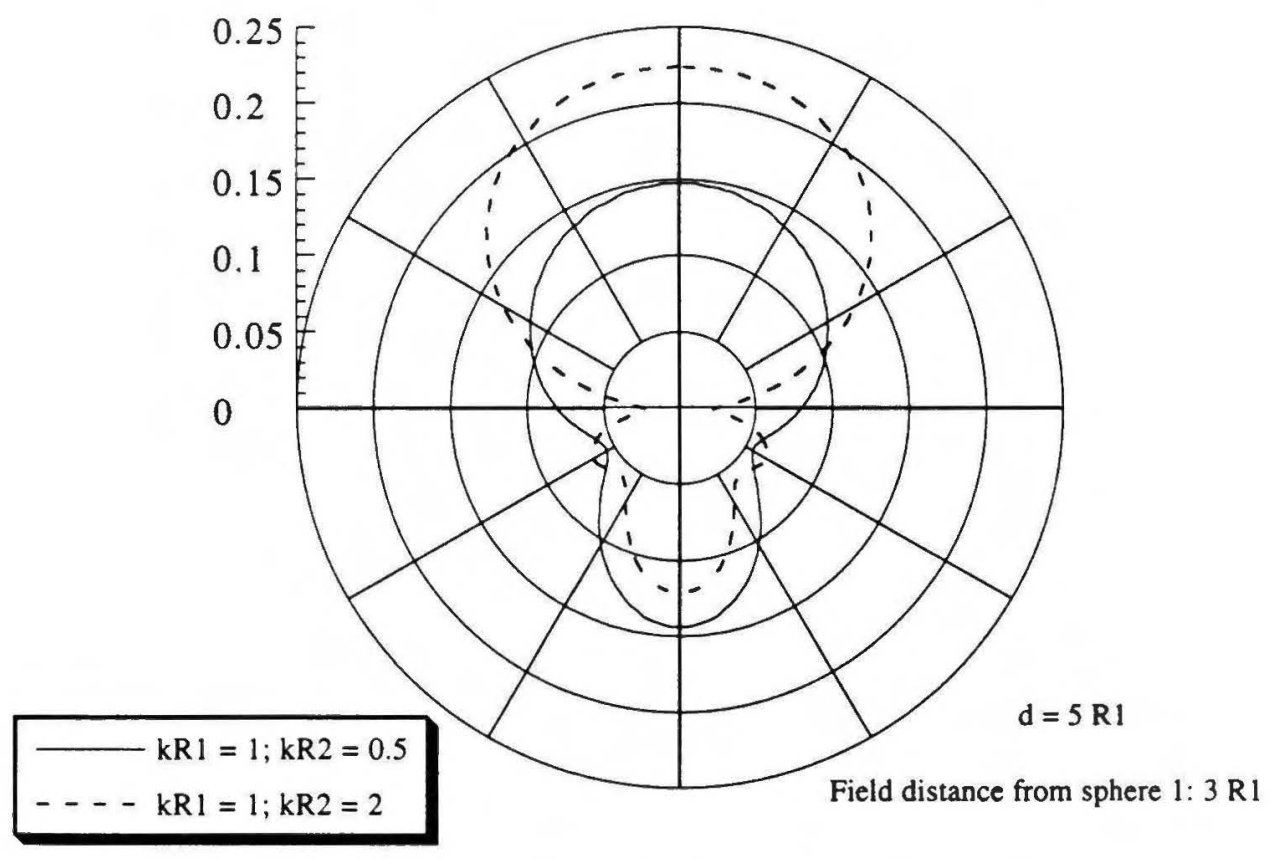

Figure 3.9 The BOR simulations of the two spheres 2 


\subsection{Scattering From Parabolic Reflectors}

Comparing the simulation results in Section 3.3 is not adequate for a complete validation of the BOR code. Scattering from the spheres is axisymmetric regardless of the propagation direction of the incident field. Also only the diffractions due to a plane wave incidence have been compared and validated. For a complete testing of the BOR code, scattering due to a point source incident on an object such as a parabolic reflector should be tested.

Simulations on the diffractions of the parabolic reflectors can be useful since the parabolic reflectors are used as acoustic amplifiers [8]. Recording bird sounds in the wilderness is not a trivial task for ornithologists since the bird sounds may not be loud enough to be distinguished in the presence of background noises. Many ornithologists use parabolic microphones to record bird sounds. A parabolic microphone is a parabolic reflector dish with a microphone attached to the dish. Typically the microphone is located at the focal point of the parabolic reflector because the fields will arrive in equi-phase at the surface of the reflector. Using the reciprocity theorem, a diffraction pattern due to a point source at a focal point is identical to the reception pattern of the microphone located at the focal point.

Although the use of the parabolic microphones are common in bird recordings, exact reception patterns are not supplied by their manufacturers. The receptivity of parabolic reflectors is thought to be highly directional. Manufacturing parabolic microphones is not as simple as building flat baffle-type directional microphones because of the curvature. Thus the price of the parabolic microphones are quite expensive. The diffraction patterns from various parabolic reflectors can be accurately calculated by the BOR code. Note that as the focal point is place farther away from the reflector, the curvature of the reflector becomes more flat (see Figure 3.10). Figures 3.11 through 3.15 show far field diffraction patterns for parabolic reflectors whose diameters are twice the acoustic wavelength of the interest. The reflector analyzed in Figure 3.15 is virtually a disk since the focal point is 100 wavelengths away from the center of the reflector. Except for Figure 3.11, the highest directivity is achieved when the point source is placed at 0.5 wavelength away from the center. This is plausible 
because the scattered fields on the surface of the reflectors have the same phase as the incident fields on the rigid reflectors. Therefore when the source is positioned at 0.5 wavelength away from the center, the incident and reflected fields are also in-phase, adding up the field amplitudes. The patterns show that the parabolic reflectors do not neccessarily achieve high directivity. Large side lobes can be found depending on the positions of the source. Figure 3.16 compares the diffraction patterns of a parabolic reflector, disk, and a rectangular baffle with similar dimensions. The parabolic reflector achieves slightly higher directivity, and it is not enough to justify for purchasing expensive parabolic reflector microphones.

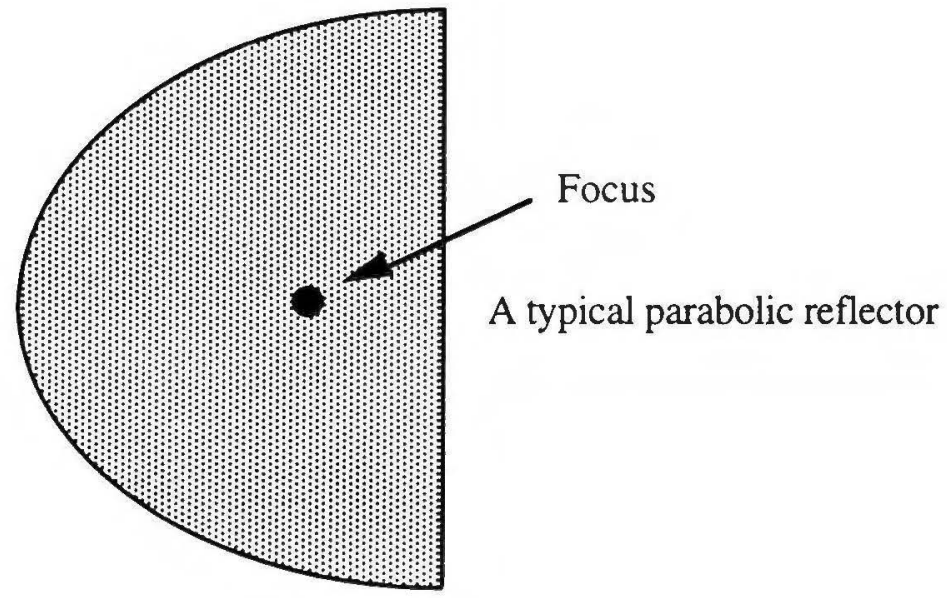

A disk-like parabolic reflector

Focus

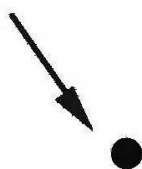

Figure 3.10 The parabolic reflectors 
Total pressure field amplitude in the azimuthal plane

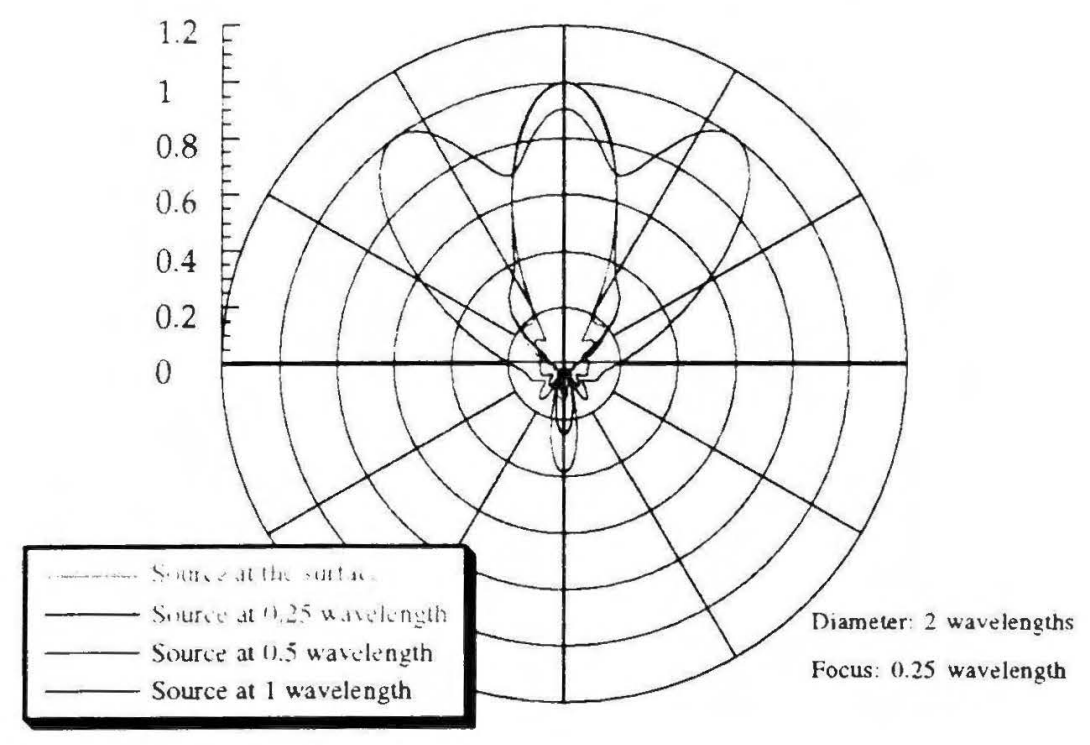

Figure 3.11 The BOR simulations of the parabolic reflector 1

Total pressure field amplitude in the azimuthal plane

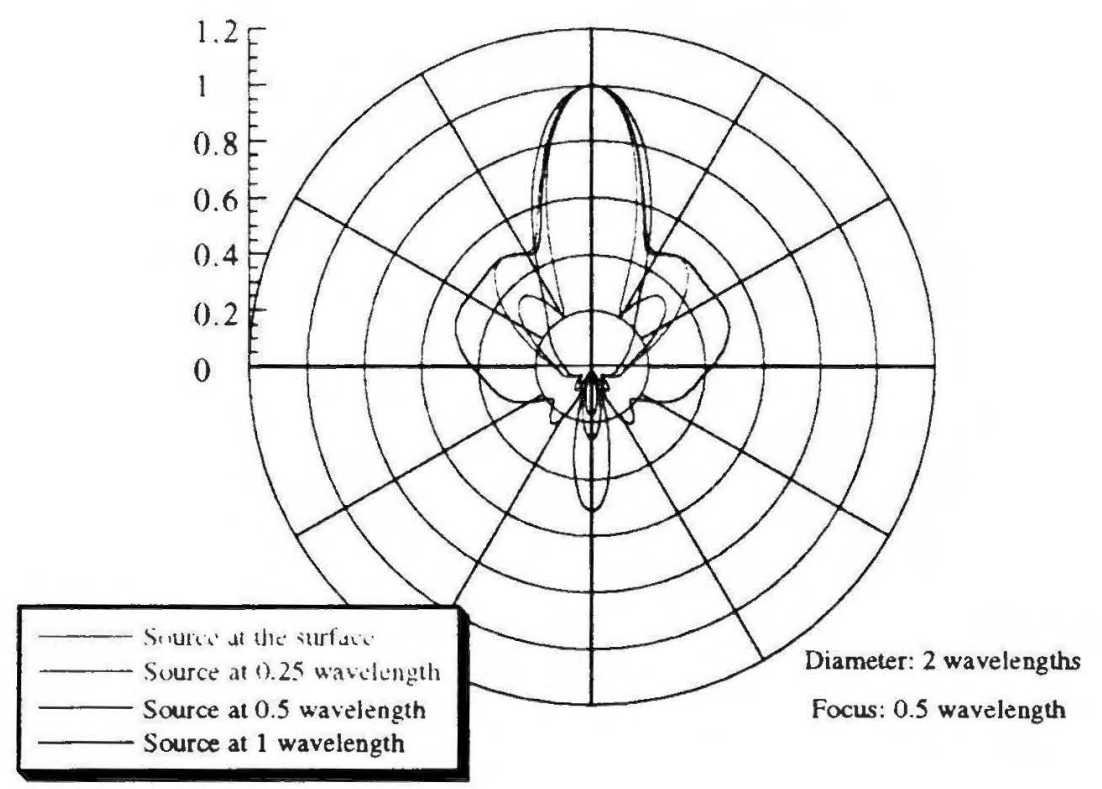

Figure 3.12 The BOR simulations of the parabolic reflector 2 


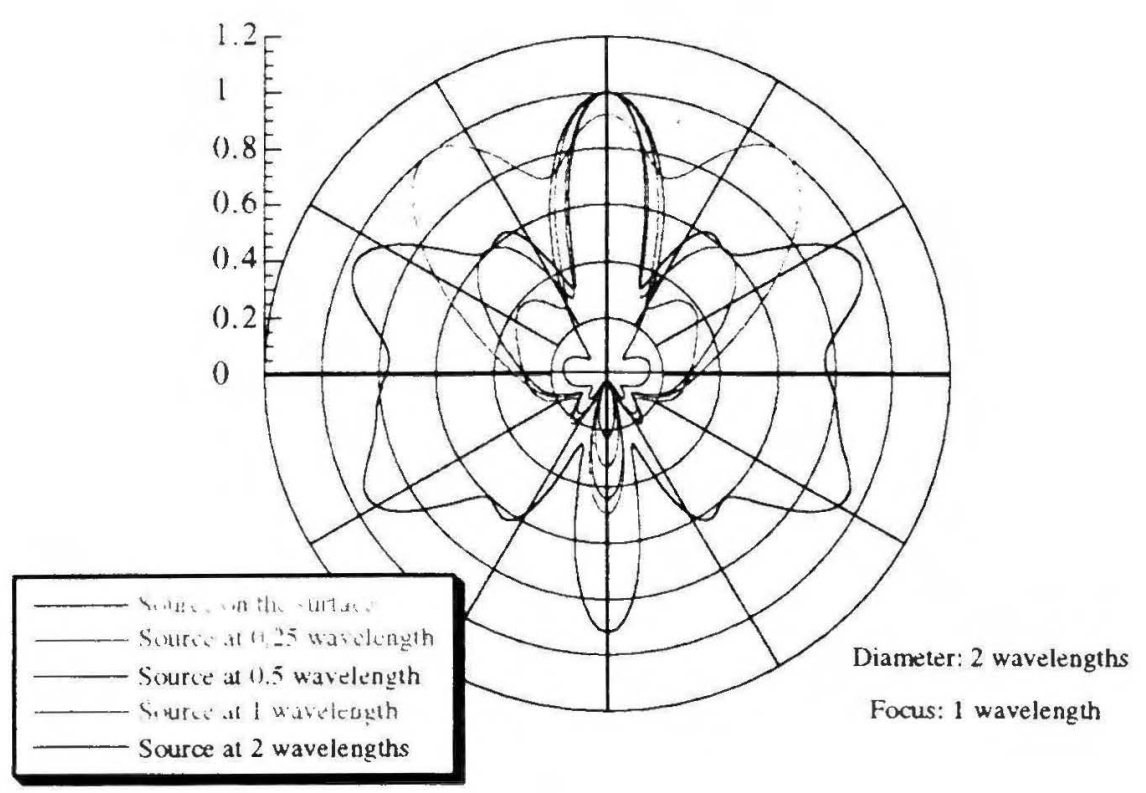

Figure 3.13 The BOR simulations of the parabolic reflector 3

Total pressure field amplitude in the azimuthal plane

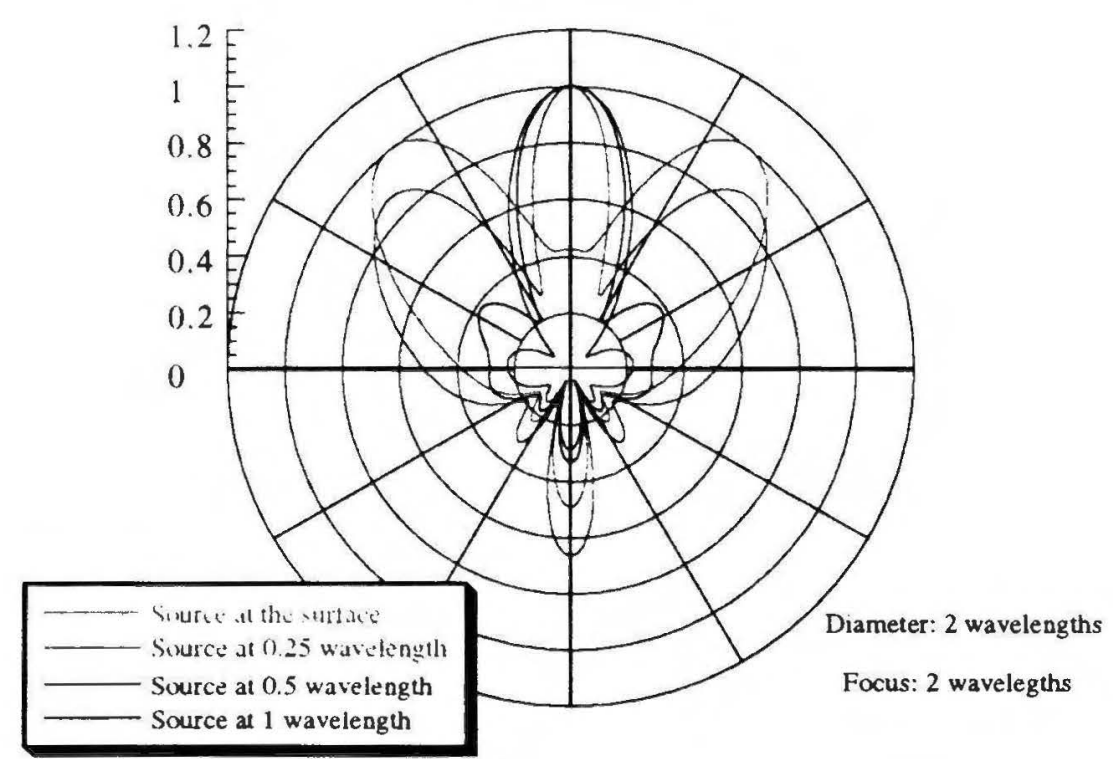

Figure 3.14 The BOR simulations of the parabolic reflector 4 
Total pressure field amplitude in the azimuthal plane

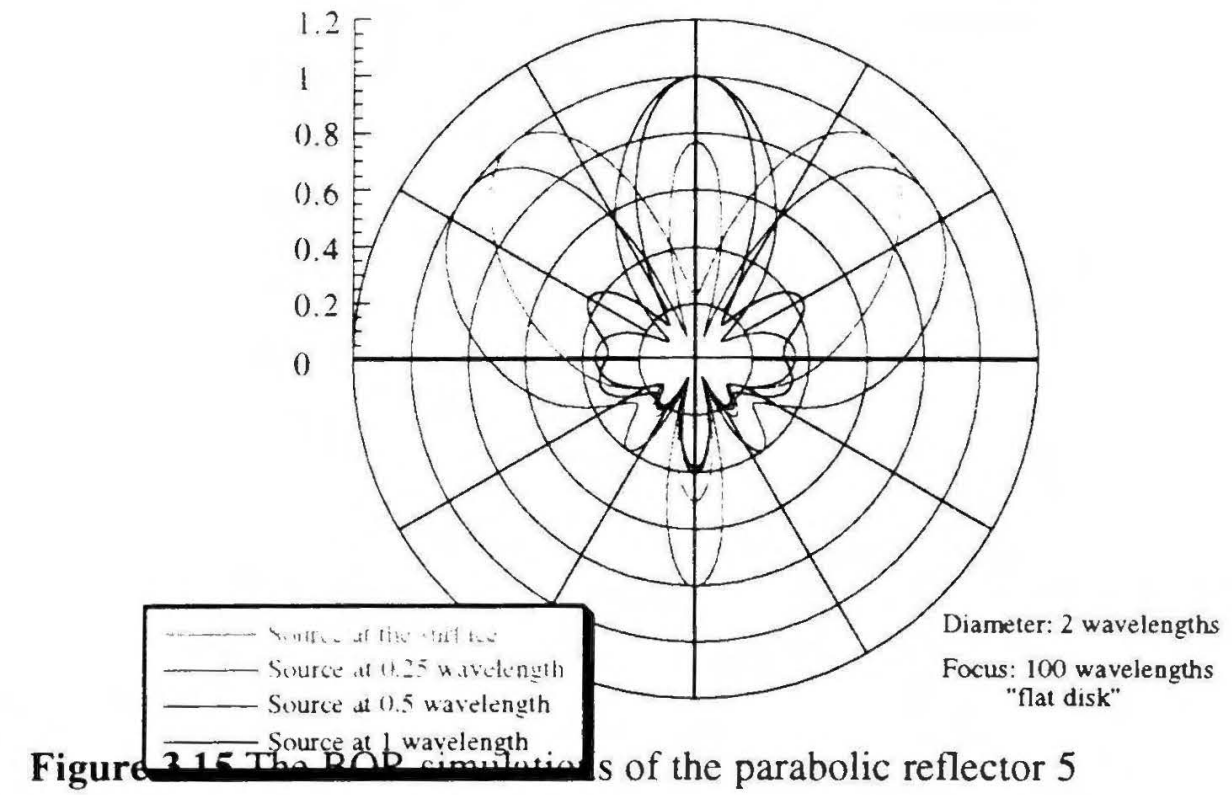

Total pressure field amplitude in the azimuthal plane

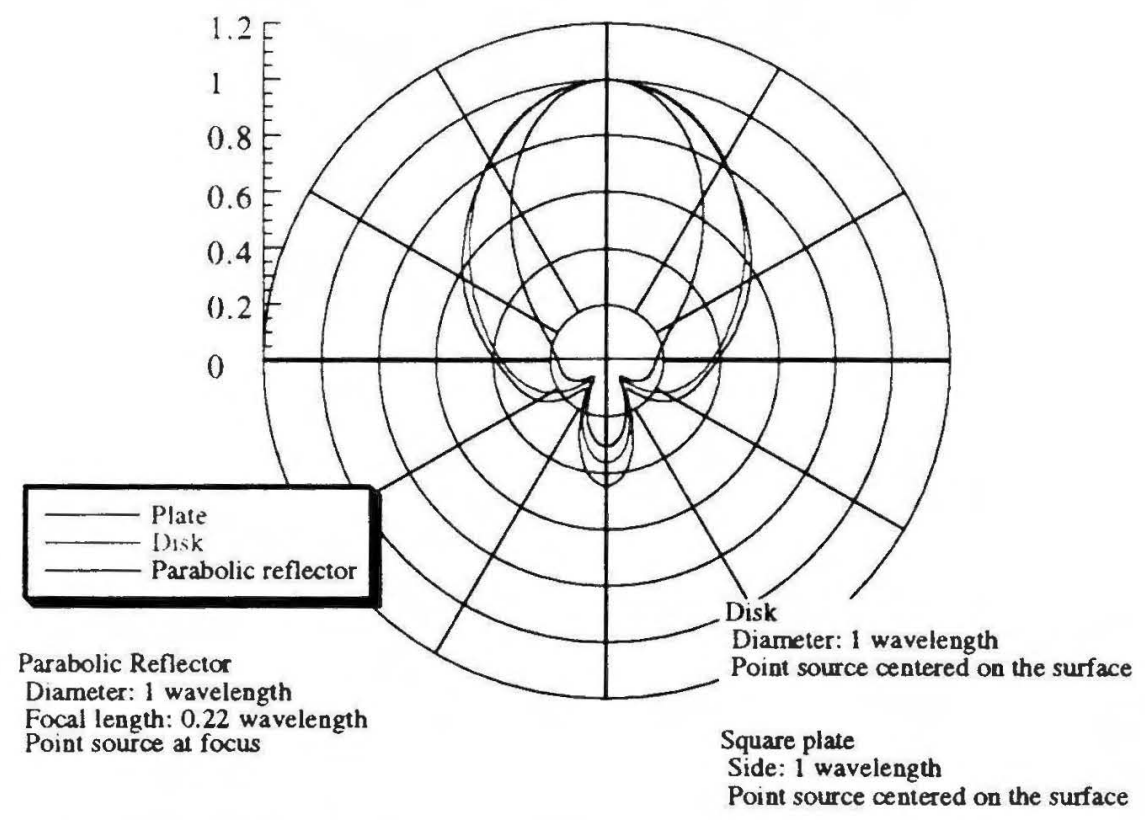

Figure 3.16 The BOR simulations of the parabolic reflector, disk, and baffle 


\subsection{Scattering From Thin Disks}

Although the parabolic reflector simulations due to a point source could not be compared to known results, the simulations can still be checked for the validity. When the focal length of the parabolic reflector becomes large compared to the wavelength, the shape of the reflector resembles a thin disk. Diffraction of a thin disk has been rigorously analyzed previously by many scholars $[7,9$, and 10$]$. Leitner presented the diffraction of sound by a circular disk due to a plane wave incidence [7], and the exact theoretical values based on the wave functions of the oblate spheroid were plotted in his paper. Also Wiener's measurements supported Leitner's work [10].

In order to compare with the Leitner's results, the same test cases were run using BOR code (see Figure 3.17). Figures 3.18, 3.19, 3.20, 3.21, and 3.22 show the BOR simulation results. The BOR simulation results agree with the Leitner's exact theoretical calculations, and they match closer to the experimental data than the Leitner's results [9].

There is no study on the diffraction patterns due to a point source incidence although an exact solution exists. The solution is expanded in terms of oblate spheroidal wave functions which are not simple to calculate. Since the oblate spheroidal wave functions are not available in any mathematical software package, a computer code is written to calculate the functions which are discussed in Chapter 4.

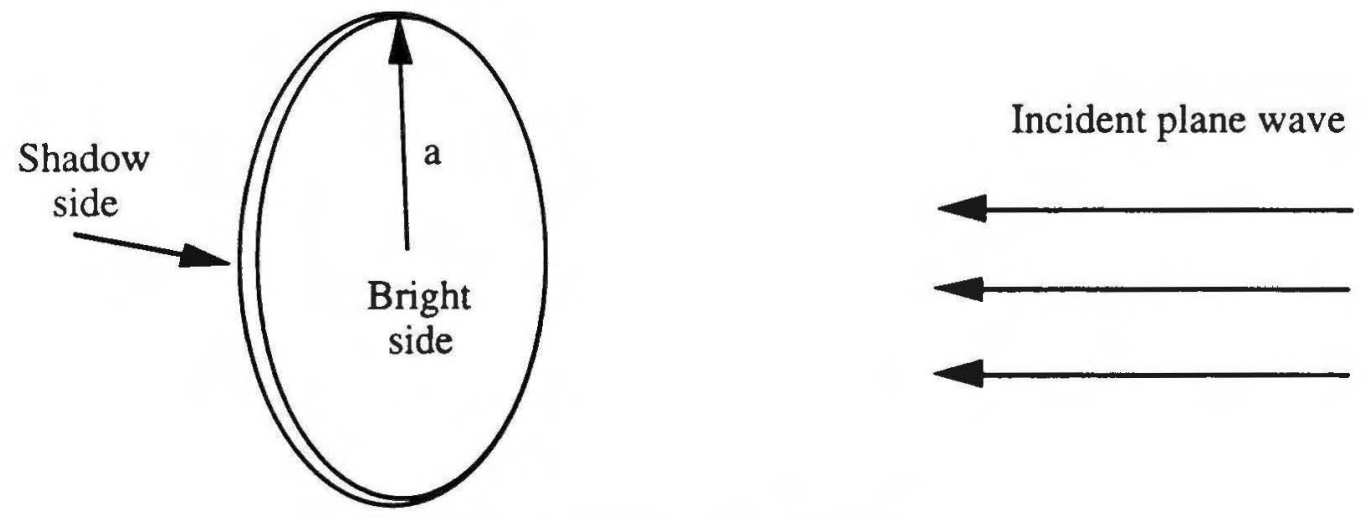

Figure 3.17 The thin disk 
Total pressure field amplitude on the surface of the disk

$\mathrm{ka}=1$

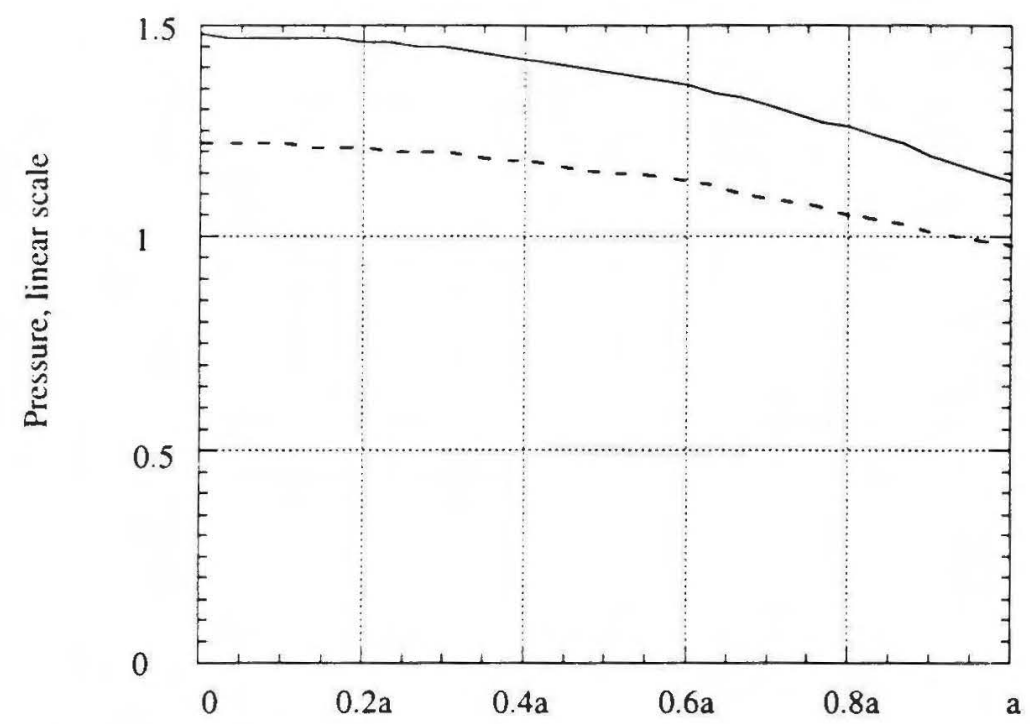

Bright side

Distance from the center of the disk

Figure 3.18 The BOR simulation of the disk 1

Total pressure field amplitude on the surface of the disk

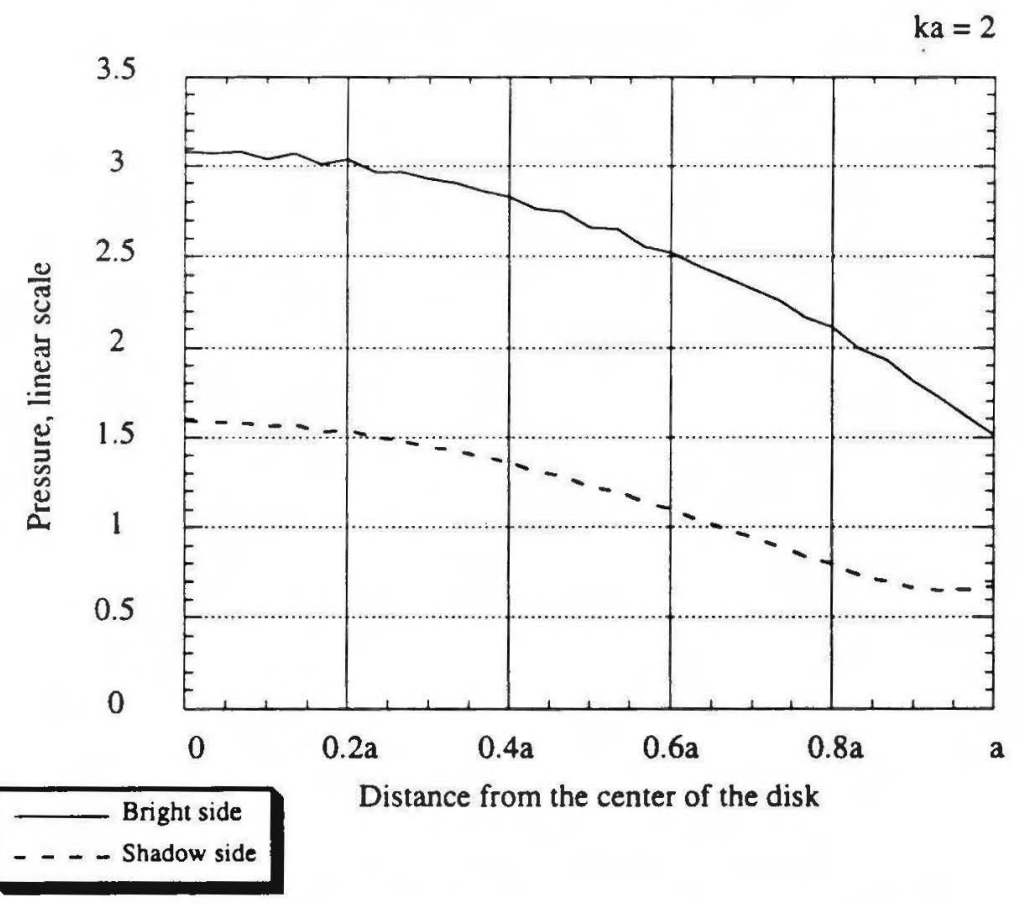

Figure 3.19 The BOR simulation of the disk 2 
Total pressure field amplitude on the surface of the disk

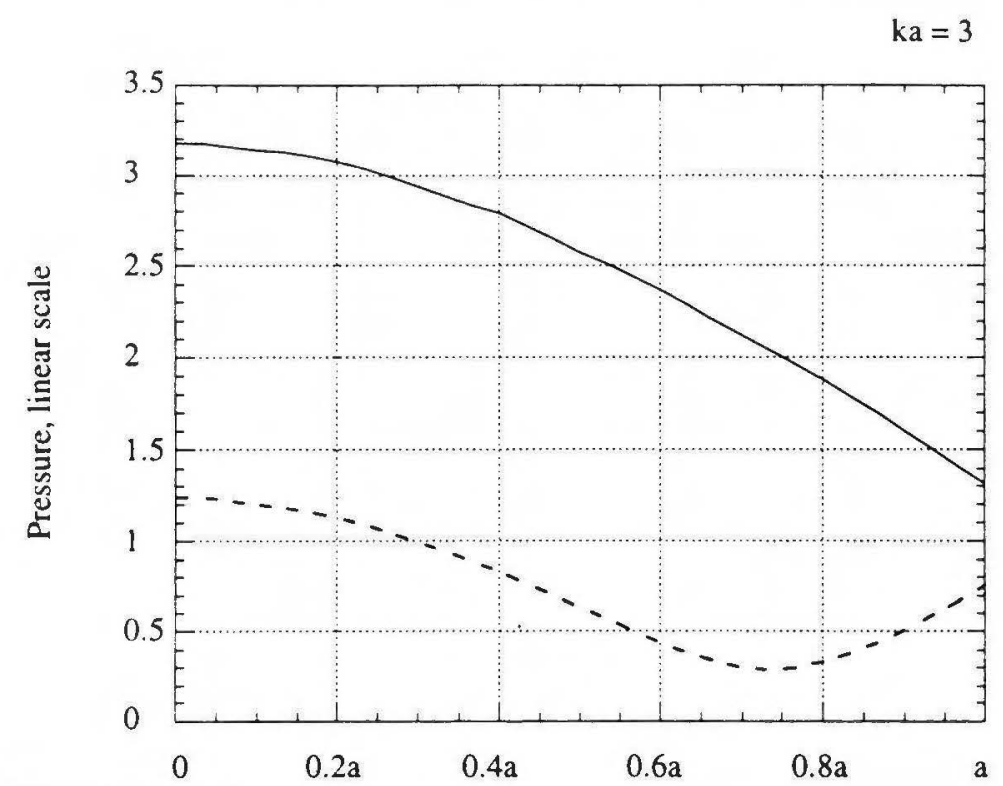

Bright side

Shadow side

Distance from the center of the disk

Figure 3.20 The BOR simulation of the disk 3

Total pressure field amplitude on the surface of the disk

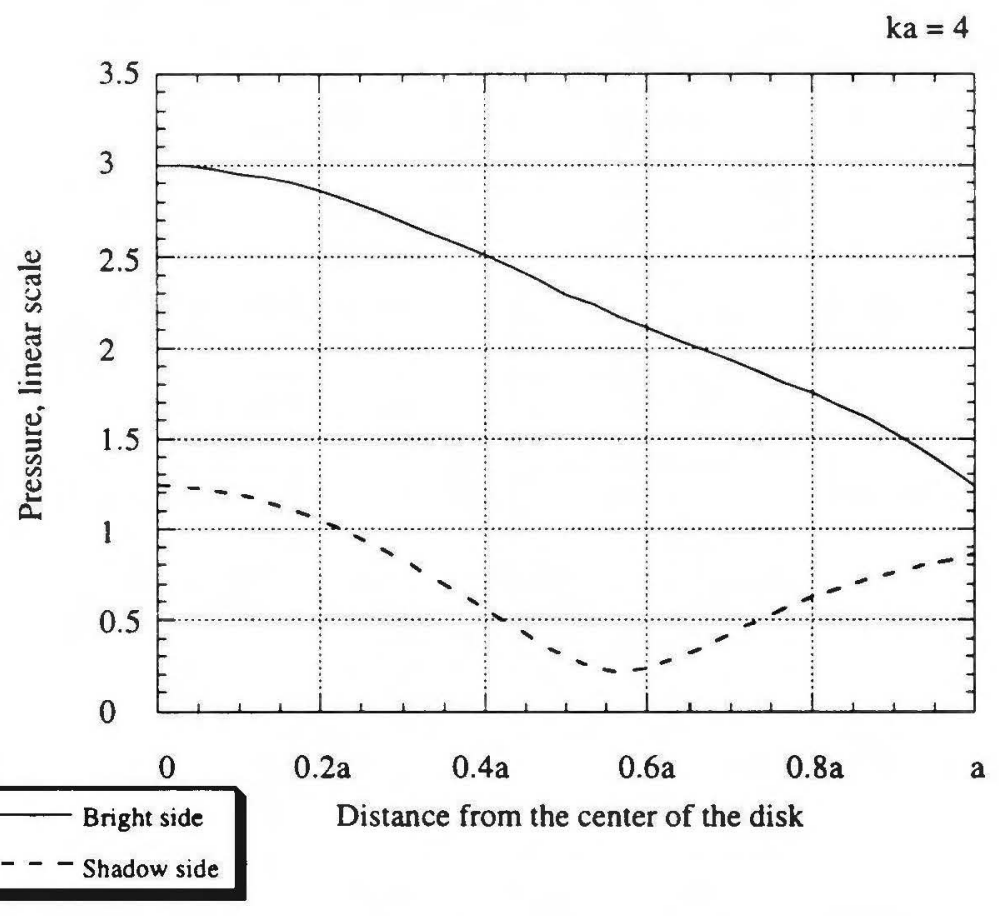

Figure 3.21 The BOR simulation of the disk 4 
Total pressure amplitude on the surface of the disk

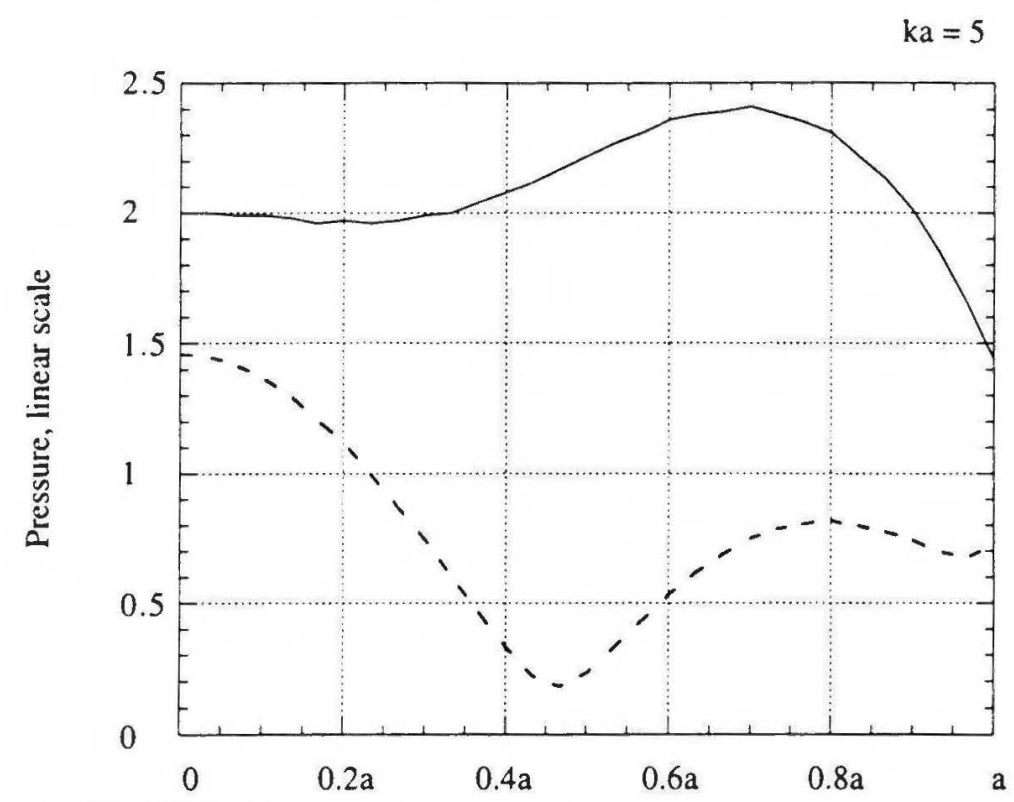

- Bright side

Distance from the center of the disk

Figure 3.22 The BOR simulation of the disk 5 


\section{CHAPTER 4 \\ SPHEROIDAL WAVE FUNCTIONS}

Chapter 3 describes BOR in detail and presents the BOR simulation results compared with the experimental, analytic and numerical solutions from $[5,6,7,8,9$, and 10]. However those results are valid only for an incident plane wave, and no solution is available for a point source excitation. In order to verify the BOR scattered patterns for a point source excitation, an analytic solution for an axisymmetric geometry must be sought. Studying the oblate spheroidal geometry is a good way to verify the BOR codes. A "fat" spheroid represents a sphere, and a thin spheroid becomes a disk.

\subsection{The Oblate Spheroidal Geometry}

The oblate spheroidal coordinates $(\xi, \eta, \phi)$ shown in Figure 4.1 are related to the rectangular Cartesian coordinates $(\mathrm{x}, \mathrm{y}, \mathrm{z})$ by the transformation

$$
\begin{aligned}
& x=\frac{1}{2} d \sqrt{\left(\xi^{2}+1\right)\left(1-\eta^{2}\right)} \cos \phi \\
& y=\frac{1}{2} d \sqrt{\left(\xi^{2}+1\right)\left(1-\eta^{2}\right)} \sin \phi \\
& z=\frac{1}{2} d \xi \eta
\end{aligned}
$$

where $0 \leq \xi<\infty,-1 \leq \eta \leq 1$, and $0 \leq \phi<2 \pi$. The $z$-axis is the axis of symmetry, and the interfocal distance, minor axis, and major axis are $d, d \xi$ and $d \sqrt{\xi^{2}+1}$, respectively [6]. Because of the axisymmetry there is no $\phi$ variation, so $\phi$ is assumed to be zero in the rest of this chapter.

The exact solution for an acoustically hard oblate spheroid due to a point source excitation on z-axis $\left(\eta_{0}=0\right)$ is computed by Bowman et al. [7] as 


$$
\frac{1}{\widetilde{N}_{0 n}}\left[R_{0 n}^{(1)}\left(-i c, i \xi_{<}\right)-\frac{R_{0 n}^{(1)^{\prime}}\left(-i c, i \xi_{1}\right)}{R_{0 n}^{(3)^{\prime}}\left(-i c, i \xi_{1}\right)} R_{0 n}^{(3)}\left(-i c, i \xi_{<}\right)\right] R_{0 n}^{(3)}\left(-i c, i \xi_{>}\right) S_{0 n}(-i c, \eta)
$$

The above notations are consistent with the Flammer's notations [11]. The coordinate of the oblate spheroid surface, the source point, and the field point are denoted as $\left(\xi_{1}, \eta_{1}, 0\right)$, $\left(\xi_{0}, 0,0\right)$, and $(\xi, \eta, \phi)$ respectively. Also $\xi_{<}$and $\xi_{>}$take the minimum and maximum values between the source point and the field point coordinates respectively.

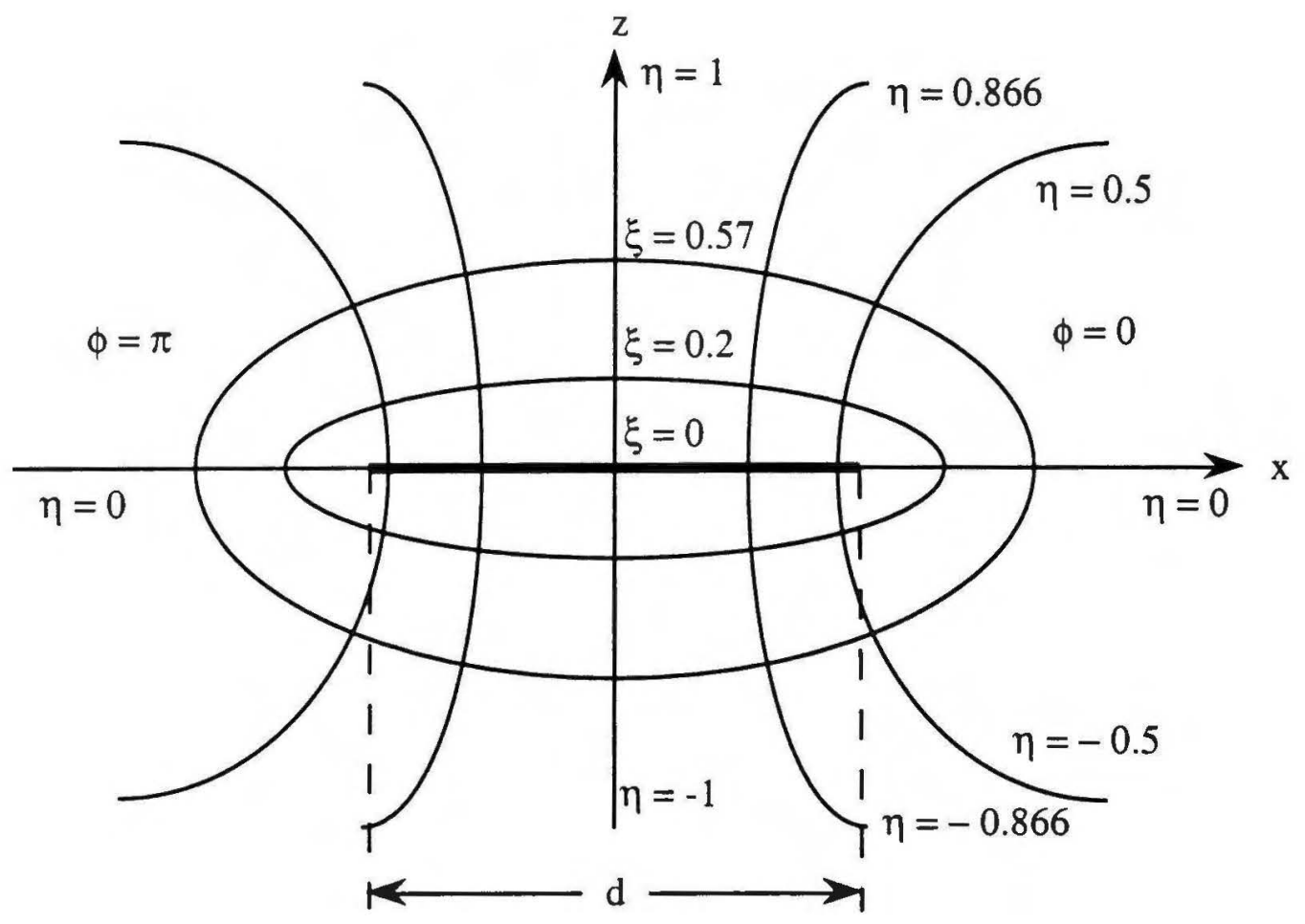

Figure 4.1 The oblate spheroidal coordinate system

The oblate spheroidal coordinate system is one of the coordinate systems in which the scalar wave equation

$$
\left(\nabla^{2}+k^{2}\right) \psi=0
$$

is separable [11]. To express this equation in spheroidal coordinates, the following relation for metrical coefficients, $h_{\xi}, h_{\eta}$, and $h_{\phi}$ are defined by 


$$
d x^{2}+d y^{2}+d z^{2}=h_{\xi}^{2} d \xi^{2}+h_{\eta}^{2} d \eta^{2}+h_{\phi}^{2} d \phi^{2}
$$

where the scale factors are defined in [12] as,

$$
\begin{gathered}
h_{\xi}=\frac{d}{2}\left(\frac{\xi^{2}+\eta^{2}}{\xi^{2}+1}\right)^{1 / 2}, h_{\eta}=\frac{d}{2}\left(\frac{\xi^{2}+\eta^{2}}{1-\eta^{2}}\right)^{1 / 2} \\
h_{\phi}=\frac{d}{2}\left[\left(1-\eta^{2}\right)\left(\xi^{2}+1\right)\right]^{1 / 2}
\end{gathered}
$$

With the use of the expression for the Laplacian operator in orthogonal curvilinear coordinates, the following differential equation is obtained [12].

$$
\left[\frac{\partial}{\partial \eta}\left(1-\eta^{2}\right) \frac{\partial}{\partial \eta}+\frac{\partial}{\partial \xi}\left(\xi^{2}+1\right) \frac{\partial}{\partial \xi}+\frac{\xi^{2}+\eta^{2}}{\left(\xi^{2}+1\right)\left(1-\eta^{2}\right)} \frac{\partial^{2}}{\partial \phi^{2}}+c^{2}\left(\xi^{2}+\eta^{2}\right)\right] \psi=0
$$

where $\mathrm{c}=\frac{1}{2} \mathrm{kd}$. By the usual procedure of the separation of variables, the solution of (4.6) is found as

$$
\psi_{\mathrm{mn}}=\mathrm{S}_{\mathrm{mn}}(-\mathrm{ic}, \eta) \mathrm{R}_{\mathrm{mn}}(-\mathrm{ic}, \mathrm{i} \xi) \exp (\mathrm{im} \phi)
$$

The angle and radial functions, $S_{m n}(-i c, \eta)$ and $R_{m n}(-i c, i \xi)$, satisfy the ordinary differential equations [11]

$$
\begin{aligned}
& \frac{\partial}{\partial \xi}\left[\left(\xi^{2}+1\right) \frac{\partial}{\partial \xi} S_{m n}(-i c, i \xi)\right]-\left[\lambda_{m n}(-i c)-c^{2} \xi^{2}-\frac{m^{2}}{\xi^{2}+1}\right] R_{m n}(-i c, i \xi)=0 \\
& \frac{\partial}{\partial \eta}\left[\left(1-\eta^{2}\right) \frac{\partial}{\partial \eta} S_{m n}(-i c, \eta)\right]+\left[\lambda_{m n}(-i c)+c^{2} \eta^{2}-\frac{m^{2}}{1-\eta^{2}}\right] S_{m n}(-i c, \eta)=0
\end{aligned}
$$

The angle and radial functions are the key functions in computing (4.2). All of the variables in (4.2) are evaluated at $m=0$, and the rest of this chapter assumes $m=0$. Flammer describes the derivations of the angle and radial functions in detail [11], and the following two sections briefly explain his derivations since understanding the derivations are crucial in developing a computer code. 


\subsection{The Angle Functions}

In the differential equation (4.9), $S_{0 n}$ is obtained from the expansions in the associated Legendre functions of the first kind [11]:

$$
S_{0 n}(-i c, \eta)=\sum_{r=0,1}^{\infty} d_{r}^{0 n}(-i c) P_{r}(\eta)
$$

The prime over the summation sign indicates that the summation is over only even values of

$r$ when $n$ is even, and over only odd values of $r$ when $n$ is odd. Substituting (4.10) in (4.9) and the use of the associated Legendre differential equation and the recursion formulas for the associated Legendre functions yield the following recursion formula for the coefficients $\mathrm{d}_{r}^{0 \mathrm{n}}[11]$ :

$$
\begin{gathered}
\frac{(r+2)(r+1) c^{2}}{(2 r+3)(2 r+5)} d_{r+2}^{0 n} \\
-\left[r(r+1)-\lambda_{0 n}-\frac{r(r+1)-1}{(2 r-1)(2 r+3)} c^{2}\right] d_{r}^{0 n}+\frac{r(r-1) c^{2}}{(2 r-2)(2 r-1)} d_{r-2}^{0 n}=0 \quad(r \geq 0)
\end{gathered}
$$

Finding the solution to the above recursion formula is the key step in obtaining the angle functions since the associated Legendre functions are relatively well-known functions. A FORTRAN subroutine from Numerical Recipes was used for the Legendre functions (see Appendix C).

For convenience, let

$$
\begin{gathered}
\gamma_{r}=r(r+1)-\frac{1}{2} c^{2}\left[1+\frac{1}{(2 r-1)(2 r+3)}\right], \quad(r \geq 0) \\
\beta_{r}=\frac{r^{2}(r-1)^{2} c^{4}}{(2 r-1)^{2}(2 r-3)(2 r+1)}, \quad(r \geq 2) \\
N_{r}=-\frac{r(r-1) c^{2}}{(2 r-1)(2 r+1)} \frac{d_{r}^{0 n}}{d_{r-2}^{0 n}}, \quad(r \geq 2)
\end{gathered}
$$

Substituting above notations in (4.11) yields, 


$$
N_{r}=\frac{\beta_{r}}{\gamma_{r}-\lambda_{0 n}-N_{r+2}},(r \geq 2)
$$

and reciprocally

$$
N_{r+2}=\lambda_{0 n}-\gamma_{T}-\frac{\beta_{r}}{N_{r}}, \quad(r \geq 2)
$$

with $\mathrm{N}_{2}=\lambda_{0 \mathrm{n}}-\gamma_{0}, \mathrm{~N}_{2}=\lambda_{0 \mathrm{n}}-\gamma_{1}$.

The recursion formula (4.11) must be convergent, and this condition leads to the iterative computations of $\mathrm{N}_{\mathrm{r}}$ from (4.14) for large values of $\mathrm{r}$. Conversely from the initial values $\mathrm{N}_{2}$ and $\mathrm{N}_{3}, \mathrm{~N}_{\mathrm{r}}$ is calculated from (4.15) for small values of $\mathrm{r}$. When $\mathrm{c}$ is small, the dominant coefficient for given $n$ is $d_{n}^{0 n}$ [11]. Thus it is convenient to obtain the ratios of the coefficients $d_{r}^{0 n} / d_{n}^{0 n}$. It is observed that the series of the coefficient ratios obtained from (4.15) is accurate only for large $r$, and the series from (4.14) is accurate only for small $r$. Therefore for $r$ less than $n$, the coefficient ratios from (4.14) are taken, and for $r$ greater than $\mathrm{n}$, the coefficient ratios from (4.15) are taken.

The calculation of the coefficients are based on the accurate value of $\lambda_{0 n}$. The eigenvalue for a small $c^{2}$ is obtained from a series in powers of $c^{2}[11]$.

$$
\lambda_{0 \mathrm{n}}(-\mathrm{ic})=\sum_{\mathrm{k}=0}^{\infty}(-1)^{\mathrm{k}} 1_{2 \mathrm{k}}^{0 \mathrm{n}} \mathrm{c}^{2 \mathrm{k}}
$$

where

$$
\begin{gathered}
\mathrm{I}_{0}^{0 \mathrm{n}}=\mathrm{n}(\mathrm{n}+1) \\
1_{2}^{0 \mathrm{n}}=\frac{1}{2}\left[1+\frac{1}{(2 n-1)(2 \mathrm{n}+3)}\right] \\
1_{4}^{0 \mathrm{n}}=\frac{-(\mathrm{n}+1)^{2}(\mathrm{n}+2)^{2}}{2(2 \mathrm{n}-1)(2 \mathrm{n}+2)(2 \mathrm{n}+3)^{3}(2 \mathrm{n}+5)}+\frac{\mathrm{n}^{2}(\mathrm{n}-1)^{2}}{2(2 \mathrm{n}-3)(2 \mathrm{n}-1)^{3}(2 \mathrm{n}+1)}
\end{gathered}
$$

$I_{6}^{0}=\frac{n^{2}(n-1)^{2}}{(2 n-5)(2 n-3)(2 n-1)^{5}(2 n+1)(2 n+3)}+\frac{(n+1)^{2}(n+2)^{2}}{(2 n-1)(2 n+2)(2 n+3)^{5}(2 n+5)(2 n+7)}$

$$
\begin{aligned}
& 1_{8}^{0 n}=2 A+\frac{1}{16} B+\frac{1}{8} C+\frac{1}{2} D \\
& A=\frac{n^{2}(n-1)^{2}}{(2 n-5)^{2}(2 n-3)(2 n-1)^{7}(2 n+1)(2 n+3)^{2}}-\frac{(n+1)^{2}(n+2)^{2}}{(2 n-1)^{2}(2 n+1)(2 n+3)^{7}(2 n+5)(2 n+7)^{2}}
\end{aligned}
$$




$$
\begin{gathered}
B=\frac{n^{2}(2 n-1)^{2}(2 n-2)^{2}(2 n-3)^{2}}{(2 n-7)(2 n-5)^{2}(2 n-3)^{3}(2 n-1)^{4}(2 n+1)}-\frac{(n+1)^{2}(n+2)^{2}(n+3)^{2}(n+4)^{2}}{(2 n+1)(2 n+3)^{4}(2 n+5)^{3}(2 n+7)^{2}(2 n+9)} \\
C=\frac{(n+1)^{4}(n+2)^{4}}{(2 n+1)^{2}(2 n+3)^{7}(2 n+5)^{2}}-\frac{n^{4}(n-1)^{4}}{(2 n-3)^{2}(2 n-1)^{7}(2 n+1)^{2}} \\
D=\frac{(n-1)^{2} n^{2}(n+1)^{2}(n+2)^{2}}{(2 n-3)(2 n-1)^{4}(2 n+1)^{2}(2 n+3)^{4}(2 n+5)}
\end{gathered}
$$

In the numerical computation, truncating the summation up to $\mathrm{k}=4$ yields sufficiently accurate eigenvalues for small $\mathrm{c}^{2}$. For a large argument, an asymptotic expansion is used [11].

$$
\lambda_{0 n}(-i c)=-c^{2}+2 c(2 v+1)-2 v(v+1)-1+\Lambda_{0 n}
$$

where $v=\frac{\mathrm{n}}{2}$ for $\mathrm{n}$ even and $v=\frac{\mathrm{n}-1}{2}$ for $\mathrm{n}$ odd. $\Lambda_{0 \mathrm{n}}$ is defined as

$$
\begin{gathered}
\Lambda_{0 n}=\sum_{k=1}^{\infty} \beta_{k}^{0 n} c^{-k} \\
\beta_{1}^{0 n}=-2^{-3} q\left(q^{2}+1\right), \quad \beta_{2}^{0 n}=-2^{-6}\left[5 q^{4}+10 q^{2}+1\right] \\
\beta_{3}^{0 n}=-2^{-9} q\left[33 q^{4}+114 q^{2}+37\right], \quad \beta_{4}^{0 n}=-2^{-10}\left[63 q^{6}+340 q^{4}+239 q^{2}+14\right]
\end{gathered}
$$

where $\mathrm{q}=\mathrm{n}+1$ for $\mathrm{n}$ even and $\mathrm{q}=\mathrm{n}$ for $\mathrm{n}$ odd .

The power series and asymptotic expansion methods are not sufficient for intermediate values of $\mathrm{c}^{2}$, and the eigenvalue should be refined by Boukamp's method of approximation. The eigenvalue is obtained from a transcendental equation $U$ which is originated from equations (4.15) and (4.16) [11]

$$
\mathrm{U}\left(\lambda_{O_{n}}\right)=\mathrm{U}_{1}\left(\lambda_{0_{n}}\right)+\mathrm{U}_{2}\left(\lambda_{O_{n}}\right)=0
$$

where 


$$
\mathrm{U}_{1}\left(\lambda_{0 \mathrm{n}}\right)=-\gamma_{\mathrm{n}}+\lambda_{0 \mathrm{n}}-\frac{\beta_{\mathrm{n}}}{\gamma_{\mathrm{n}-2}-\lambda_{0 \mathrm{n}}-\mathrm{N}_{\mathrm{n}-2}} \quad \text { and } \quad \mathrm{U}_{1}\left(\lambda_{0 \mathrm{n}}\right)=-\frac{\beta_{\mathrm{n}+2}}{\gamma_{\mathrm{n}+2}-\lambda_{0 \mathrm{n}}-N_{n+4}}
$$

Note that $N_{n-2}$ is evaluated by the equation (4.16) and $N_{n+4}$ is evaluated by (4.15). Let $\lambda_{0 n}^{(1)}$ be the approximate eigenvalue calculated from either of the above two methods, and $\delta \lambda_{0 \mathrm{n}}$ be the difference between the actual eigenvalue and the approximate value.

$$
0=U\left(\lambda_{0 n}\right)=U\left(\lambda_{0 n}^{(1)}+\delta \lambda_{0 n}\right) \approx U\left(\lambda_{0 n}^{(1)}\right)+\delta U
$$

by finding the first variation of $\mathrm{U}$ due to the variation $\delta \lambda_{0 \mathrm{n}}$. If the variation $\delta \lambda_{0 \mathrm{n}}$ is made in the eigenvalue, the variation on (4.16) is

$$
\delta N_{r+2}=\delta \lambda_{0 n}+\frac{\beta_{r}}{\left(N_{r}\right)^{2}} \delta N_{r}
$$

Likewise, the variation on (4.15) is

$$
\delta N_{r}=\frac{\left(N_{r}\right)^{2}}{\beta_{r}}\left[\delta N_{r+2}-\delta \lambda_{0 n}\right]
$$

By iteration of (4.23) and (4.24) the variations of $U$ are obtained

$$
\begin{gathered}
\delta U_{1}=\delta \lambda_{0 n}\left[1+\frac{\beta_{n}}{\left(N_{n}\right)^{2}}+\frac{\beta_{n} \beta_{n-2}}{\left(N_{n}\right)^{2}\left(N_{n-2}\right)^{2}}+\frac{\beta_{n} \beta_{n-2} \beta_{n-4}}{\left(N_{n}\right)^{2}\left(N_{n-2}\right)^{2}\left(N_{n-4}\right)^{2}}+\cdots\right] \\
\delta U_{2}=\delta \lambda_{0 n}\left[\frac{\left(N_{n}\right)^{2}}{\beta_{n}}+\frac{\left(N_{n}\right)^{2}\left(N_{n+2}\right)^{2}}{\beta_{n} \beta_{n+2}}+\frac{\left(N_{n}\right)^{2}\left(N_{n+2}\right)^{2}\left(N_{n+4}\right)^{2}}{\beta_{n} \beta_{n+2} \beta_{n+4}}+\cdots\right]
\end{gathered}
$$

Substituting (4.25) and (4.26) in (4.22), $\delta \lambda_{0 \mathrm{n}}$ is found to be

$$
\delta \lambda_{0 n}=\frac{-U_{1}\left(\lambda_{0 n}^{(1)}\right)-U_{2}\left(\lambda_{0 n}^{(1)}\right)}{\left[1+\frac{\beta_{n}}{\left(N_{n}\right)^{2}}+\frac{\beta_{n} \beta_{n-2}}{\left(N_{n}\right)^{2}\left(N_{n-2}\right)^{2}}+\cdots\right]+\left[\frac{\left(N_{n+2}\right)^{2}}{\beta_{n+2}}+\frac{\left(N_{n+2}\right)^{2}\left(N_{n+4}\right)^{2}}{\beta_{n+2} \beta_{n+4}}+\cdots\right]}
$$

The new eigenvalues obtained by the above method yield exactly same eigenvalues listed in [12]. Thus the Boukamp's method leads to remarkably accurate eigenvalues, and this 
accuracy is the key in calculating the coefficients.

Once the coefficient ratios are determined accurately, the actual values of the coefficients can be obtained in terms of an arbitrary coefficient value. Flammer uses a normalization scheme [12] such that each spheroidal angle function reduces exactly to the corresponding associated Legendre function when $\mathrm{c}$ becomes zero. This normalization follows Chu and Stratton's normalization scheme [12] except that the normalization is carried out at $\eta=0$. Thus using the normalization relations below, the coefficients are completely determined.

$$
\begin{gathered}
\sum_{r=0}^{\infty} \frac{(-1)^{2} r !}{2^{r}\left(\frac{r}{2}\right)^{2} !} d_{r}^{0 n}=\frac{(-1)^{n / 2} n !}{2^{n}\left(\frac{n}{2}\right)^{2 !}} \quad \text { for } n \text { even } \\
\sum_{r=1}^{\infty} \frac{(-1)^{\frac{r-1}{2}}(r+1) !}{2^{r}\left(\frac{r-1}{2}\right) !\left(\frac{r+1}{2}\right) !} d_{r}^{0 n}=\frac{(-1)^{\frac{n-1}{2}}(n+1) !}{2^{n}\left(\frac{n-1}{2}\right) !\left(\frac{n+1}{2}\right) !} \quad \text { for } n \text { odd }
\end{gathered}
$$

Once the expansion coefficients are determined, $\widetilde{\mathrm{N}}_{n_{n}}$ from (4.2) is easily found as below [11].

$$
\widetilde{N}_{0 n}=2 \sum_{r=0,1}^{\infty} \frac{r !\left(d_{r}^{0 n}\right)^{2}}{(2 r+1) r !}
$$

\subsection{The Radial Functions}

The radial functions satisfy the differential equation (4.9). The eigenvalue in (4.9) is identical as in (4.8) of the angle function. The radial functions are found in terms of the spherical Bessel, Neumann, and Hankel functions [8].

$$
R_{0 n}^{(p)}(-i c, i \xi)=\left[\sum_{r=0,1}^{\infty} d_{r}^{0 n}\right]^{-1} \sum_{r=0,1}^{\infty} i^{r-n} d_{r}^{0 n} Z_{r}^{(p)}(c \xi)
$$

where

$$
\begin{array}{ll}
Z_{T}^{(p)}(z)=\sqrt{\frac{\pi}{2 z}} J_{r+\frac{1}{2}}(z) & \text { for } p=1 \\
Z_{T}^{(p)}(z)=\sqrt{\frac{\pi}{2 z}} Y_{r+\frac{1}{2}}(z) & \text { for } p=2
\end{array}
$$


$\mathrm{J}$ and $\mathrm{Y}$ are Bessel and Neumann functions, respectively. Similar to Hankel functions, the radial functions are related likewise [12]

$$
\begin{aligned}
& \mathrm{R}_{0 \mathrm{n}}^{(3)}(-\mathrm{ic}, \mathrm{i} \xi)=\mathrm{R}_{0 \mathrm{n}}^{(1)}(-\mathrm{ic}, \mathrm{i} \xi)+\mathrm{i} \mathrm{R}_{0 \mathrm{n}}^{(2)}(-\mathrm{ic}, \mathrm{i} \xi) \\
& \mathrm{R}_{0 \mathrm{n}}^{(4)}(-\mathrm{ic}, \mathrm{i} \xi)=\mathrm{R}_{0 \mathrm{n}}^{(1)}(-\mathrm{ic}, \mathrm{i} \xi)-i \mathrm{R}_{0 \mathrm{n}}^{(2)}(-\mathrm{ic}, \mathrm{i} \xi)
\end{aligned}
$$

(4.33) makes defining the radial functions easier. The derivative of $R_{0 n}^{(3)}(-i c, i \xi)$ is easily obtained by taking the first derivatives of $R_{0 n}^{(1)}(-i c, i \xi)$ and $R_{0 n}^{(2)}(-i c, i \xi)$.

$$
R_{0 n}^{(p)^{\prime}}(-i c, i \xi)=\left[\sum_{r=0,1}^{\infty} d_{r}^{0 n}\right]^{-1} \sum_{r=0,1}^{\infty} i^{r-n} d_{r}^{0 n} Z_{r}^{(p)^{\prime}}(c \xi)
$$

However calculating the radial function of the second kind for small values of $\xi$ is not suitable since the Neumann function converges poorly for small arguments. For small values of $\xi$, a better numerical method should be used to calculate the radial function of the second kind.

The angle functions and the radial functions are related by joining factors $\kappa_{0 \mathrm{n}}^{(\mathrm{p})}[12]$,

$$
\mathrm{S}_{0 \mathrm{n}}^{(\mathrm{p})}(-\mathrm{ic}, \mathrm{i} \xi)=\kappa_{0 \mathrm{n}}^{(\mathrm{p})}(-\mathrm{ic}) \mathrm{R}_{0 \mathrm{n}}^{(\mathrm{p})}(-\mathrm{ic}, \mathrm{i} \xi) \quad \text { for } \mathrm{p}=1,2
$$

where

$$
\begin{gathered}
\kappa_{0 n}^{(1)}(-i c)=\frac{n ! \sum_{r=0}^{\prime} d_{r}^{0 n}}{2^{n} d_{0}^{0 n}\left(\frac{n}{2}\right)^{2 !}} \text { for } n \text { even } \\
\kappa_{0 n}^{(1)}(-i c)=-\frac{3(n+1) ! \sum_{r=1}^{\prime} d_{r}^{0 n}}{i c 2^{n} d_{1}^{0 n}\left(\frac{n-1}{2}\right) !\left(\frac{n+1}{2}\right) !} \text { for } n \text { odd } \\
\kappa_{0 n}^{(2)}(-i c)=\frac{i c 2^{n}\left(\frac{n}{2}\right)^{2 !} d_{0}^{0 n}}{n !} \sum_{r=0}^{\infty} d_{r}^{0 n} \text { for } n \text { even } \\
\kappa_{0 n}^{(2)}(-i c)=\frac{c^{2} 2^{n}\left(\frac{n-1}{2}\right) !\left(\frac{n+1}{2}\right) ! d_{1}^{0 n}}{3(n+1) !} \sum_{r=1}^{\infty} d_{r}^{0 n} \text { for } n \text { odd }
\end{gathered}
$$


Based on the above relations, Flammer has derived power series expansions for the oblate radial functions as below [11].

$$
\mathrm{R}_{0 \mathrm{n}}^{(2)}(-\mathrm{ic}, \mathrm{i} \xi)=\mathrm{Q}_{0 \mathrm{n}}^{*}(-\mathrm{ic}) \mathrm{R}_{0 \mathrm{n}}^{(1)}(-\mathrm{ic}, \mathrm{i} \xi)\left[\tan ^{-1}(\xi)-\frac{\pi}{2}\right]+\mathrm{g}_{0 \mathrm{n}}(-\mathrm{ic}, \mathrm{i} \xi)
$$

with

$$
\begin{aligned}
& Q_{0 n}^{*}(-i c)=\frac{\left[\kappa_{0 n}^{(1)}(-i c)\right]^{2}}{c} \alpha_{0}^{0 n}(-i c) \frac{(-2 r) !}{r !\left[2^{-r}(-r) !\right]^{2}} \text { for } n \text { even } \\
& Q_{0 n}^{*}(-i c)=\frac{\left[\kappa_{0 n}^{(1)}(-i c)\right]^{2}}{c} \alpha_{0}^{0 n}(-i c) \frac{(1-2 r) !}{r !\left[2^{-r}(-r) !\right]^{2}} \text { for } n \text { odd }
\end{aligned}
$$

where $\alpha_{0}^{0 \mathrm{n}}(-\mathrm{ic})=\frac{1}{\left[\mathrm{c}_{0}^{0 \mathrm{n}}\right]^{2}}$. The coefficients $\mathrm{c}_{2 \mathrm{k}}^{0 \mathrm{n}}$ are

$$
\begin{aligned}
& c_{2 k}^{0 n}=\frac{1}{(k !)^{2}} \sum_{r=k}^{\infty}(-r)_{k}\left(r+\frac{1}{2}\right)_{k} d_{2 r}^{0 n} \text { for } n \text { even } \\
& c_{2 k}^{0 n}=\frac{1}{(k !)^{2}} \sum_{r=k}^{\infty}(-r)_{k}\left(r+\frac{3}{2}\right)_{k} d_{2 r+1}^{0 n} \text { for } n \text { odd }
\end{aligned}
$$

where $(\mathrm{r})_{\mathrm{k}} \equiv \mathrm{r}(\mathrm{r}+1)(\mathrm{r}+2) \cdots(\mathrm{r}+\mathrm{k}-1),(\mathrm{r})_{0} \equiv 1$.

The function $\mathrm{g}_{0 \mathrm{n}}$ in (4.37) satisfies the inhomogeneous radial equation [11]

$$
\left[\frac{d}{d \xi}\left(\xi^{2}+1\right) \frac{d}{d \xi}-\lambda_{0 n}(-i c)+c^{2} \xi^{2}\right] g_{0 n}(-i c, i \xi)=-2 Q_{0 n}^{*}(-i c) \frac{d}{d \xi} R_{0 n}^{(1)}(-i c, i \xi)
$$

It is convenient to expand $g_{0 \mathrm{n}}$ in the form

$$
\begin{aligned}
g_{0 n}(-i c, i \xi) & =\sum_{r=0}^{\infty} B_{2 r}^{0 n} \xi^{2 r+1} \text { for } n \text { even } \\
g_{0 n}(-i c, i \xi) & =\sum_{r=n}^{\infty} B_{2 r}^{0 n} \xi^{2 r} \text { for } n \text { odd }
\end{aligned}
$$

since the power series expansions are used for the values of $\xi$ which are close to zero [11]. After substituting (4.41) in (4.40) and rearranging, the following recursion formulas for $B_{2 r}^{0 n}$ are obtained [11]. 


$$
\begin{gathered}
(2 r+2)(2 r+3) B_{2 r+2}^{0 n}+\left[(2 r+1)(2 r+2)-\lambda_{0 n}(-i c)\right] B_{2 r}^{0 n}+c^{2} B_{2 r-2}^{0 n} \\
=-2 Q_{0 n}^{*}(-i c)\left[\kappa_{0 n}^{(1)}(-i c)\right]^{-1} \sum_{k=r+1}^{\infty} c_{2 k}^{0 n}(-i c) 2 k(k+1) \text { for } n \text { even, } \\
(2 r+1)(2 r+2) B_{2 r+2}^{0 n}+\left[2 r(2 r+1)-\lambda_{0 n}(-i c)\right] B_{2 r}^{0 n}+c^{2} B_{2 r-2}^{0 n} \\
=-2 Q_{0 n}^{*}(-i c)\left[i^{-1} \kappa_{0 n}^{(1)}(-i c)\right]\left[\sum_{k=r}^{\infty} c_{2 k}^{0 n}(-i c)(2 k+1)\left(\begin{array}{l}
k \\
r
\end{array}\right)-\sum_{k=r+1}^{\infty} c_{2 k}^{0 n}(-i c) 2 k(k-1)\right] \text { for } n \text { odd }
\end{gathered}
$$

The symbol $\left(\begin{array}{l}\mathrm{k} \\ \mathrm{r}\end{array}\right)$ denotes the binomial coefficient $\frac{\mathrm{k} !}{(\mathrm{k}-\mathrm{r}) ! \mathrm{r} !}$. The initial coefficients $B_{0}^{0 \mathrm{n}}$ are found in [11] as

$$
\begin{gathered}
B_{0}^{0 n}=\left[c R_{0 n}^{(1)}(-i c, i 0)\right]^{-1}-Q_{0 n}^{*}(-i c) R_{0 n}^{(1)}(-i c, i 0) \text { for } n \text { even } \\
B_{0}^{0 n}=\left[c R_{0 n}^{(1)^{\prime}}(-i c, i 0)\right]^{-1} \text { for } n \text { odd }
\end{gathered}
$$

where

$$
\begin{gathered}
R_{0 n}^{(1)}(-i c, i 0)=\frac{i^{n} d_{0}^{0 n}(-i c)}{\sum_{r=0}^{\infty} d_{r}^{0 n}(-i c)} \text { for } n \text { even, } R_{0 n}^{(1)}(-i c, i 0)=0 \text { for } n \text { odd } \\
R_{0 n}^{(1)}(-i c, i 0)=\frac{2 c i^{n-1} d_{1}^{0 n}(-i c)}{3 \sum_{r=0}^{\infty} d_{r}^{0 n}(-i c)} \text { for } n \text { odd, } \quad R_{0 n}^{(1)^{\prime}}(-i c, i 0)=0 \text { for } n \text { even }
\end{gathered}
$$

The remaining coefficients are completely determined from the recursion formulas in (4.42) and (4.43).

The derivative of $R_{0 n}^{(2)}$ is determined by simply taking a derivative of (4.37).

$$
\mathrm{R}_{0 \mathrm{n}}^{(2)^{\prime}}(-\mathrm{ic}, \mathrm{i} \xi)=\mathrm{Q}_{0 \mathrm{n}}^{*}(-\mathrm{ic}) \mathrm{R}_{0 \mathrm{n}}^{(1)^{\prime}}(-\mathrm{ic}, \mathrm{i} \xi)\left[\tan ^{-1}(\xi)-\frac{\pi}{2}\right]+\frac{\mathrm{Q}_{0 \mathrm{n}}^{*}(-\mathrm{ic}) \mathrm{R}_{0 \mathrm{n}}^{(1)}(-\mathrm{ic}, \mathrm{i} \xi)}{\xi^{2}+1}+\mathrm{g}_{0 \mathrm{n}}^{\prime}(-\mathrm{ic}, \mathrm{i} \xi)
$$

where $g^{\prime}{ }_{0}$ is 


$$
\begin{gathered}
g_{\text {on }}^{\prime}(-i c, i \xi)=\sum_{r=0}^{\infty}(2 r+1) B_{2 r}^{0 n} \xi^{2 r} \text { for n even } \\
g_{\text {on }(-i c, i \xi)}^{\prime}=\sum_{r=0}^{\infty} 2 r B_{2 r}^{0 n} \xi^{2 r-1} \text { for nodd }
\end{gathered}
$$

Therefore the numerical means of calculating the radial functions are completely determined.

\subsection{Analytic Solutions}

The exact solution in (4.2) have been calculated in terms of the angle and radial oblate spheroidal functions. Although (4.2) is a short equation, writing a FORTRAN code for it has been challenging. There is a limited listing of the eigenvalues, coefficients, angle functions and radial functions in [11] and [12]. A portion of those values listed in [11] and [12] have been verified when debugging the code. In spite of the inadequate information, the code calculates satisfactorily. Figures 4.2 and 4.3 compare the analytic solutions obtained by the code and the corresponding BOR simulation results for thin disks. The exact solution in (4.2) involves a summation from zero to infinity for $n$, but a summation from zero to 30 proves to be adequate for the code. Comparing the analytic solution has proved that the BOR code is valid for a point source excitation as well. 
Total pressure field amplitude in the azimuthal plane

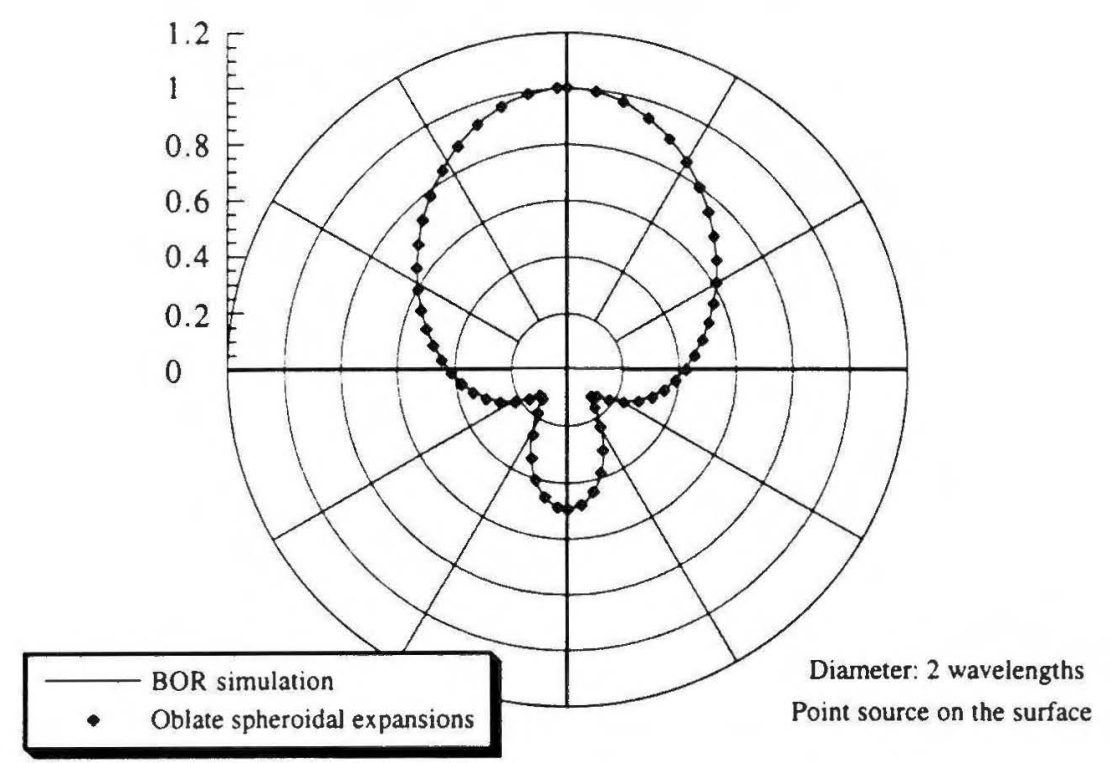

Figure 4.2 The BOR simulation and analytic diffraction solution of the disk 1

Total pressure field amplitude in the azimuthal plane

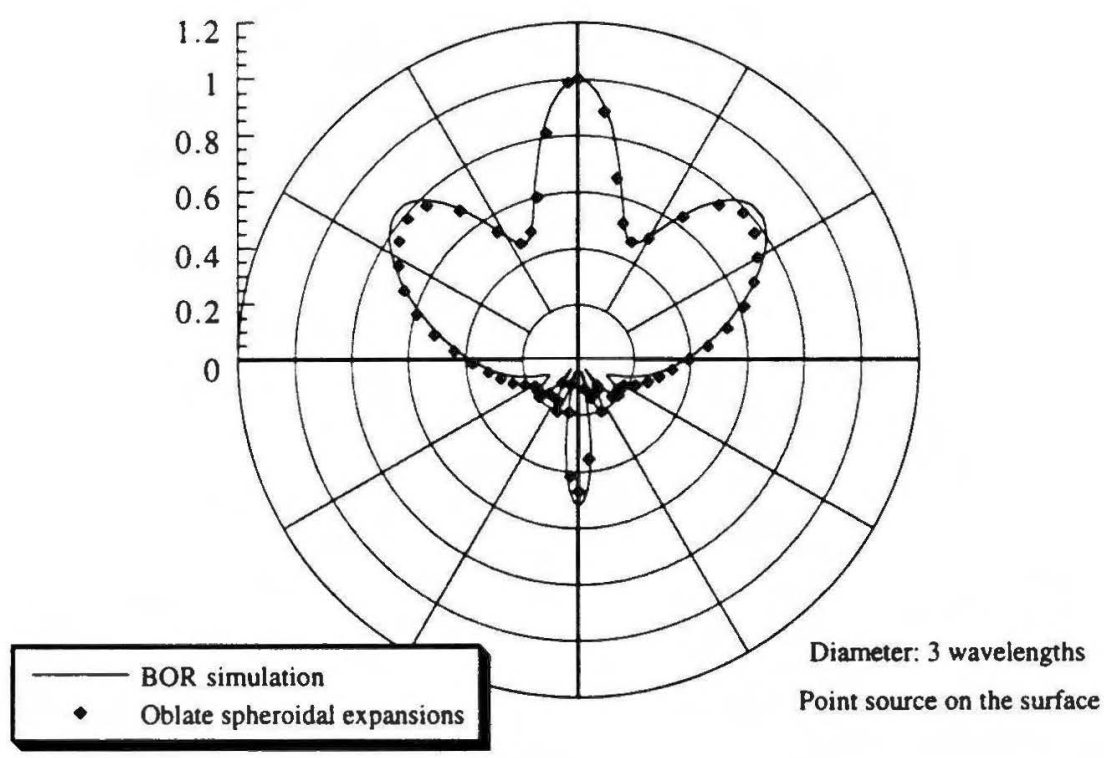

Figure 4.3 The BOR simulation and analytic diffraction solution of the disk 2 


\section{CHAPTER 5 CONCLUSIONS}

In this thesis numerical methods for calculating diffractions of acoustic waves have been investigated. Diffraction patterns have been accurately calculated using the MOM code for simple objects small compared to their wavelength. Chapter 2 introduces the MOM and presents the simulation results. The MOM code is most useful for studying diffractions of rectilinear objects, and it is not suitable for analyzing curved objects due to the unfriendly meshing scheme. The BOR code is written to compensate for the shortcomings of the MOM code. Diffraction patterns of axisymmetric objects with axisymmetric boundary conditions can be efficiently calculated by the BOR code. The diffraction patterns of spheres, parabolic reflectors, and thin disks have been successfully calculated, and the simulation results are shown in Chapter 3. Diffractions due to a plane wave incidence are easily compared with existing references. For a point source incidence, no graphical data are available, and only the analytic solution is expressed in terms of the spheroidal wave functions. In Chapter 4 the oblate spheroidal functions are discussed in detail. A code is written to calculate the analytic solution, and the calculation results have verified the validity of the BOR code for a point source incidence.

Note that the boundary conditions must also be axisymmetric for the BOR simulations. All of the BOR simulation results in this thesis are done in the full space. For a plane wave incidence, the direction of the propagation is parallel to the axis of symmetry, and for a point source incidence, the source is located on the axis. Analyzing objects in a half space, such as spheres above the flat ground, can also be done using the BOR code. Placing acoustic images of the original objects in the full space is equivalent to the original objects in the half space which loses the symmetry.

The BOR code can further be improved if it is modified to account for off-axis incidence. The current code assumes that there is no field variation on a plane perpendicular 
to the symmetric axis. For example, when the plane wave is incident at an angle from the axis of the symmetry, the incident fields on the surface of the object will vary accordingly. The scattered fields will have the same variation due to the variation of the incident fields. Finding out of a way to incorporate this variation in the BOR code will broaden its usage in analyzing the diffractions. 


\section{REFERENCES}

[1] C.A. Balanis, Advanced Engineering Electromagnetics, Wiley, New York, 1989.

[2] G.W. Swenson, Jr., E.R. Sandeen, L.L. Pater, and H.C. Zhuang, "The potential for mitigation of gun blast noise through sheltering of the source," Technical Report N-92/09, United States Army Corps of Engineers, Construction Engineering Research Laboratory, April, 1992.

[3] N. Morita, N. Kumagai, and J. R. Mautz, Integral Equation Methods For Electromagnetics, Artech House, Boston, 1990.

[4] J. M. Jin, The Finite Element Method In Electromagnetics, Wiley, New York, 1993.

[5] J. W. Benson, Y. L. Li, and G. W. Swenson, Jr., "A baffle-type directional microphone," J. Acoust. Soc. Am., vol. 95, pp. 2536-2538, 1994.

[6] A. F. Seybert, B. Soenarko, F.J. Rizzo, and D.J. Shippy, "A special integral equation formulation for acoustic radiation and scattering for axisymmetric bodies and boundary conditions," J. Acoust. Soc. Am. , vol. 80, pp. 1241-1247, 1986.

[7] J. J. Bowman, T.B.A. Senior, P.L.E. Uslenghi, Electromagnetic and Acoustic Scattering by Simple Shapes, Hemisphere Publishing Corp., New York, 1987.

[8]S. Wahlström, "The Parabolic Reflector as an Acoustic Amplifier," J. Audio Eng. Soc., vol. 33, pp. 418-429, 1985.

[9] A. Leitner, "Diffraction of Sound by a Circular Disk," J. Acoust. Soc. Am., vol. 21, pp. 331-334, 1949.

[10] F. M. Wiener, "The Diffraction of Sound by Rigid Disks and Rigid Square Plates," J. Acoust. Soc. Am., vol. 21, pp. 334-347, 1949.

[11] C. Flammer, Spheroidal Wave Functions, Stanford University Press, Stanford, 1957.

[12] M. Abramowitz and I. A. Stegun, Handbook Of Mathematical Functions With Formulas, Graphs, And Mathematical Tables, Dover Publications, Inc., New York, 1970. 


\section{USACERL DISTRIBUTION}

Chief of Engineers

ATTN: CEHEC-IM-LH (2)

ATTN: CEHEC-IM-LP (2)

ATTN: CEMP

ATTN: CEMP-CE

ATTN: CEMP-EA (2)

ATTN: CEMP-ZM

ATTN: CERD-L

ATTN: CERD-M (2)

ATTN: CERD-ZA

ACS(IM) 22060

ATTN: DAIM-FDP

CECPW 22310-3862

ATTN: CECPW-E

ATTN: CECPW-FT

ATTN: CECPW-ZC

Norwegian Defense

ATTN: Chief Test \& Dvlpmt Section

HQ USAREUR \& 7th Army

ATTN: AEAEN-EH

ATTN: Unit 29351

US Military Academy

ATTN: MAEN-A

ATTN: Civil Div Director

ATTN: Dept of Geo \& Env Engr

US Army Combat Sys Test Activity

ATTN: STECS-SO-R 21010-5059

Commander FORSCOM

ATTN: FCEN-RDF 30330-6000

CEWES 39180

ATTN: Library

CECRL 03755

ATTN: Library
US Army ARDEC 07806

ATTN: SMCAR-ISE

Engr Societies Library 10017

ATTN: Acquisitions

US Army Environmental Center

ATTN: SFIM-AEC-NR 21010

ATTN: SFIM-AEC-CR 64152

ATTN: SFIM-AEC-SR 30335-6801

ATTN: AFIM-AEC-WR 80022-2108

National Guard Bureau 20310

ATTN: NGB-ARI

US Military Academy 10996

ATTN: MAEN-A

ATTN: Facilities Engineer

Naval Facilities Engr Command

ATTN: Facilities Engr Command

Code 20YAZ (2)

US Army CHPPM

ATTN: MCHB-DC-EEN (5)

US Gov't Printing Office 20401

ATTN: Rec Sec;Deposit Sec (2)

Nat'1 Institute of Standards \& Tech

ATTN; Library 20899

Defense Construction Supply Center

ATTN: DCSC-WI 43216-500

Defense Tech Info Center 22060-6218

ATTN: DTIC-O (2)

$49+12$

$5 / 97$ 
DEPARTMENT OF THE ARMY

CONSTRUCTION ENGINEERING RESEARCH LABORATORIES

CORPS OF ENGINEERS

PO BOX 9005

CHAMPAIGN, ILLINOIS 61826-9005

BULK RATE

SOSTAGE

CHAMPAIGN IL

PERMIT NO. 871

OFFICIAL BUSINESS 